\title{
Understanding COVID-19 Pandemic: Molecular Mechanisms and Potential Therapeutic Strategies. An Evidence-Based Review
}

\author{
This article was published in the following Dove Press journal: \\ Journal of Inflammation Research
}

\begin{abstract}
Reem Hanna, (D) 1,2 Snehal Dalvi, (iD) ${ }^{1,3}$ Tudor Sălăgean, ${ }^{4}$ Ioana Delia Pop, ${ }^{4}$ loana Roxana Bordea, ${ }^{5} *$ Stefano Benedicenti ${ }^{1} *$

'Department of Surgical Sciences and Integrated Diagnostics, Laser Therapy Centre, University of Genoa, Genoa, Italy; ${ }^{2}$ Department of Oral Surgery, Dental Institute, King's College Hospital NHS Foundation Trust, London, UK; ${ }^{3}$ Department of Periodontology, Swargiya Dadasaheb Kalmegh Smruti Dental College and Hospital, Nagpur, India; 4ental College and Hospital, Nagpur, India; Department of Land Measurements and Exact
Sciences, University of Agricultural Sciences and Sciences, University of Agricultural Sciences and
Veterinary Medicine Cluj-Napoca, Cluj-Napoca, Romania; ${ }^{5}$ Department of Oral Rehabilitation, "Iuliu Hațieganu" University of Medicine and Pharmacy ClujNapoca, Cluj-Napoca, Romania

*These authors contributed equally to this work
\end{abstract}

\section{Video abstract}

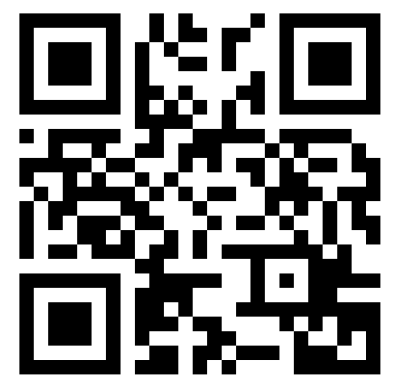

Point your SmartPhone at the code above. If you have a QR code reader the video abstract will appear. Or use: https://youtu.be/GI KmFyv03XU

Correspondence: Reem Hanna

Department of Surgical Sciences and Integrated Diagnostics, Laser Therapy Centre, University

of Genoa, Genoa, Italy

Tel +390103537446

Email reemhanna@hotmail.com

Tudor Sălăgean

Department of Land Measurements and Exact Sciences, University of Agricultural Sciences and Veterinary Medicine Cluj-Napoca, Cluj-Napoca, Romania

Tel +4074470737 I

Email tudor.salagean@usamvcluj.ro

\begin{abstract}
Initially, the SARS-CoV-2 virus was considered as a pneumonia virus; however, a series of peer reviewed medical papers published in the last eight months suggest that this virus attacks the brain, heart, intestine, nervous and vascular systems, as well the blood stream. Although many facts remain unknown, an objective appraisal of the current scientific literature addressing the latest progress on COVID-19 is required. The aim of the present study was to conduct a critical review of the literature, focusing on the current molecular structure of SARSCoV-2 and prospective treatment modalities of COVID-19. The main objectives were to collect, scrutinize and objectively evaluate the current scientific evidence-based information, as well to provide an updated overview of the topic that is ongoing. The authors underlined potential prospective therapies, including vaccine and phototherapy, as a monotherapy or combined with current treatment modalities. The authors concluded that this review has produced high quality evidence, which can be utilized by the clinical scientific community for future reference, as the knowledge and understanding of the SARS-CoV-2 virus are evolving, in terms of its epidemiological, pathogenicity, and clinical manifestations, which ultimately map the strategic path, towards an effective and safe treatment and production of a reliable and potent vaccine.
\end{abstract}

Keywords: SARS-CoV-2, COVID-19, virus pathogenicity, cytokines storm, diagnostic methods, immunotherapy, vaccine, antiviral, photobiomodulation therapy, PBMT, photodynamic therapy, PDT, clinical trials

\section{Summary}

1. The spread of SARS-CoV-2 virus has shown a potential zoonotic origin. Advancements in ongoing research activity is trying to identify further possible animal hosts of COVID-19

2. Classic Kawasaki disease (KD) has been identified in pediatric COVID-19.

3. Newer epidemiological facts, properties of the virus, immune responses against the virus and challenges in vaccine production are surfacing each day.

4. Prospective therapies: cellular therapy, ACE2 (vital component of renin-angiotensin system; RAS) treated with inhibitors of RAS; ACEI and AT1R.

5. Phototherapy can be considered as a potential treatment modality in COVID-19 management subjected to robust clinical trials.

\section{Introduction}

Since the World Health Organization (WHO) declared the severe acute respiratory syndrome coronavirus 2 (SARS-CoV-2) viral infection a pandemic, researchers and 
scientists have been making phenomenal efforts to understand the novel coronavirus behavior, clinical presentations, host-virus interaction and its impact on the host immune responses, as well as attempt to establish optimal treatments and produce a safe and effective vaccine. As of October 20th,2020, approximately 40,472,505 cases have been tested positive for coronavirus disease 2019 (COVID-19), including 1,119,283 deaths. ${ }^{1}$ Initially, SARS-CoV-2 was considered as a respiratory virus but a series of peer reviewed medical papers published in the last eightmonths, suggested that this virus attacks the brain, heart, intestine, vascular system via the blood stream. $^{2-4}$ The main driving force in violating the blood vessels endothelium lining is the spike protein (S), which binds to the angiotensin-converting enzyme 2 (ACE2), leading to a cytokine storm. ${ }^{5-8}$ In this context, many cases of multiple-organ dysfunction or failure have been reported in COVID-19 patients with comorbidities..$^{9-11}$ Nevertheless, many facts regarding the host-virus interaction still remain unknown. On this note, reliable diagnostic methods for an early virus detection and effective and safe treatment modalities to curb the spread of the disease have challenged clinicians and scholars across the globe. The current lines of COVID-19 patients management are palliative and restoring organ functionality. To date, more than 3652 studies are registered in clinical trials.gov and a large number of trials are ongoing, aiming to establish an effective treatment, as well a safe and potent vaccine, ${ }^{12}$ however this might take a length of time. Therefore, the quest for discovering alternative therapies shall persist until the ultimate cure, which is COVID-19 vaccine is produced.

This aim of this review article was to critically appraise the latest update on disease progression, by assimilating the current knowledge and understanding of COVID-19. The main objectives were to collect, scrutinize and objectively evaluate the available scientific evidence-based information and provide an overview of antiviral treatment modalities.

\section{Epidemiology Origin}

Although origins of SARS-CoV-2 are not entirely understood, the majority of patients in the initial stages of this outbreak reported its link to the Huanan South China Seafood Market, which is a live animal or "wet" market, suggesting a zoonotic origin of the virus. ${ }^{9,10}$ More recently, several studies that conducted genomic analyses suggesting that SARS$\mathrm{CoV}-2$ probably evolved from a strain found in horseshoe bats because the whole genome-wide nucleotide sequence of SARS-CoV-2 is $96 \%$ identical to SARS-like CoV isolated from an intermediate horseshoe bat (Rhinolophus affinis) $\mathrm{CoV}$, and $89 \%$ identical to two SARS-like CoVs isolated from Chinese horseshoe bats (Rhinolophus sinicus). ${ }^{13,14}$ Some researchers believe that the likelihood for the existence of an amplifying mammalian host intermediate between bats and humans remains questionable, due to the mutation in the original strain, which could have directly triggered virulence towards humans. ${ }^{15}$ Conflictingly, notable research has proven that the SARS-CoV-2 has been isolated from pangolins and $\mathrm{CoV}$ genomes found in the latter have approximately $85.5-92.4 \%$ similarity to SARS-CoV-2, suggesting that the pangolin may be a potential intermediate host for SARSCoV-2. ${ }^{16}$ There is also a substantial number of ongoing research to discover other probable animal hosts of SARS$\mathrm{CoV}-2$, which is of a great significance for the prevention and control of COVID-19. ${ }^{17}$

\section{Physicochemical Properties}

Most of the knowledge about the physicochemical properties of CoVs comes from SARS-CoV and MERS-CoV. ${ }^{17}$ The virus may survive and be detected on different surfaces for hours to days with a half-life ranging from $24 \mathrm{~h}$ on cardboard to approximately $72 \mathrm{~h}$ on plastic and stainless-steel surfaces, depending on the humidity and temperature. ${ }^{18,19}$ Like other $\mathrm{CoVs}$, it is sensitive to ultraviolet (UV) rays, heat $\left(56^{\circ} \mathrm{C}\right.$ for $30 \mathrm{~min}$ ) and most disinfectants. In order to achieve a $99.99 \%$ reduction of the virus, surfaces can be decontaminated by a one minute exposure to products such as, ether $(75 \%)$, ethanol (95\%), isopropyl alcohol (70-100\%), sodium hypochlorite $(0.21 \%)$, hydrogen peroxide $(0.5 \%)$, povidone-iodine (0.23-7.5\%), etc, except chlorhexidine gluconate. ${ }^{18,20}$ It has been found that soaps and detergents form a lather, which produce bubble-like structures called micelles that can degrade the fatty protective layer on the virus, thus deactivating and separating it from the surface of the skin as well as other surfaces, upon rinsing with water. ${ }^{18}$ Thus, they can be deemed as effective, if hand-washing procedure is performed correctly.

\section{Clinical Presentation and Disease Progression}

Evidence-based literature of COVID-19 clinical manifestations have shown that the stage of disease and severity of 
the symptoms and the corresponding care settings vary amongst the infected individuals (Figure 1). ${ }^{2,3,21-23}$ Most often, a suspected COVID-19 patient can exhibit symptoms such as; fever, cough, myalgia, dyspnea, fatigue, and altered sense of taste/smell. ${ }^{9,10}$ Approximately $15 \%$ of the infected patients have shown signs of fever, cough, and dyspnea, ${ }^{10}$ whilst the less common symptoms were as follows: sputum production, sore throat, gastrointestinal symptoms, chest pain, confusion, dizziness, headache, rhinorrhoea or nasal congestion, hemoptysis, conjunctivitis, cutaneous manifestations, and anorexia. ${ }^{10}$ Approximately $90 \%$ of the total infected population has exhibited more than one symptom. ${ }^{10}$ Many studies have been conducted to identify the disease progression roadmap., ${ }^{2,3}$ The results of the findings were based on the onset of the disease and immediately after contracting the virus, which revealed that every infected individual is in an asymptomatic state. ${ }^{2}$ This phase is known as viremia, which lasts for approximately 1-2 days. ${ }^{2,3}$ Although the localized spread of the infection, corresponding with limited innate immune response occurs at this stage and the viral burden may be low, patients are infectious and the virus can be detected via nasal swabs. ${ }^{2,3}$ Subsequently, over the next few days the virus replicates and spreads through the respiratory tract along the air conduction pathway, initiating a further stronger immune response. ${ }^{2,4}$ The positive results of nasopharyngeal swabs and early immune markers reveal a presence of SARS-CoV-2, during which the clinical symptoms are predominant at this stage. Alternatively, the acute phase in this context is termed as the pneumonia phase, ${ }^{3}$ in which patients are home-quarantined and most often are asked to self-isolate and continuously monitored by health officials and or placed on symptomatic therapy. If the host immune response is effective, almost $80 \%$ of the infected patients remain mildly infected. ${ }^{4}$ In this case, the virus is effectively suppressed and the disease is contained in the upper conducting airways and subsequently, the patient enters a recovery phase. ${ }^{3}$ Unfortunately, the remaining $20 \%$ of the population have comorbidities and are considered as immunosuppressed, ${ }^{4}$ where the disease progresses to an advanced stage. On this note, a severe form of the disease develops with a formation of "ground glass" pulmonary infiltrates detected on chest computed tomography (CT), associated with hypoxia and successively the disease deteriorates to an acute distress respiratory syndrome (ARDS) and multi-organ failure (Figure 1)., ${ }^{2,4}$ Approximately $2.3 \%$ of the reported cases resulted in fatal outcomes. ${ }^{4}$ It has been noted that the severely affected COVID-19 patients are more contagious than the mildly affected counterparts, although asymptomatic infected persons in an incubation period have the capability to spread the infection. ${ }^{2,3}$ Apart from the abovementioned scenarios, atypical clinical features involving delayed presentation of symptoms have also been reported among older individuals as well as those with medical comorbidities. $^{22}$ At present, although several cross-sectional studies have reported a high prevalence of asymptomatic infection, as of now, since asymptomatic patients are

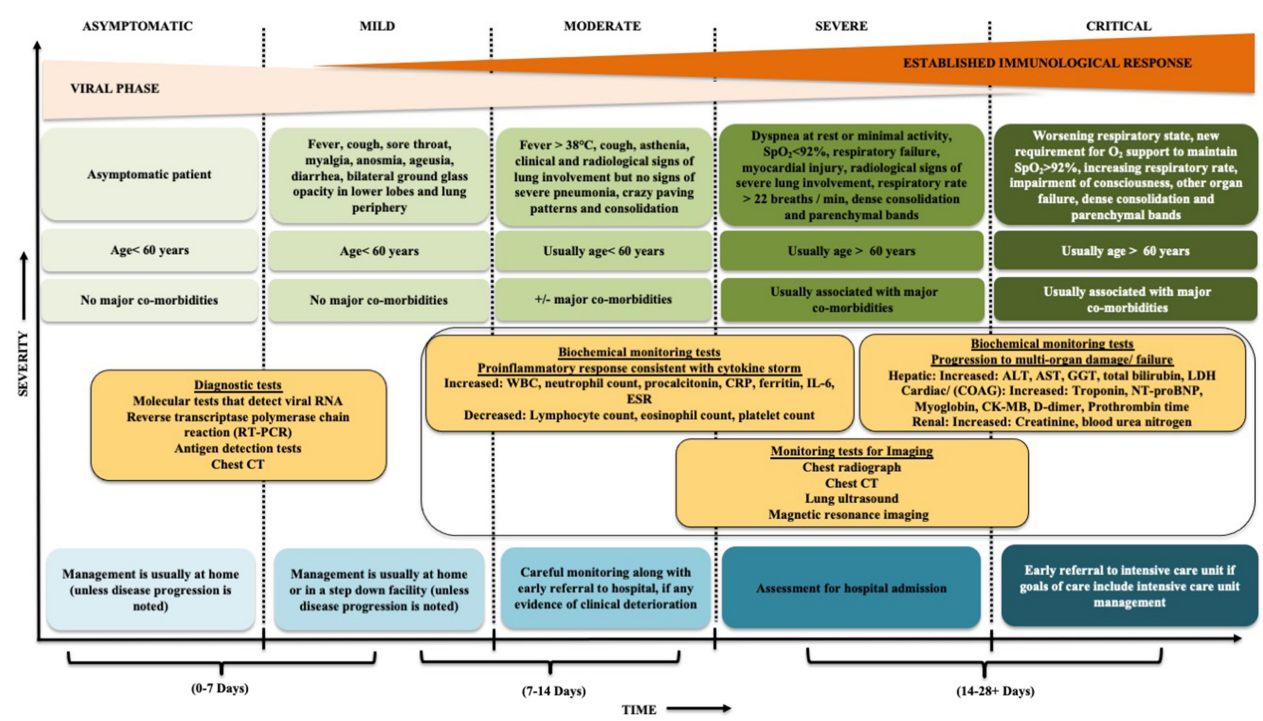

Figure I Modified schematic diagram illustrates COVID-I9 clinical presentation, diagnostic investigations, assessment of disease severity and consideration of care settings accordingly. $2,3,21-23$ 
not routinely tested, the ratio of asymptomatic to symptomatic infection is uncertain and needs to be explored further. $^{22}$

\section{Susceptible Population}

Notable research has pointed out that all populations are generally susceptible to SARS-CoV-2. It is factual that elderly patients with or without underlying comorbiditiescardiovascular diseases (CVDs), diabetes mellitus (DM), obesity- have a low immune function and response $\mathrm{e}^{9-11}$ and are at a high risk, due to a failure to repair the damaged epithelium and reduced mucociliary clearance, leading to a rapid migration of the virus to the lung gas exchange units and further across multiple organs. ${ }^{24}$ Reports presented by the Center for Disease Control (CDC) China states that the population, within the age range of 30-79-year-olds, were the most severely affected. ${ }^{25}$ This data is in accordance to the global reports. ${ }^{11,26}$ It has also been shown that men have a higher rate of disease severity and mortality rate, independent of age and susceptibility, compared to women.11,26,27 In addition, pregnant women and new-born babies infected with SARS-CoV-2 are also prone to develop a severe pneumonia. ${ }^{28}$ Thus, the treatment of these vulnerable patients should be given prime importance. Until recently, no severe cases had been reported among young children and it was assumed that a robust innate immune response is a significant factor for disease outcome. ${ }^{9}$ However, surprising new evidence, linking COVID-19 with Kawasaki disease (KD) has been reported. ${ }^{29}$ Jones et al, reported a case of a six-month-old infant diagnosed with classic KD who also screened positive for COVID-19 has showed signs of fever and mild respiratory distress. ${ }^{29}$ The association of KD with COVID-19 is more than a mere coincidence because reports of the former's correlation with other human CoVs have been established in the past. ${ }^{30} \mathrm{In}$ this context, further evidence is importantly required to achieve a definitive line of action of pediatric COVID-19 management. An association between the number of infected patients with ethnic origin, race, socioeconomic status and illiteracy have also been acknowledge on a global platform. ${ }^{11,26,27}$ The CDC has stated that people living in rural communities, experiencing homelessness, newly settled refugee populations, people residing in nursing homes/long-term health-care facilities/group homes for the disabled have been shown to be at a high risk of acquiring COVID-19 infection and hence should take extra precautions. ${ }^{31}$ Recent evidence highlighted that the Bacille Calmette-Guérin (BCG) vaccine could have a potential role in reducing the viremia caused by SARS-CoV-2 and the subsequent illness severity. ${ }^{32}$ However, the research in this context is ongoing and conclusive results can be expected only in the near future.

\section{Transmission Routes (Disease Spectrum)}

The primary route of contagion is through close contact with infected individuals, which is often exhibited via respiratory droplets, arising from coughing, sneezing, or talking. ${ }^{33}$ However, this may not be the only route of transmission. ${ }^{33}$ Close contact has also been confirmed to be a potential source for spread of SARS-CoV-2. ${ }^{34}$ Touching contaminated surfaces and then the face also could increase the likelihood of the disease spread. This occurs through a direct or an indirect contact with the mucous membrane of the eyes, mouth and nose. ${ }^{34}$ In addition, recent evidence has indicated that the airborne transmission route associated with an aerosol generation is the cause of the disease. ${ }^{34}$ Aerosol generation in an enclosed environment such as a dental clinic can result in continuous exposure to a high concentration of aerosols containing the virus. It has revealed that SARS-CoV-2 remains viable for approximately three hours in aerosols. ${ }^{24}$

\section{Incubation Period}

The mean incubation period of SARS-CoV-2 is three to seven days (range: 2-14 days), which represents the current official estimated range for the novel coronavirus COVID-19. ${ }^{35}$ This finding is in accordance to the incubation periods reported by the WHO (two and 10 days), ${ }^{36}$ China's National Health Commission (NHC) (10-14 days) ${ }^{37}$ and the United States' CDC (two and 14 days). ${ }^{38}$ According to the pooled analysis of confirmed COVID-19 cases reported between January 4 and February 24, 2020, as an incubation period of five days has been suggested by the research group at the Johns Hopkins University, USA. $^{35}$ These authors suggested that the latent period of SARS-CoV-2 is consistent with those of SARS-CoV (mean five days, range: 2-14 days), which coincided with the Canadian research group's findings ${ }^{39}$ and with MERS-CoV (mean 5.7 days, range: $2-14$ days). ${ }^{39}$ In spite of the long transmission period of the virus, evidence suggests that asymptomatic COVID-19 patients can effectively transmit infection during the incubation periods. ${ }^{40}$ Controversially, some discrete cases have been reported with a very longer incubation period of 24 days, ${ }^{41} 27$ days, ${ }^{42}$ and 19 days. $^{43}$ According to the WHO, the long incubation period may suggest a possibility of a double 
exposure and a potential for relapse, which needs further investigations. $^{36}$

\section{Histopathological Characteristics}

The study of the pathological characteristics of a severe case of COVID-19 can assist in designing a treatment strategy for the acute and severely ill patients, which ultimately reduces the mortality rate. ${ }^{21}$ Nonetheless, the histopathological examination of the lung tissue specimen obtained from a SARS-CoV-2 infected patient shows a desquamation of pneumocytes along with the formation of hyaline membrane and interstitial mononuclear inflammatory infiltration, indicating ARDS. ${ }^{44}$ Additionally, the intraalveolar spaces consisted of multinucleated giant cells, which were indicative of suggesting viral cytopathic-like changes ${ }^{44}$ Studies have confirmed similarity in the pathological findings of COVID-19 with SARS and MERS. ${ }^{45}$ Deshmukh et al, and co-workers have recently conducted a systematic review to summarize the histopathological observations in COVID-19. The authors have presented a critical appraisal of 45 studies which have assessed the latest histopathological changes in different organs observed after autopsy of COVID-19 cases. They concluded that although the respiratory and immune systems are the worst affected, other systems such as, cardiovascular, urinary, gastrointestinal tract, reproductive system, nervous system, and integumentary system also show significant histopathological changes which are especially observed in elderly cases and those with comorbidities. ${ }^{46}$

\section{Diagnostic Tests and Radiographic Imaging Characteristics (Chest CT Presentation)}

A COVID-19 patient can show the following changes on chest CT scan: bilateral pulmonary parenchymal groundglass opacity, pulmonary consolidation and nodules, bilateral diffuse distribution, sometimes with a rounded morphology and a peripheral lung distribution. ${ }^{47}$ During an early phase of the disease, multiple small patchy shadows and interstitial changes are evident in the lung periphery and appreciated on a chest CT image. ${ }^{16}$ As the severity of the disease progresses, changes can be observed in the bronchi, which gradually manifest across the entire lung with infrequent interlobar pleural thickening and pleural effusion..$^{21,47}$ On this note, the moderate lung abnormalities such as; patchy groundglass opacities' (normalized during treatment) findings observed in the adults' CT scan been reported in a study evaluated CT changes in children. ${ }^{48}$

The rapid and precise detection of the SARS-CoV-2 is quintessential to win this race against time. This has been facilitated through the laboratory diagnosis, using RTqPCR technique, which is based on detection of nucleic acid sequences. ${ }^{21}$ RT-qPCR or viral gene sequencing of nasopharyngeal and oropharyngeal swabs (stool, sputum, or blood samples) have been conducted to facilitate SARSCoV-2 detection. ${ }^{49}$ However, this testing method has several shortcomings such as, difficulty during sample collection, need for close contact with health professionals, which can increase the risk of contagion, initiation of bleeding, and gag reflex. ${ }^{21,50}$ Furthermore, evidence has shown that COVID-19 detection varies from different sample sites, ${ }^{51}$ in which nasopharyngeal swabs are more reliable than oropharyngeal swabs. ${ }^{52}$ In spite of the controversies growing around this method, there has been a worldwide exponential ramping up of testing procedures over the last eight months. The detection of the virus in saliva has also been proven, suggesting that saliva might serve as, a noninvasive site, to diagnose and monitor the rate of infectivity. ${ }^{53}$ Nonetheless, coronavirus swab test can only detect an ongoing viral status and provides no information, if the individual was previously infected and recovered. Moreover, the results of the swab tests take a minimum of $24 \mathrm{~h}$ for diagnosis. ${ }^{54}$ In contrast to these findings, the use of a serological test (antibody testing) can identify if an individual was previously infected with COVID-19 and recovered. ${ }^{54}$ This involves a finger-prick testing method with results obtained at approximately 15-30 mins, at a higher level of diagnostic accuracy has been reported than the swab test. ${ }^{54}$ Research on the use of synthetically produced RNA fragments of the SARS-CoV2 , as a diagnostic tool has shown notable progress and this could hold a promising role in the future. ${ }^{55}$ Figure 1 provides an illustration of the diagnostic tests as well as various biochemical and imagining tests that are performed for monitoring COVID-19 patients.

\section{Pathophysiology and Etiopathogenesis \\ General Characteristic Features and Morphology of SARS-CoV-2}

The Coronaviridae family (subfamily: Orthocoronaviridae, order: Nideovirales), are a large family of enveloped, nonsegmented, positive-sense, single-stranded RNA 
viruses as observed under an electron microscope. ${ }^{15}$ This family of viruses are genotypically and serotypically further subclassified as; alpha-coronavirus $(\alpha-\mathrm{CoV})$, betacoronavirus $(\beta-\mathrm{CoV})$, delta-coronavirus $(\delta-\mathrm{CoV})$, and gamma-coronavirus $(\gamma-\mathrm{CoV}) .{ }^{15,17}$ Several systemic ailments such as, respiratory, enteric, hepatic, and neurological diseases in different animal species can be caused by this family of viruses. ${ }^{15}$ Human $\mathrm{CoV}$ infections are specifically caused by $\alpha$ - and $\beta$-CoVs. ${ }^{5}$ SARS-CoV-2 belongs to the subdivision $\beta-\mathrm{CoV}$, which is similar to its predecessors, severe acute respiratory syndrome-coronavirus (SARS$\mathrm{CoV}$ ) and Middle East respiratory syndrome (MERS$\mathrm{CoV}) .{ }^{15}$ SARS-CoV-2 is round or elliptic and often has a pleomorphic form, and a diameter of approximately $60-140 \mathrm{~nm} .{ }^{15}$ The genome that has a size of approximately 30 kilobytes (kb) contains 29,891 nucleotides, encoding for 9860 amino acids. ${ }^{15}$ Of the several proteins possessed by the virus, there are four structural proteins, which consist of the spike surface glycoprotein, the membrane protein, the envelope protein and the nucleocapsid protein, which are fundamental for the assembly and potential for infectivity, of SARS-CoV-2. ${ }^{21}$

\section{Structure and Function of the Spike Protein}

The CoVs derive their peculiar name from the Latin term "coronam", which means crown, due to the presence of spike surface glycoproteins on the envelope. ${ }^{15}$ The host viral interaction takes place in five steps namely, attachment, penetration, biosynthesis, maturation, and release. ${ }^{56}$ The spike protein plays a crucial role in facilitating the virus-host cell attachment. Upon attachment to the host receptors, the virus penetrates via endocytosis or membrane fusion into the host cells. ${ }^{56}$ Host proteases have the potential to cleave the spike protein into an $\mathrm{N}$-terminal S1 region for primary attachment and a membrane-bound C-terminal S2 region for viral infusion. ${ }^{57}$ In order to engage a host receptor, the receptor-binding domain (RBD) of the S1 subunit undergoes hinge-like conformational movements, which transiently hides or exposes the determinants of receptor binding. ${ }^{5,6}$ These two states of the S1 subunit can be regarded, as the down conformation (inaccessible state of the receptor) and the up conformation (accessible state of the receptor).${ }^{5,7}$ Upon binding of the S1 region of the protein to the host receptor cells, the pre-fusion trimer is destabilized, leading to shedding of the S1 subunit. ${ }^{8}$ This facilitates the transition of the S2 sub-unit into a highly stable postfusion conformation. ${ }^{8}$ Subsequent to the transfer of all the viral contents into the host cells, the viral RNA enters the nucleus for replication and to biosynthesize the viral proteins. The newly formed viral particles then undergo maturation and release phases. ${ }^{56}$ Researchers are optimistic that understanding the structure and function of the spike protein can aid in the development of various antiviral drug regimens, as well in a direct development of vaccines in the near future. ${ }^{57}$

\section{Host Defense Against SARS-CoV-2 Viral Interaction}

Initially, the SARS-CoV-2 targets the nasal and bronchial epithelial cells, alveolar epithelial type I and II pneumocytes and capillary endothelial cells through the viral structural spike (S) protein to initiate an inflammatory response, by activating the inflammatory pathways of the immune system. ${ }^{58,59}$ Scientific evidence of the spike protein binding to angiotensin converting enzyme 2 (ACE2), which is present in the host cell has identified the latter as a functional receptor for SARS-CoV. ${ }^{60}$ The ACE2 receptor is predominantly expressed in the lung epithelial cells, heart, ileum, kidney and bladder. ${ }^{61}$ The entry of the SARS-CoV-2 into the host cells is promoted by the cleaving of ACE2 by the type 2 transmembrane serine protease (TMPRSS2), which is present in the host cell, particularly the alveolar epithelial type II pneumocyte cells. ${ }^{61-63}$ The inflammatory response, consisting both of the innate and the adaptive immune response (comprising of humoral and cell-mediated immunity) can impair lymphopoiesis and increase the $\mathrm{T}$ lymphocyte cell apoptosis resulting in profound lymphopenia. ${ }^{64-66}$

Cytokines are an essential part of the inflammatory process and are produced by several immune cells including the innate macrophages, dendritic cells, natural killer cells and the adaptive $\mathrm{T}$ and $\mathrm{B}$ lymphocytes. ${ }^{67}$ As the viral load increases, an exaggerated and unusual hostimmune response comes into play, leading to a continuous activation and expansion of immune cells, lymphocytes, and macrophages, which produce immense amounts of cytokines, resulting in a cytokine storm (CS), which is a serious life-threatening condition. ${ }^{67}$ The clinical findings associated with CS are linked to the action of the proinflammatory and anti-inflammatory cytokines such as, Interleukin-1 (IL-1), IL-6, IL-18, IL-10, IFN- $\gamma$, and tumor necrosis factor-alpha (TNF- $\alpha$ ), which are elevated in the serum of COVID-19 patients. ${ }^{68}$ IL- 6 and TNF- $\alpha$ have been recognized to play a major role 
sequence of events occurring, as a result of the CS and consequent disease progression. ${ }^{67}$

In later stages of the infection when the viral replication is accelerated, an exponential rise in cytokine levels result in the influx of other immune cells such as; macrophages, monocytes, neutrophils, and $\mathrm{T}$ cells from the circulation into the site of infection. ${ }^{67,68}$ Various destructive events start to occur which are as follows: destabilization of the epithelial-endothelial barrier, damage of vascular barrier, capillary damage, infection spreading to the pulmonary capillary endothelial cells, diffuse alveolar damage, diffuse thickening of the alveolar wall with mononuclear cells and macrophages infiltrating airspaces appearing as ground-glass opacities on CT imaging and pulmonary edema filling the alveolar spaces with formation of hyaline membrane, dysfunctional alveolar-capillary oxygen transmission, and impaired oxygen diffusion capacity (Figure 2). ${ }^{44,64}$ Lung injury is one consequence of the cytokine storm that can progress into early phase acute respiratory distress syndrome (ARDS) or its more severe form. ${ }^{64,67,68}$

With a further progression into COVID-19 disease severity, a disproportionate activation of the complement system stimulates the coagulation cascade, endothelial cells and platelets, inhibits fibrinolysis and results in the formation of microthrombi and a high incidence of thrombotic complications in critically ill patients. ${ }^{69,70}$ The advancement of the disease process causes dysregulated host immune response mediated viral sepsis (critical organ dysfunction) which may further contribute to multi-organ failure. ${ }^{64}$ The characteristic clinical presentation of CS includes; signs of overwhelming systemic inflammation, hyperferritinemia and hemodynamic instability. Additionally, researchers have successfully linked the excessive production of pro-inflammatory cytokines as one of the major contributing factors in the development of ARDS in COVID-19 patients. ${ }^{9,10,71}$ ARDS leading to low oxygen saturation levels is a major cause of multi-organ failure and mortality in COVID-19. ${ }^{67}$ Hence, the early recognition of CS and the prompt treatment can improve the clinical outcome of the COVID-19 infection. ${ }^{67,72}$ Several antiviral and anti-inflammatory drug therapies have been proposed for treating SARS-CoV-2 mediated CS, in order to decrease both the morbidity and mortality in COVID-19 patients, and a comprehensive review and critical appraisal of these therapies has been made in this review.

\section{Current Scenario for Treatment of COVID-I 9}

\section{General Management}

Presently, there are no definitive therapeutic protocols for COVID-19 management. ${ }^{21}$ For suspected and mild confirmed cases, isolation in designated areas or self-isolation at home has been suggested. ${ }^{21,73}$ The general line of treatment revolves around establishing symptomatic relief includes: ample bed rest, maintaining a good water-electrolyte balance and monitoring vital signs (temperature, heart rate, oxygen saturation, blood pressure, pulse rate, respiratory rate). ${ }^{21,73}$ Apart from these, it is important to note that all patients irrespective of the disease severity

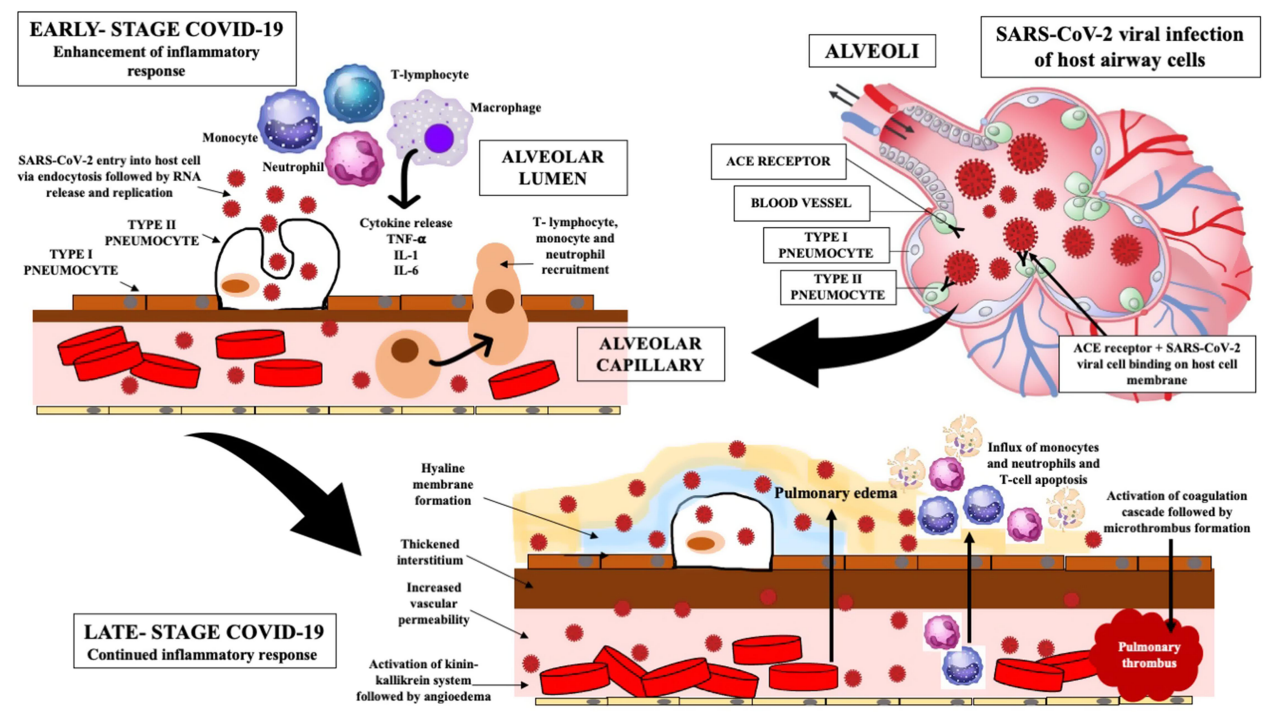

Figure 2 Modified schematic diagram illustrates the etiopathogenesis and pathophysiology of COVID-19. Data from Wiersinga et al. ${ }^{64}$ 
need to receive a balanced diet full of high nutritive value with a good intake of foods rich in antioxidants. ${ }^{73}$ An overview of the contemporary trends for the treatment COVID-19 has been essayed in this review.

\section{Antiviral Therapy}

Although specific antiviral drugs and drug regimes have not been established to date, a variety of antiviral therapeutic drugs have been tried and tested worldwide with fluctuating results, to obtain COVID-19 symptomatic relief. ${ }^{17}$ The use of these drugs has been reported through many case series and case reports. On this note, the level of usage of these drugs is low and controversial, due to the limited number of human clinical trials. ${ }^{17,74}$ The advantages and drawbacks of the potential antiviral therapeutic drugs that have been utilized for COVID-19 management are listed in Table 1 and a detailed review is been presented below:

\section{Antiviral Cytokines}

Antiviral cytokines are proteins, having broad antiviral activity which exhibits both direct inhibitory effects on viral replication and spread through several mechanisms and supporting an immune response to clear viral infection which will enhance the adaptive immunity and improve the host resistance to the viral infection. ${ }^{75}$ In previous supporting literature, the use of interferons alpha and beta (INF $\alpha$ and INF $\beta$ ) to mitigate SARS-CoV and MERS-CoV infections have been proven, ${ }^{75,76}$ as well in combination with other antiviral drugs such as, lopinavir/ ritonavir, ribavirin, remdesivir, corticosteroids have been reported. ${ }^{77-80}$ INF $\beta$ is better tolerated than INF $\alpha$ and no signs of adverse birth outcome have been noted with their use in COVID-19 management. Some frequently encountered disadvantages with the utilization of antiviral cytokine therapy include, flu-like symptoms, nausea, fatigue, weight loss, hematological toxicities, elevated transaminases, and psychiatric problems (Table 1). ${ }^{81}$ The potential for added toxicity with concurrent use of other immunomodulators and chemotherapeutic agents is seen as the most serious drug-drug interactions with this therapy.

In an uncontrolled, exploratory study by Zhou et al, 77 adults who had tested positive for COVID-19 were treated with either nebulized IFN- $\alpha 2 b$ (five milli international units twice a day), or arbidol (200 $\mathrm{mg}$ three times a day; tds) or a combination of IFN $\alpha 2 b$ and arbidol. The authors concluded that the patients who received IFN $\alpha 2 b$ with or without arbidol showed a significant reduction in the detectable virus duration in the upper respiratory tract along with a reduction in the duration of elevated blood levels for IL-6 and CRP. ${ }^{81}$ However, as per the recommendations of the COVID-19 Treatment Guidelines Panel, National Institutes of Health (NIH), the use of interferons for severe of critical COVID-19 illness must be avoided except in a clinical trial. The panel has provided a substantial evidence to highlight the drawbacks of interferons in the previous coronavirus infections, as well underlying toxicities that prevail over the potential benefits of this treatment strategy. ${ }^{82}$ Concurrently, the NIH are conducting a study called the Adaptive COVID-19 Treatment Trial 3 (ACTT 3), which would take place in the USA and other sites globally. The combination of INF $1 \beta$ and remdesivir for treating COVID-19 has been evaluated in a few smallscale randomized controlled trials (RCTs) and hence the current trial has been undertaken on a larger platform. ${ }^{83}$ The various clinical trials related to the utilization of this therapy in COVID-19 patients are listed in Table $2 .^{83-85}$

\section{Antiviral Drugs}

The nucleoside analogs are vital antiviral drugs, which have been used most commonly to treat HIV, hepatitis B virus (HBV), cytomegalovirus (CMV), HSV infections. ${ }^{86}$ These drugs impair viral replication by competitive inhibition of the viral polymerase or termination of the DNA chain. ${ }^{86}$ Due to its wide use in the past during the SARS pandemic, ribavirin was one of the first drugs tested as a potential remedy for COVID-19. ${ }^{87}$ In the past, it has been utilized in combination with steroidal drugs, as well as IFN $\beta{ }^{88}$ Owing to an existing inventory and a reliable supply chain, it can be used globally for COVID-19 management. However, hepatic injury, myopathy, neuropathy, bone marrow suppression, and pancreatitis are some of the most common adverse effects that have been encountered in the use of this drug (Table 1). ${ }^{86,89}$ Another important nucleoside analog, remdesivir was utilized on a large-scale for the treatment of SARS-CoV and MERS-CoV. ${ }^{90}$ The antiviral efficacy of the drug against SARS-CoV-2 has been proven through in vitro analysis. ${ }^{91}$ In fact, this drug was also utilized in the treatment of COVID-19 in the USA. $^{92}$ Although, nucleoside analogs have been used extensively to treat viral pneumonia, these drugs have several adverse effects such as, diarrhea, rash, renal failure, and hypotension, increased liver enzymes (Table 1). ${ }^{93}$ In a recent randomized double-blind, placebo-controlled, multicenter trial at 10 hospitals in Hubei, China, the antiviral efficacy of remdesivir was tested on COVID-19 


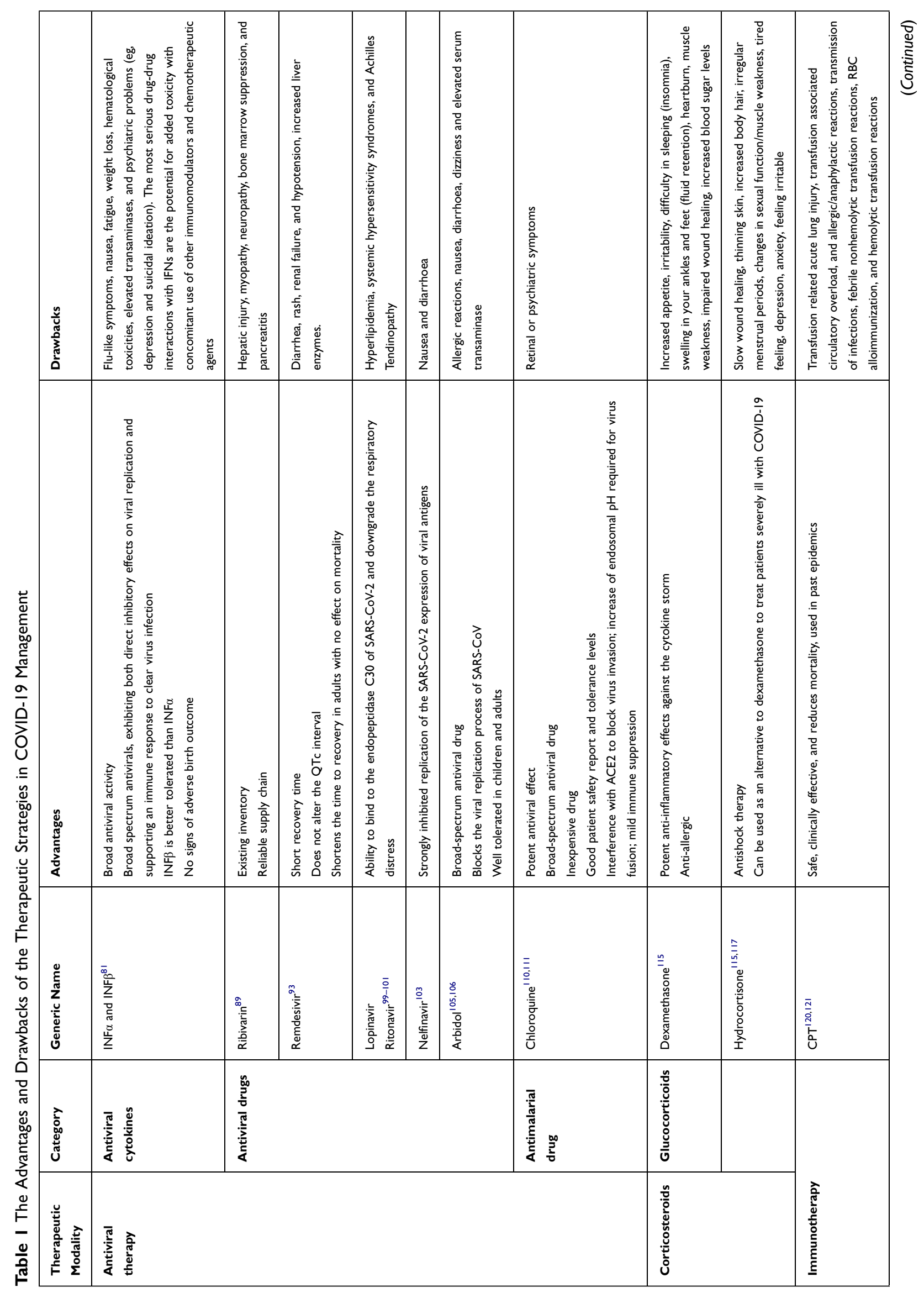




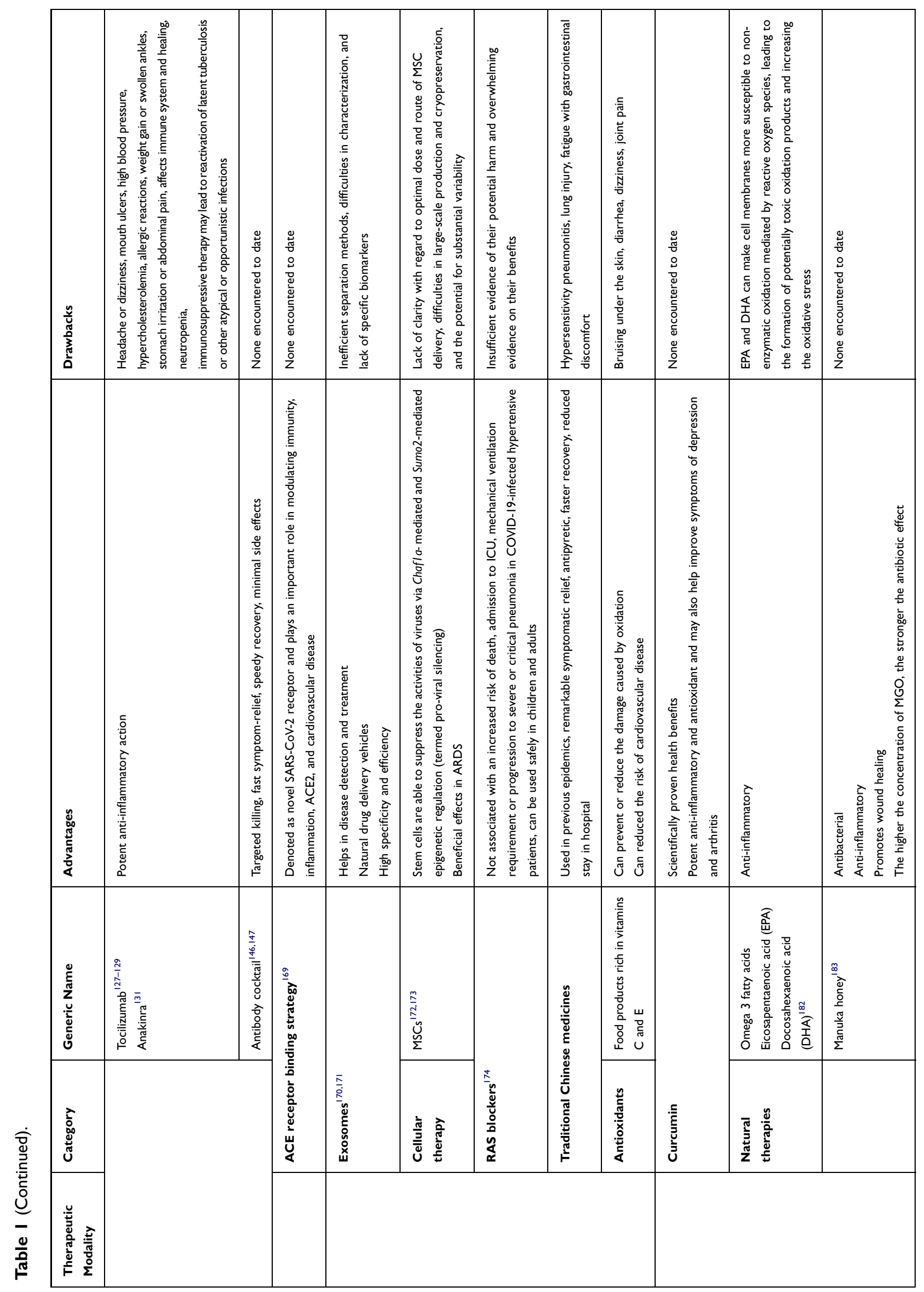




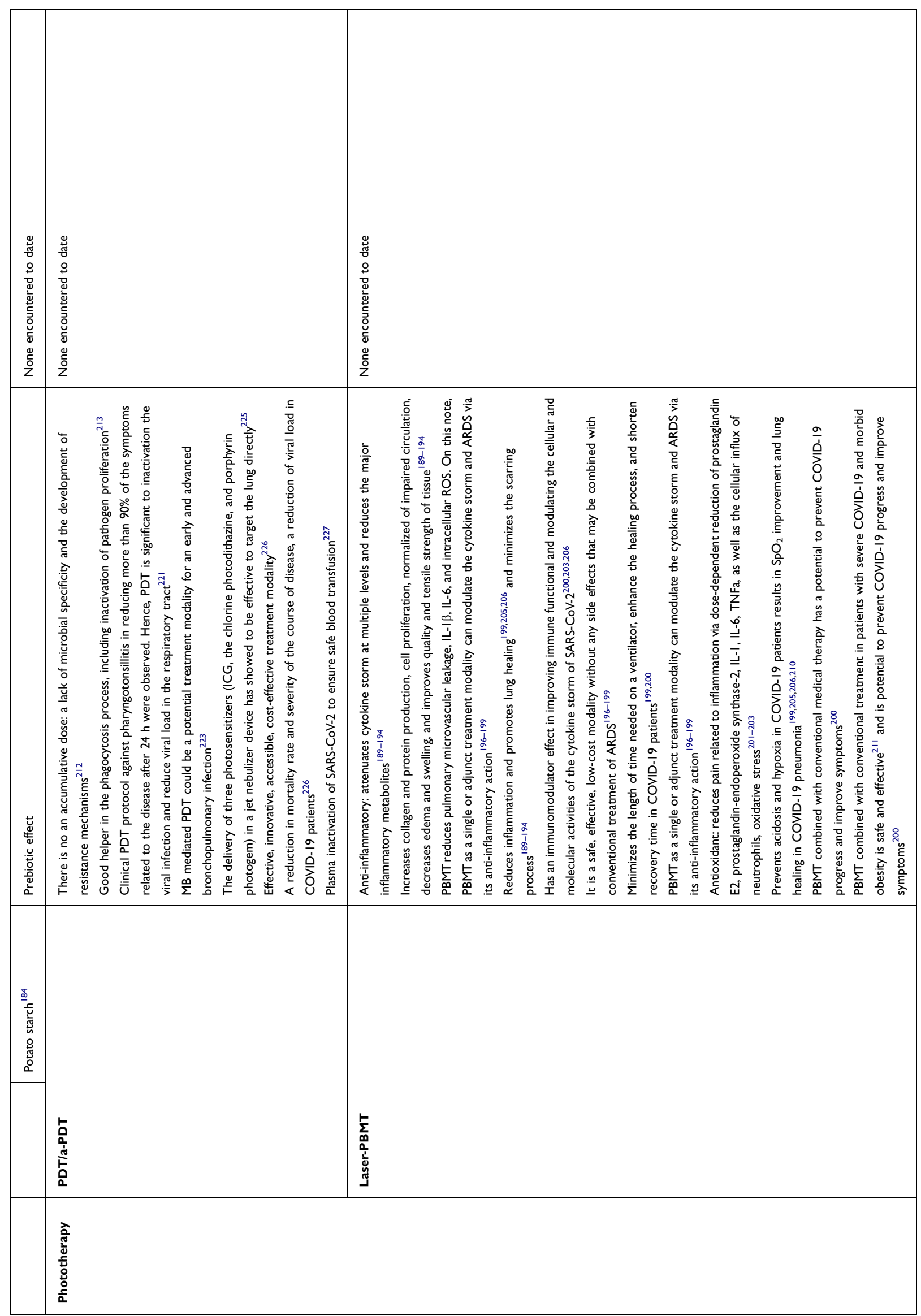




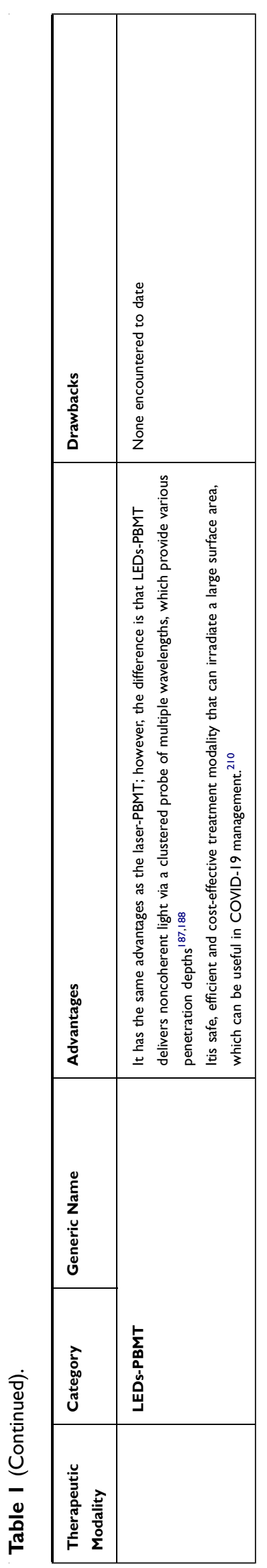

patients. Over a period of 10 days, the patients were randomly assigned in a 2:1 ratio to intravenous remdesivir. The dosage was $200 \mathrm{mg}$ on day one, followed by $100 \mathrm{mg}$ on days $2-10$ in single daily infusion) or the same volume of placebo infusions. Although the findings were not statistically significant, the test group patients showed a faster clinical improvement than the placebo group. ${ }^{94}$ To date, several clinical trials have been registered to assess the efficacy of these nucleoside analogs in COVID-19 management which are listed in Table $2 .^{83,95-98}$

The HIV protease inhibitors are a class of antiviral drugs, which play a vital role in the highly active antiretroviral therapy (HAART) utilized in HIV/AIDS management. ${ }^{99}$ Lopinavir and ritonavir, apart from their virtually identical molecular structures, inhibit the HIV protease by impairing cytochrome P450 activity, which affects the viral replication and synthesis (Table 1). ${ }^{99}$ Therapeutic efficacy of these drugs has been reported in the management of SARS and MERS. ${ }^{100}$ It has been suggested that these drugs have the ability to bind to the endopeptidase $\mathrm{C} 30$ of SARS-CoV-2 and downgrade the respiratory distress. ${ }^{101}$ However, higher doses of both lopinavir and ritonavir have been linked to a number of toxicity conditions such as, hyperlipidemia, systemic hypersensitivity syndromes, and Achilles tendinopathy. ${ }^{101}$ Hence, the safe use of these drugs is of utmost importance and should be performed only in the light of substantial evidence. Nonetheless, a recent review paper by Khalili et al, indicates that there are a number of ongoing clinical trials in which many of them, being conducted in China are to evaluate the role of ribavirin in the COVID-19 outbreak. ${ }^{89}$ Some of these studies have compared the effectiveness of ribavirin vs many of the antiviral drugs mentioned above, whilst the others have been designed to evaluate whether these drugs are more effective when used in different combinations. ${ }^{61}$ The results of these studies will indeed show researchers the path forward. Nelfinavir is a selective HIV protease inhibitor, whose antiviral efficacy has been proven against SARS-CoV. ${ }^{102}$ This drug can strongly inhibit the replication of the SARS-Cov-2 expression of viral antigens (Table 1). ${ }^{103}$ The potential implications for the use of this drug in COVID-19 patients, is nevertheless, subject to the development of the scientific evidence. The most common side effects of this drug are nausea and diarrhea (Table 1). ${ }^{100}$ The clinical trials utilizing this class of antiviral drug are listed in Table 2. ${ }^{85,104}$

Alternatively, the use of arbidol, as a broad-spectrum antiviral compound blocks the viral replication process of 







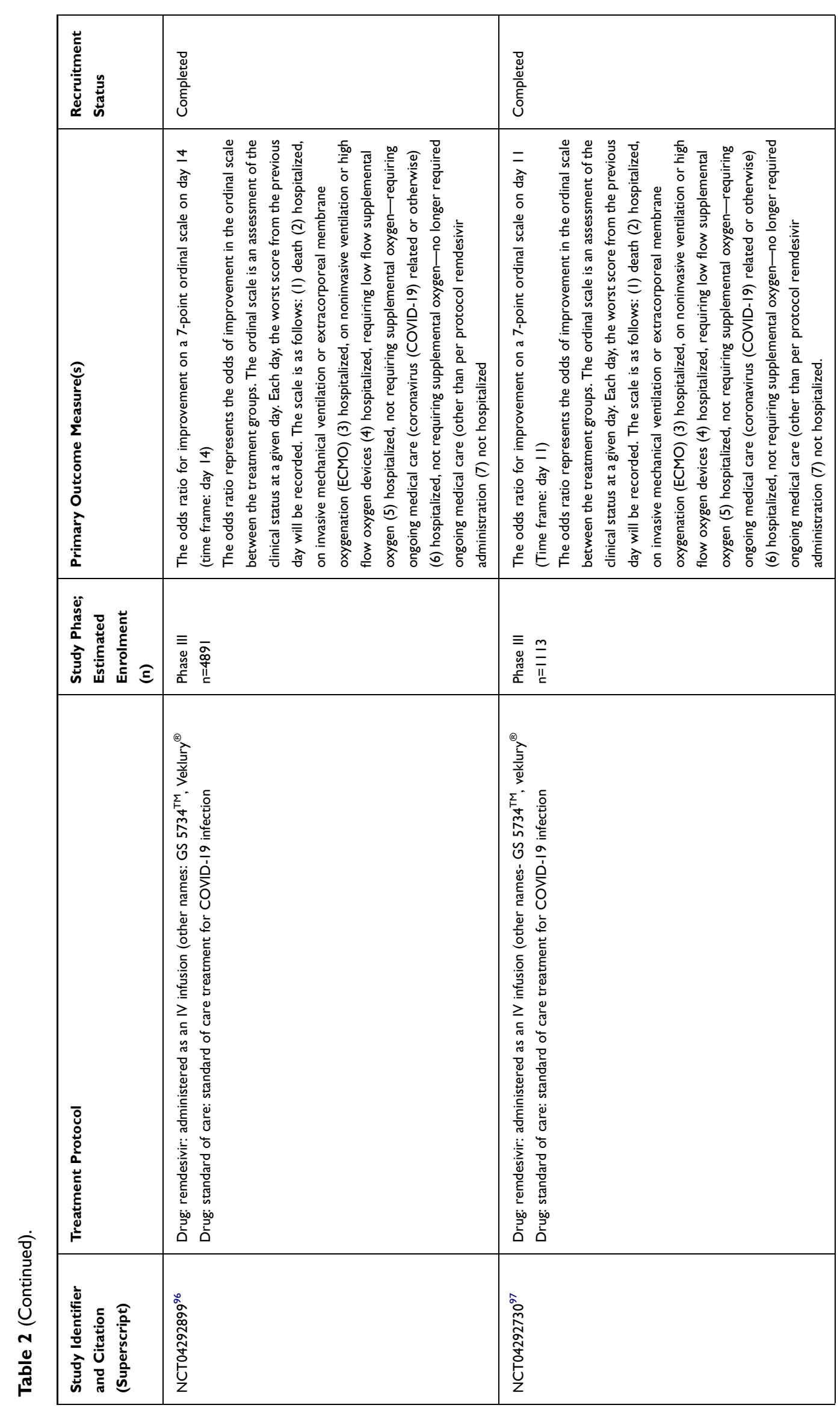




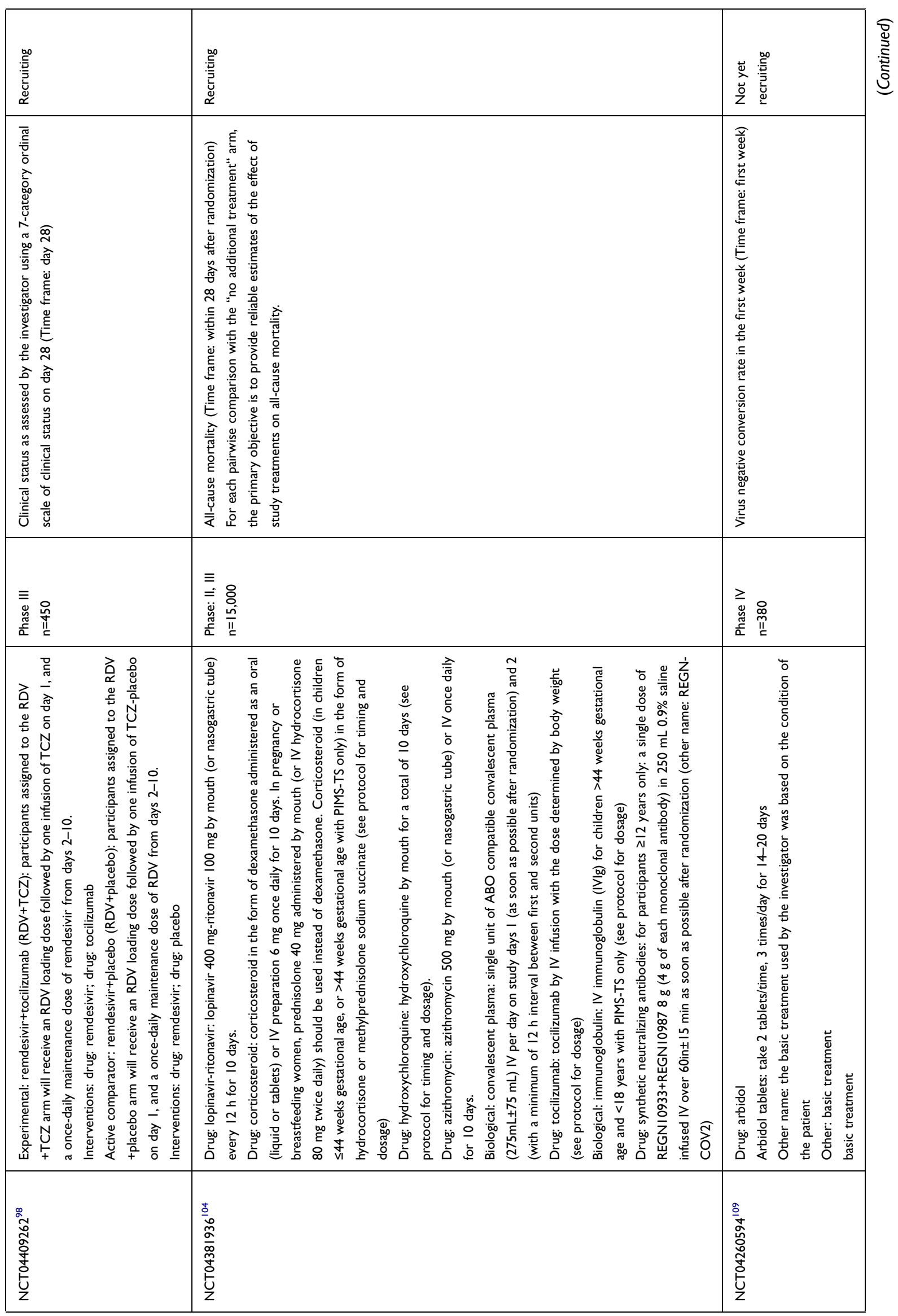




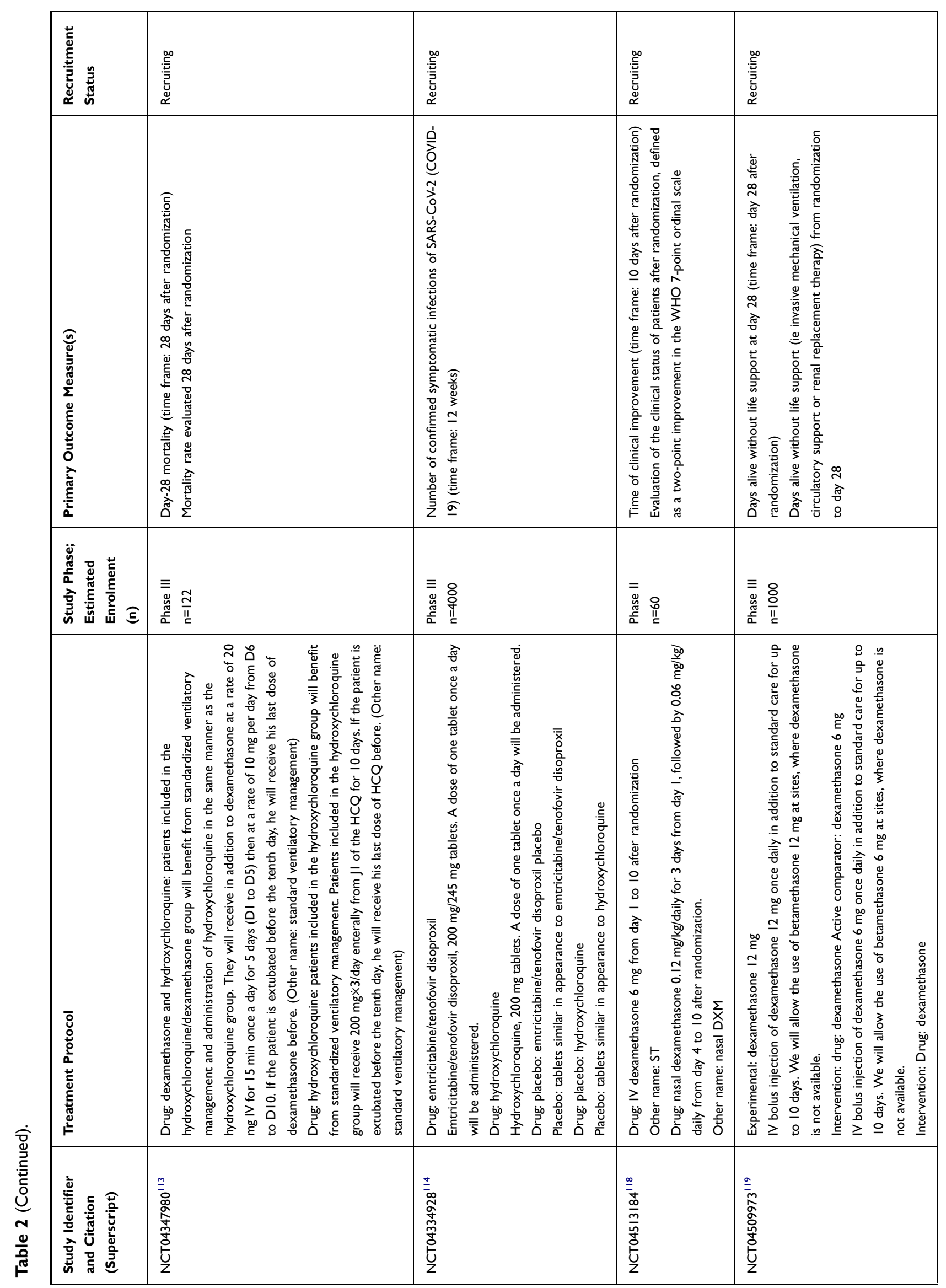




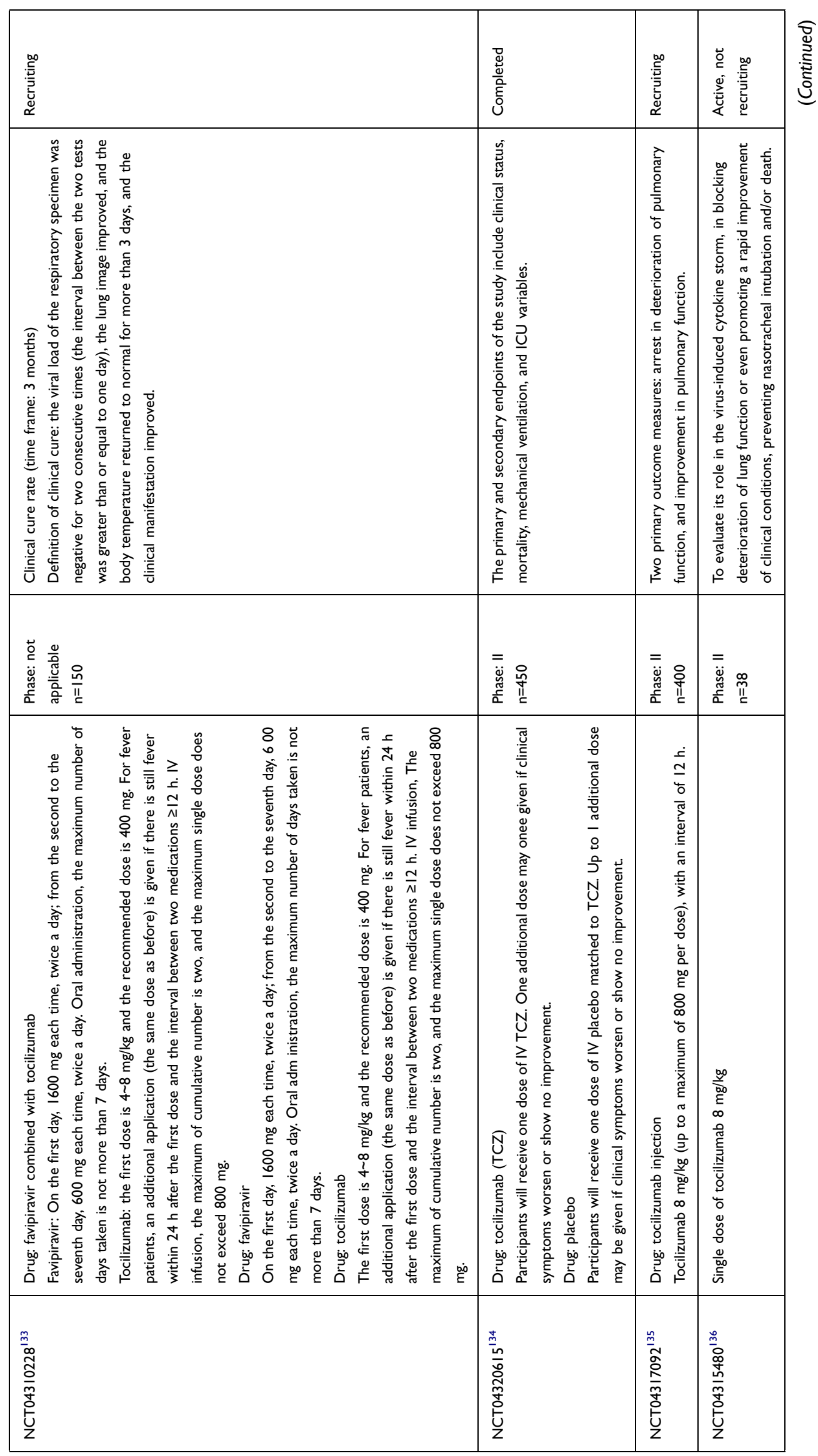




\begin{tabular}{|c|c|c|c|c|c|}
\hline 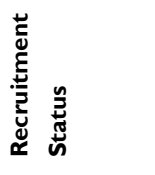 &  & 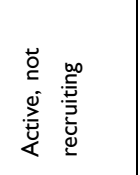 & 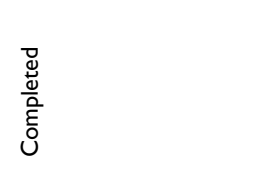 & 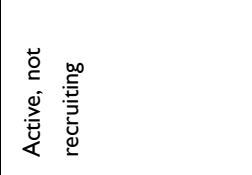 & 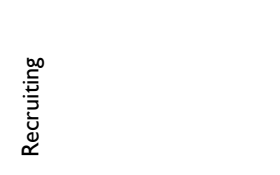 \\
\hline 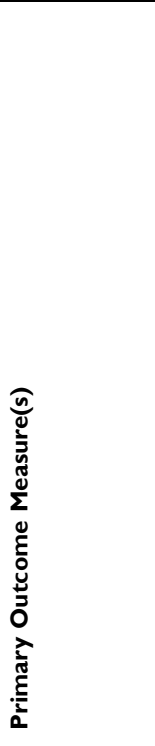 & 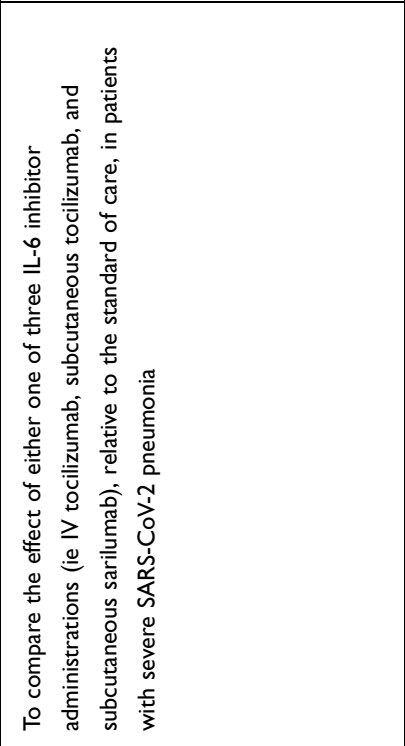 & 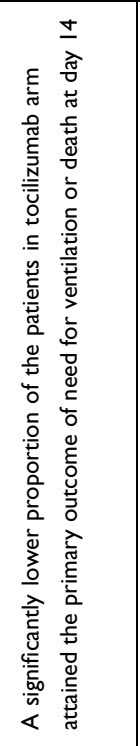 & 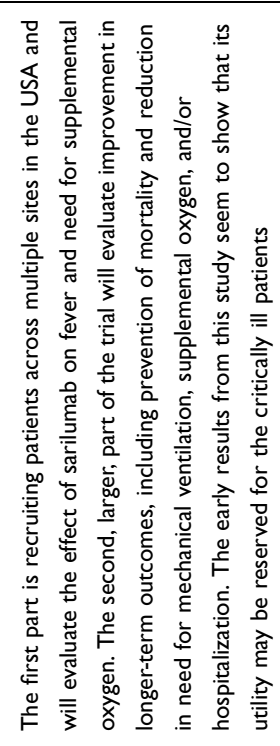 & 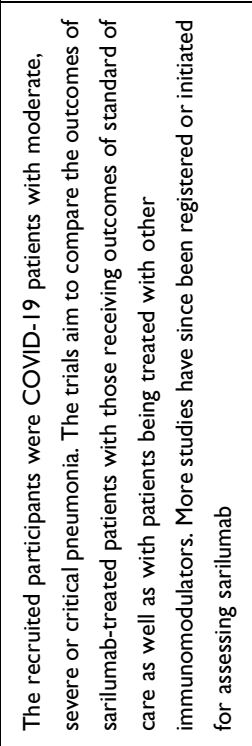 & 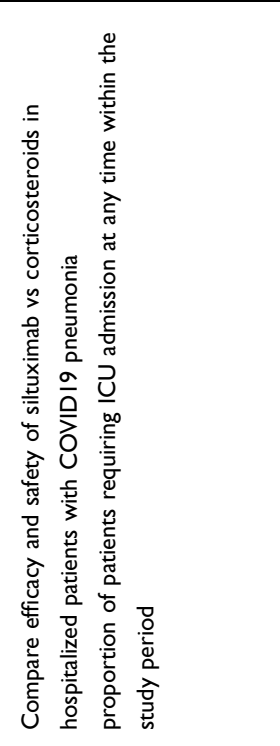 \\
\hline 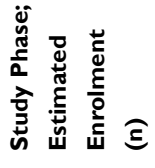 & 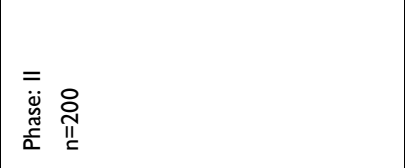 & 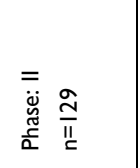 & 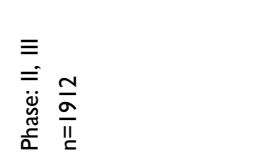 & 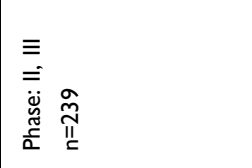 & 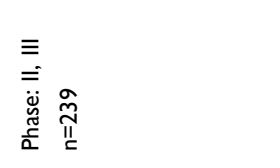 \\
\hline 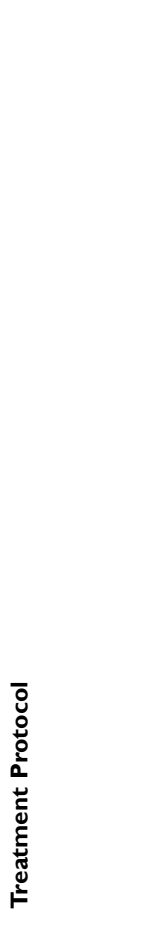 & 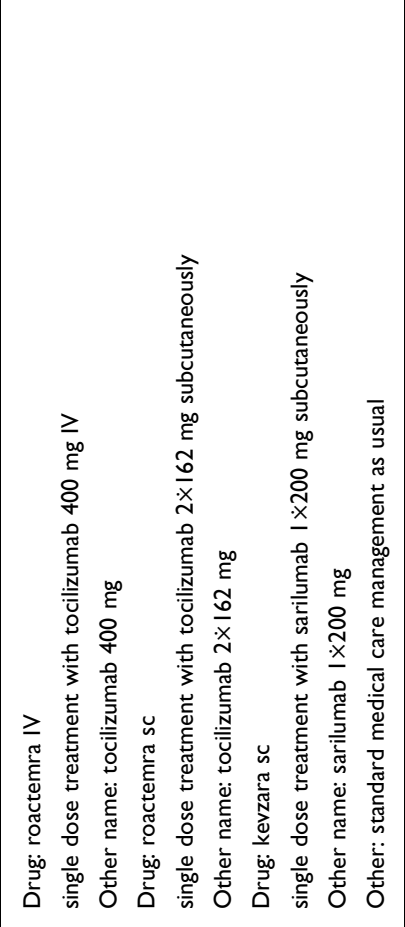 & 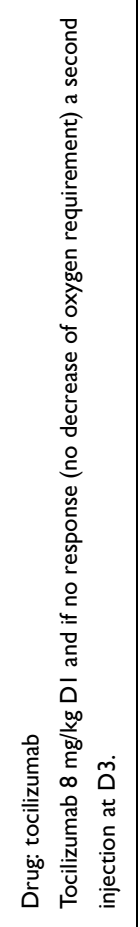 & 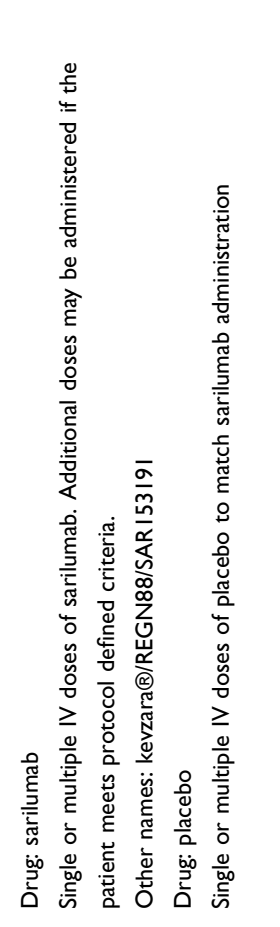 & 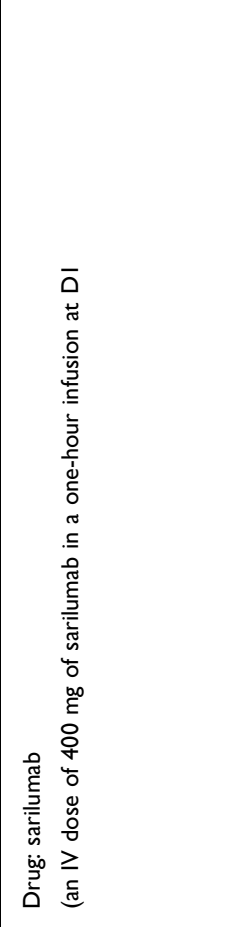 & 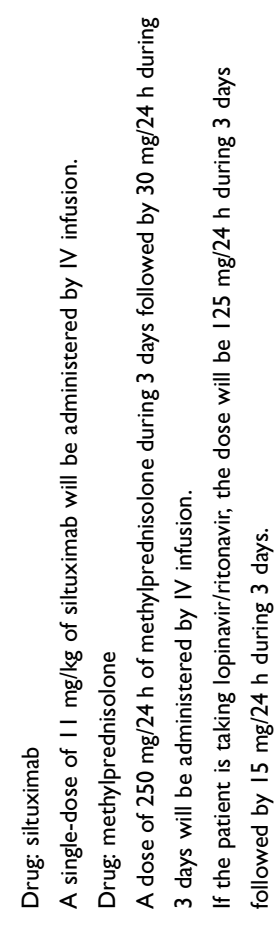 \\
\hline 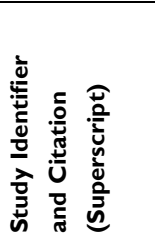 & 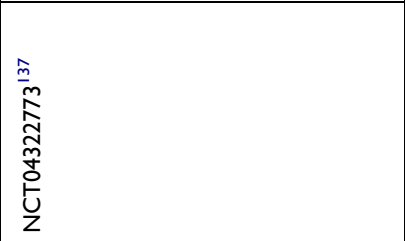 & 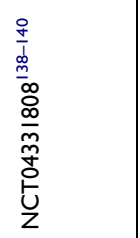 & 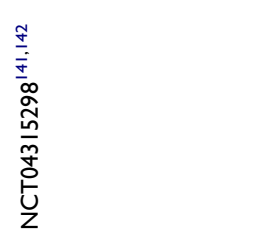 & 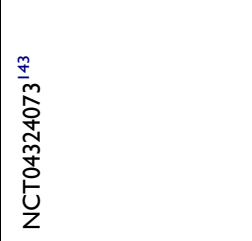 & 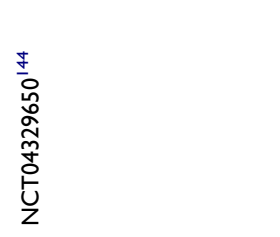 \\
\hline
\end{tabular}




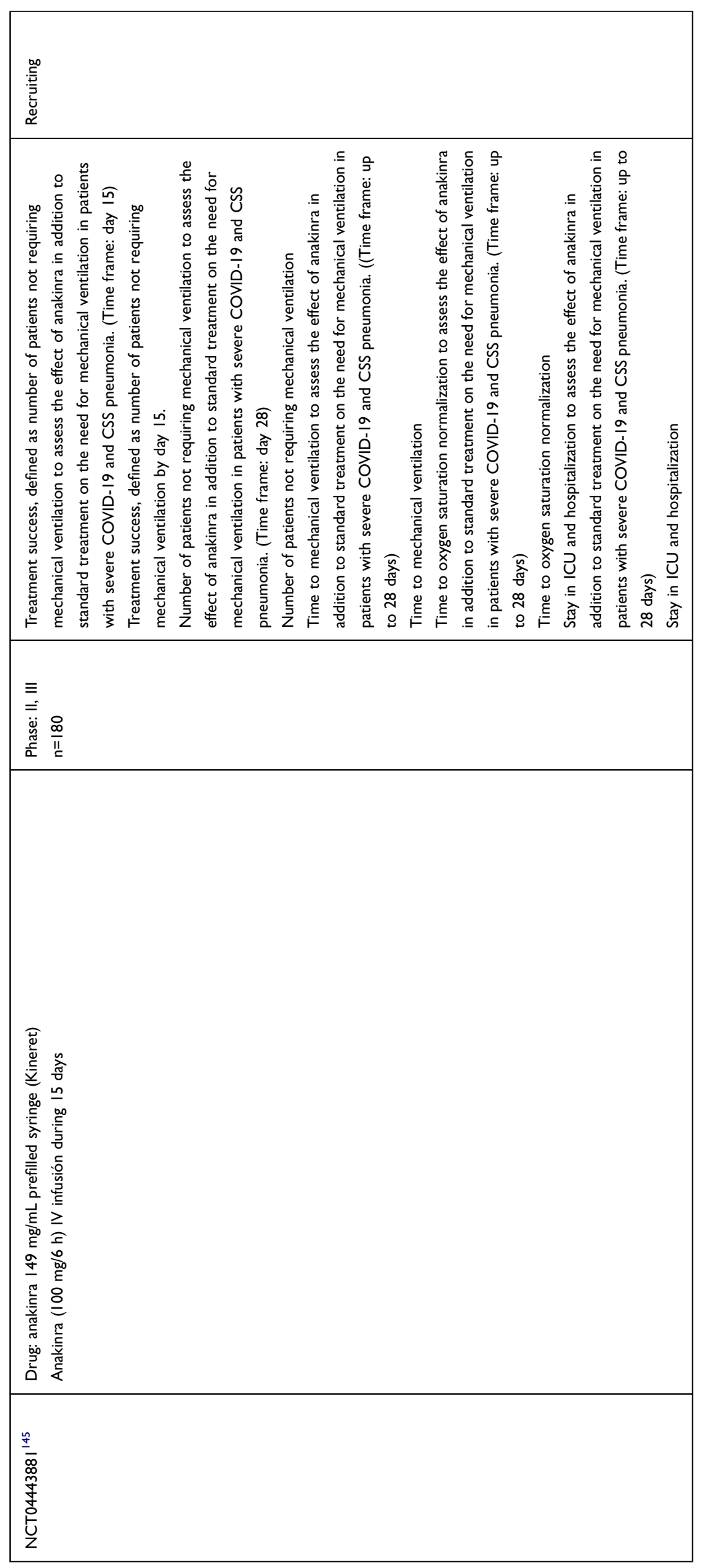


SARS-CoV-2. ${ }^{105}$ It is well-tolerated in children and adults (Table 1). ${ }^{106}$ As a result, it has been one of the recommended antiviral drugs for COVID-19 treatment. ${ }^{107}$ Allergic reactions, nausea, diarrhea, dizziness and elevated serum transaminase are some of the adverse effects associated with this drug (Table 1). ${ }^{106}$ A recent clinical trial was conducted to evaluate the efficacy of using arbidol as a monotherapeutic agent, compared to lopinavir/ritonavir in COVID-19 patients. ${ }^{108}$ The results in this study were in favor of the patients on arbidol monotherapy who recovered in shorter duration period with no apparent side effects, compared to the lopinavir/ritonavir group. ${ }^{108}$ Further use of this drug should be based on safe drug regimens standardized through RCTs, one of which is listed in Table 2. ${ }^{109}$

Chloroquine is a drug extensively used for malarial prophylaxis. ${ }^{110}$ One of its biochemical properties proposes a potent antiviral effect, which has led to its recognition as a broad-spectrum antiviral drug. ${ }^{111}$ In the past, chloroquine has shown beneficial effects in SARS-CoV inhibition by impairing ACE2, ${ }^{102}$ which has also been proven in the in vitro analysis against SARS-CoV-2 ${ }^{111}$ Furthermore, chloroquine is an inexpensive drug and with a good patient safety report and tolerance levels. ${ }^{63}$ Its adverse effects such as, retinal or psychiatric symptoms have been reported only with higher doses. ${ }^{110}$ A systematic review by Cortegiani et al, evaluated the efficacy and safety of chloroquine in COVID-19 treatment. The authors concluded that the drug is effective to reduce SARS-CoV-2 multiplication. Moreover, they suggested the necessity to conduct long-term quality RCTs in the near future to propagate its safe use. ${ }^{112}$ The clinical trials which have evaluated the efficacy chloroquine in COVID-19 patients are highlighted in Table 2. ${ }^{85,104,113,114}$

It can be gathered that all antiviral drugs can play a vital role in the treatment of COVID-19. Nonetheless, to date, there are no approved antiviral drugs for the same. ${ }^{17,74}$ Moreover, the adverse effects of each of them should not be overlooked. Research on the combination of these drugs has shown promising results, and further clinical evaluation of all these drugs is needed through long-term trials. ${ }^{74}$

\section{Corticosteroids}

Corticosteroids have potent anti-inflammatory effects against the COVID-19 cytokine storm (Table 1). ${ }^{115}$ The National Institute of Health and Care Excellence in line with the WHO guidance have confirmed that the dexamethasone (oral or intravenous; IV) and hydrocortisone (IV) play a role in COVID-19 management. ${ }^{116}$ Clinical evidence has shown that glucocorticoids may modulate inflammation-mediated lung injury, which is a key feature of COVID-19 infection, thereby reducing progression to respiratory failure and death. ${ }^{116}$ Dexamethasone $(6 \mathrm{mg}$ once a day; od) can lower the 28-day mortality among those who receive either invasive MV or oxygen alone compared to those receive no respiratory support in the treatment of shock and/or ARDS. ${ }^{116}$ In their a meta-analysis of seven trials, the WHO Rapid Evidence Appraisal for Covid-19 Therapies (REACT) Working Group evaluated corticosteroids (mainly hydrocortisone or dexamethasone) in 1703 critically ill patients in 12 countries and reported that both these drugs reduced the risk of death by about a third, in comparison to the control group. ${ }^{117}$ The RECOVERY (Randomized Evaluation of COVID-19 Therapy) collaborative group have conducted a controlled, open label trial to assess the role of dexamethasone $(6 \mathrm{mg}$ od, oral or IV) for up to 10 days compared to the standard care in 28-day mortality. The primary results of this study demonstrated the beneficial role of dexamethasone in lowering the abovementioned primary outcome compared to the control group (Table 2) ${ }^{104}$ In addition, antishock therapy using small doses of corticosteroids such as hydrocortisone could help in mitigating of CS, which in turn can minimize the occurrence of respiratory distress, septic shock and multi-organ failure, ${ }^{73}$ as well an alternative to dexamethasone in some COVID-19 patients. Some disadvantages include, increased appetite, irritability, difficulty in sleeping (insomnia), swelling in your ankles and feet (fluid retention), heartburn, muscle weakness, impaired wound healing, increased blood sugar levels (Table 1). ${ }^{115,117}$ Other clinical trials based on application of dexamethasone drug therapy in COVID-19 management are listed in Table 2. ${ }^{104,113,118,119}$

\section{Immunotherapy}

Convalescent plasma is plasma rich in antibodies, which is obtained from a patient who has recovered from a virus. Discernibly, the plasma can be taken from patient who has shown a full recovery, and has received ample time to develop a robust antibody response against the virus. The therapy is safe, clinically effective and decreases the mortality rate (Table 1). ${ }^{120,121}$ Convalescent plasma therapy (CPT) has been widely used for diseases like influenza $\mathrm{A},{ }^{122}$ Ebola $^{123}$ and SARS-CoV. ${ }^{124}$ Recently, this form of passive immunization was utilized in COVID-19 management. ${ }^{125}$ The results of this study have shown that CPT was well-tolerated with a significant 
neutralization of the viremia and drastic improvement in the clinical presentation of severely affected COVID-19 patients. ${ }^{125}$ Nevertheless, the benefit of this therapy needs to be established through further large and well-controlled clinical investigations like the one listed in Table 2. ${ }^{104}$ Some drawbacks of this therapy include; transfusion related acute lung injury, transfusion associated circulatory overload and allergic/anaphylactic reactions, transmission of infections, febrile nonhemolytic transfusion reactions, $\mathrm{RBC}$ alloimmunization, and hemolytic transfusion reactions (Table 1). ${ }^{120,121}$

Another immunotherapy is a protective monoclonal antibody $(\mathrm{mAb})$ that targets vulnerable sites of viral surface proteins and deactivates the same, and thus prevents the strain propagation. Recently, researchers isolated the human mAb 47D11, which has demonstrated potential benefits in deactivation of SARS-CoV-2 spike protein. ${ }^{126}$ Although the precise mechanisms of action remain unknown. On this note, the first report on the application of protective $\mathrm{mAB}$ for management of COVID-19 launches a gate for further exploration.

Currently, it is well-known that IL-6 is associated with the inflammation seen in COVID-19 patients and hence IL-6 and IL-6 receptor (IL-6R) inhibition appear to be promising targets to mitigate the adverse effects of this viral infection. Tocilizumab also known as atlizumab, is an anti- interleukin6 receptor humanized $\mathrm{mAb} .{ }^{127}$ It has a potent anti-inflammatory action and is immunosuppressive in nature and has been recommended for the treatment of rheumatoid arthritis and systemic juvenile idiopathic arthritis, a severe form of arthritis in children (Table 1). ${ }^{127-129}$ Lan et al, conducted a systematic review and meta-analysis of seven retrospective studies to assess the efficacy of tocilizumab (IV infusion: 4-8 $\mathrm{mg} / \mathrm{kg}$ over $60 \mathrm{~min}$; if needed repeat at $12 \mathrm{~h}$ max dose $800 \mathrm{mg}$ ), as a potential treatment modality for COVID-19. All-cause mortality of severe COVID-19 patients was lower in the groups receiving tocilizumab, compared to the controls, however, this difference was not statistically significant. Factors such as risk of intensive care unit admission and requirement for mechanical ventilation were similar between the treatment groups. Based on this, the authors concluded that a high quality evidence from future large RCTs data will decide the potential role of this drug in the management of COVID-19. ${ }^{130}$

The use of a novel human anti-interleukin-1 $\beta$ neutralizing $\mathrm{mAb}$ showing in vivo efficacy known as anakinra has surfaced for COVID-19 management in the last few months. Researchers believe that this recombinant IL-1 receptor antagonist can neutralise the hyperinflammatory state, which is considered to be one cause of acute respiratory distress among patients with COVID-19 (Table 1). ${ }^{131}$ A prospective cohort study from Groupe Hospitalier Paris Saint-Joseph (Paris, France) conducted by Huet et al, to assess the off-label use of subcutaneous anakinra (100 mg twice a day for $72 \mathrm{~h}$, then $100 \mathrm{mg}$ daily for seven days) in severely ill COVID-19 patients having symptoms indicative of worsening respiratory function. The group that received anakinra showed 50\% fewer ICU admissions for invasive mechanical ventilation or death, compared to the control group. ${ }^{132}$ Some drawbacks of both these drugs are as follows, headache or dizziness, mouth ulcers, high blood pressure, hypercholesterolemia, allergic reactions, weight gain or swollen ankles, stomach irritation or abdominal pain. The immunosuppressive nature of anakinra may result in an adverse effect on the immune system (neutropenia) and healing, which may lead to reactivation of latent tuberculosis or other atypical or opportunistic infections (Table 1). ${ }^{127-129,131}$ The clinical trials that have been registered to evaluate the efficacy of both these drugs are listed in Table 2. ${ }^{133-145}$

Over the past few months, Regeneron Pharmaceuticals, Inc. have designed an antibody cocktail REGN-COV2, which has been investigated for its potential role in COVID-19 management. Their clinical data have proven that the two antibodies Regeneron selected, REGN10933 and REGN10987, were less likely to generate escape mutants than individual antibodies or other cocktails that have been designed. ${ }^{146}$ The cocktail has demonstrated the following benefits: targeted killing to reduce viral load and the time to alleviate symptoms in nonhospitalized COVID-19 patients, as well in reducing medical visits (Table 1). ${ }^{146-148}$ The University of Oxford in collaboration with Regeneron Pharmaceuticals, Inc. have announced that RECOVERY, which is one of the world's largest RCTs of potential COVID-19 treatments will evaluate Regeneron's investigational antiviral antibody cocktail, REGN-COV2. This will be a phase three open-label trial in hospitalized COVID-19 patients to assess the effects of adding REGN-COV2 to the usual standard-of-care vs standard-of-care on its own (Table 2). ${ }^{104}$ This antibody cocktail has been chosen by the university owing to its emerging safety profile in humans, preclinical data showing it could protect against viral escape mutations and reduce the amount of virus and associated damage in the lungs as demonstrated successfully in in vitro and in vivo nonhuman primate treatment studies. ${ }^{104,146}$ Other antibody cocktail therapies that have been announced 
are by Eli Lilly with AbCellera-partnered LY-CoV555, AstraZeneca with researchers from Vanderbilt University and a collaboration in between GlaxoSmithKline and Vir Biotechnology and all trials of the above are in difference phases at present. ${ }^{147}$ Use of systemic anti-inflammatory drugs such as baricitinib to reduce the adverse effects of the cytokine storm has also been assessed, due to its anti-inflammatory and antiviral effects. ${ }^{148}$

\section{Prospective Therapies for COVID- 19 Management}

Apart from the various treatment options mentioned above, there are some upcoming modalities that have emerged, as prospective alternatives and the corresponding registered clinical trials are listed in Table $3 .{ }^{149-168}$ These include:

1. Gheblawi et al, in their review have highlighted the role of ACE2 as the novel SARS-CoV-2 receptor and have provided a critical link between immunity, inflammation, ACE2, and cardiovascular disease. ${ }^{169}$ The authors have stated that the action of ACE2 is enhanced by recombinant ACE2, gene-delivery of Ace2, Ang 1-7 analogs, and Mas receptor agonists. ${ }^{169}$ The latter serves as potential therapies for disease conditions associated with an activated RAS (Table 3). ${ }^{149,150}$

2. Molecular mechanism of exosome-based (30-120 nm extracellular vesicles) strategies are being tested to fight the COVID-19 infection (Table 3). ${ }^{151,152}$ These vesicles help in intercellular communication via delivering biomolecules like nucleic acids, proteins, and lipids to recipient cells (Table 1). ${ }^{170,171}$ They contain receptors for viruses (CD9 and ACE2) that make recipient cells susceptible to virus entry. They are released by virus-infected cells and are associated with the transfer of viral components. ${ }^{170}$ Researchers believe that exosomes have a role in the spread of the SARS$\mathrm{CoV}-2$ virus and a thorough understanding of their relation to the COVID-19 infection could aid in identifying vital information regarding the entry, replication and spread of the virus in order to combat its adverse effects (Table 1). ${ }^{170,171}$ However, some drawbacks that have been noted with regards to this therapy are as follows: inefficient separation methods, difficulties in characterization and lack of specific biomarkers. At present, the strategies that are being tested include; inhibition of exosome biogenesis and uptake, exosome-therapy, exosome-based drug delivery system, and exosomebased vaccine (Table 3). ${ }^{151,152}$
3. Cellular therapy: an intravenous transplantation of ACE2-mesenchymal stem cells (MSCs) or natural killer cells to mediated-antiviral therapy. ${ }^{21,172}$ MSCs are able to suppress the activities of viruses via Chafla-mediated and Sumo2-mediated epigenetic regulation (termed proviral silencing) and have demonstrated beneficial effects in ARDS. However, there is a lack of clarity with regards to optimal dose and route of MSC delivery, difficulties in large-scale production and cryopreservation, and the potential for substantial variability (Table 1). ${ }^{172,173}$ Table 3 provides data on three clinical trials which have been registered for assessed MSCs in COVID-19 management. ${ }^{153-155}$

4. As the ACE2 is a vital component of renin-angiotensin system (RAS), it can be a possible treatment by utilizing the RAS inhibitors such as; ACE-inhibitors (ACEI) and angiotensin II receptor type 1 (AT1R). ${ }^{174}$ These drugs are not associated with an increased risk of death, admission to intensive care unit (ICU), mechanical ventilation requirement or progression to severe or critical pneumonia in COVID-19-infected hypertensive patients and they can be used safely in children and adults. There is insufficient evidence of their potential harm and only overwhelming evidence shows their benefits, thus favoring their utilization in COVID-19 management in the clinical trials listed in Table $3 .^{156-158}$

5. Traditional Chinese medicines (TCMs) such as; qingfei paidu decoction (QPD), gancaoganjiang decoction, sheganmahuang decoction, qingfei touxie fuzheng recipe, glycyrrhizin, hesperetin, baicalin and quercetin, are rich in naturally occurring flavonoids. ${ }^{175,176}$ The advantages of TCMs include, remarkable symptomatic relief, antipyretic, faster recovery, reduced hospital stay results in their utilization in previous epidemics. However, some commonly associated drawbacks include, hypersensitivity pneumonitis, lung injury, fatigue with gastrointestinal discomfort (Table 1). ${ }^{175-178}$ Table 3 lists two important clinical trials that have been registered to assess TCMs, as a potential treatment strategy in COVID-19 patients. ${ }^{159,160}$

6. Consumption of food products rich in vitamins A, C, $\mathrm{D}$, and $\mathrm{E}$, which are naturally occurring antioxidants. ${ }^{73}$ They can prevent or reduce the damage caused by oxidation as well as reduce the risk of cardiovascular disease. Some drawbacks include; bruising under the skin, diarrhea, dizziness, joint pain (Table 1). ${ }^{179,180}$ Clinical trials which have been registered to evaluate antioxidant therapy in COVID-19 are listed in Table 3. ${ }^{161-164}$ 


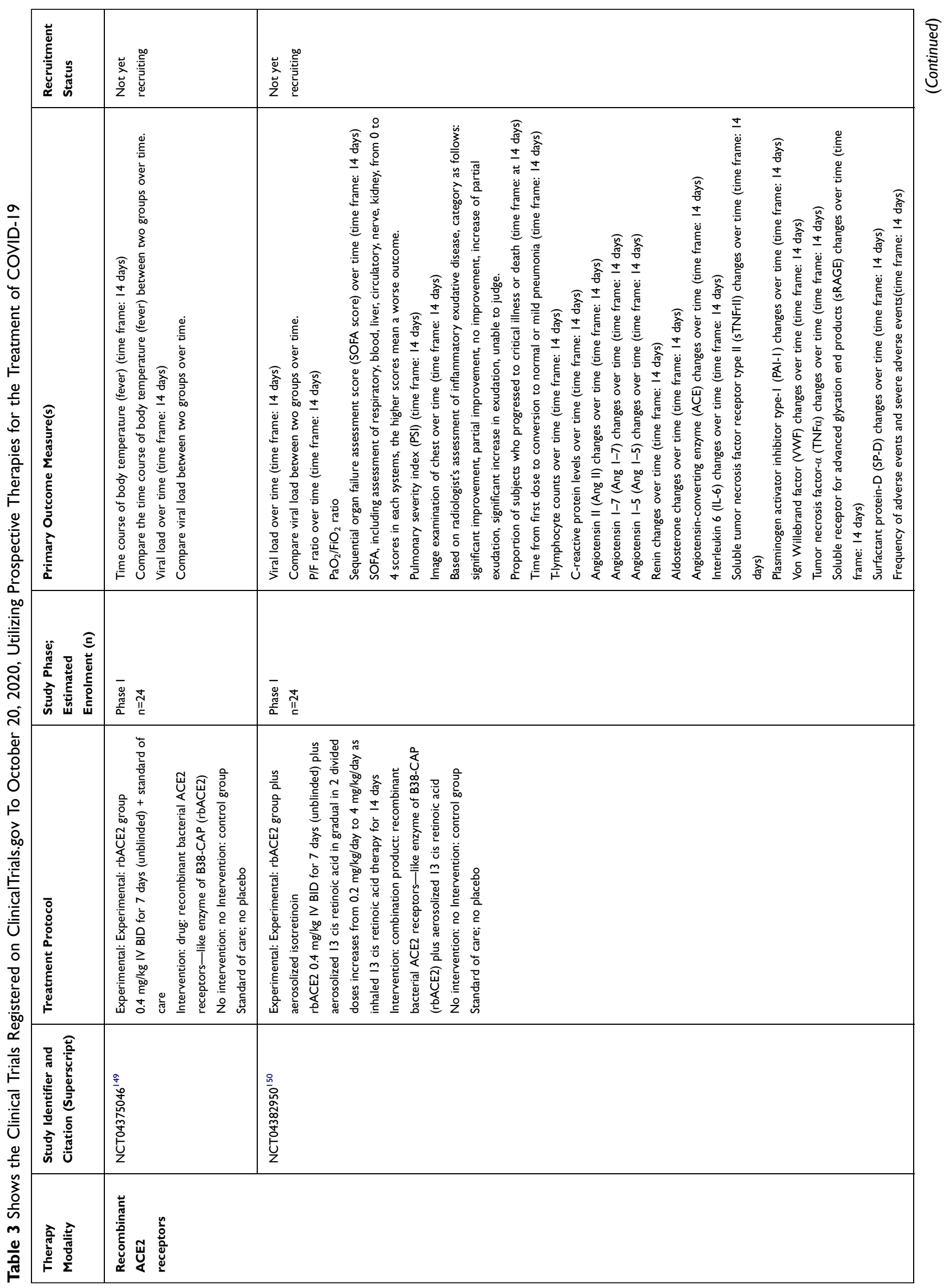




\begin{tabular}{|c|c|c|c|}
\hline 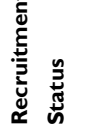 & 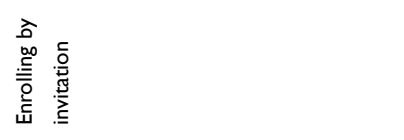 & 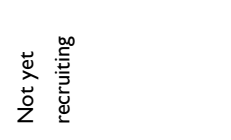 & 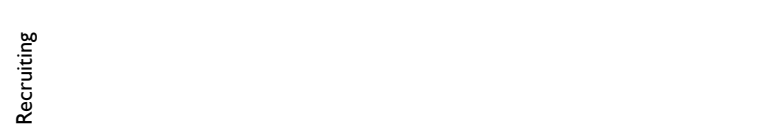 \\
\hline & 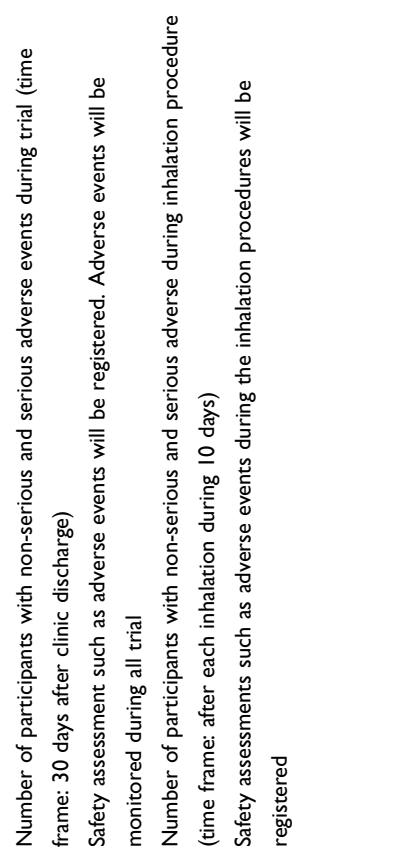 & 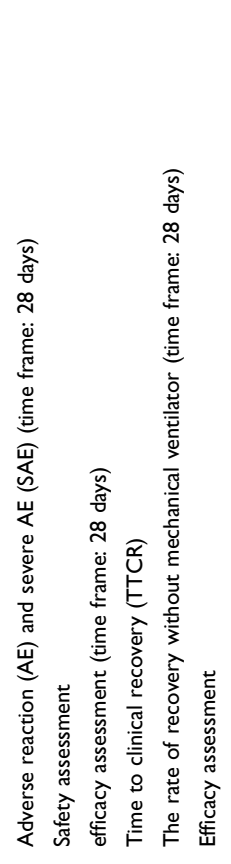 & 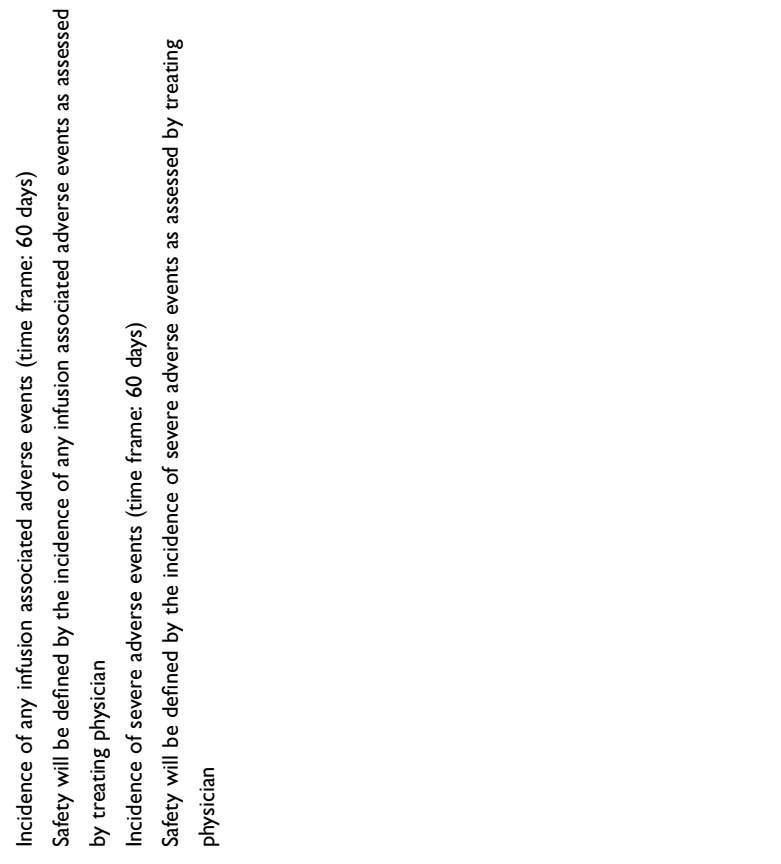 \\
\hline 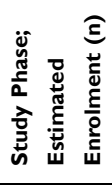 & 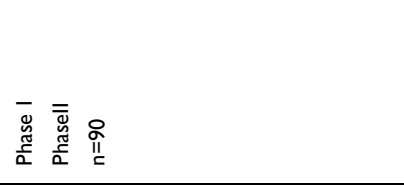 &  & 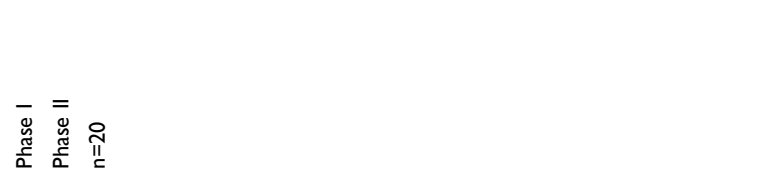 \\
\hline & 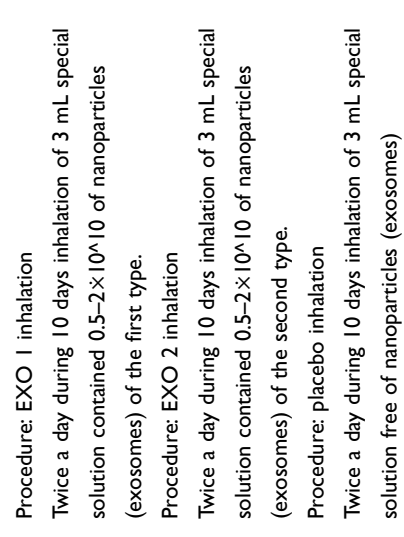 & 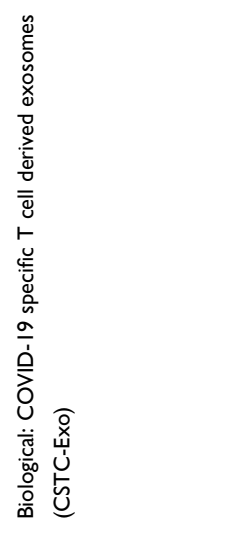 & 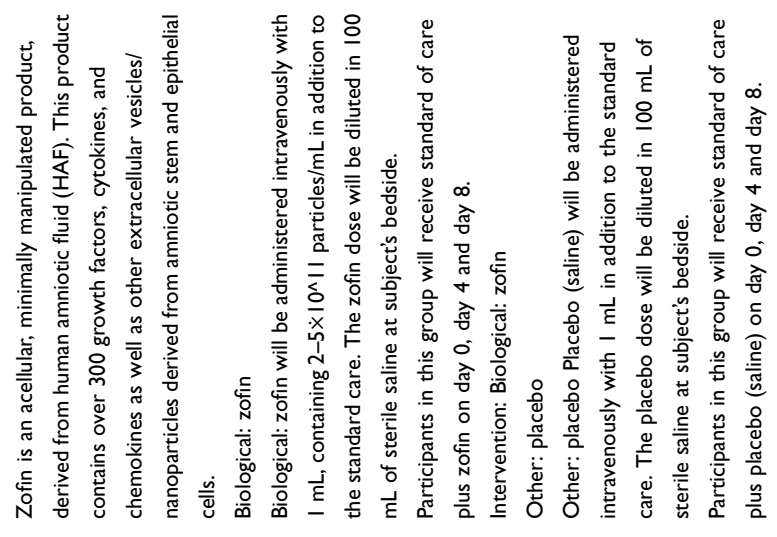 \\
\hline 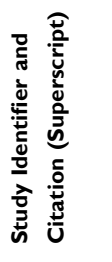 & 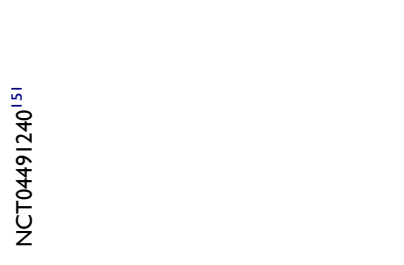 & 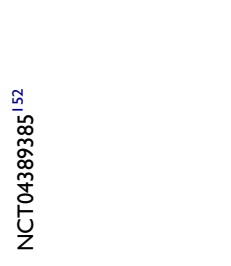 & 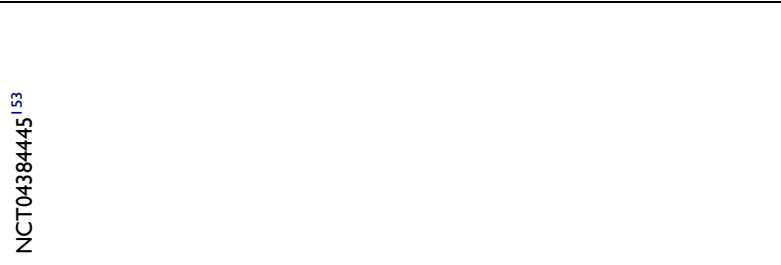 \\
\hline 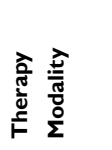 & 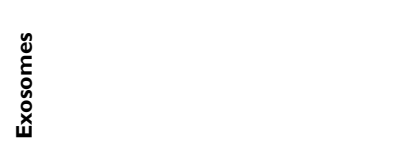 & & $\begin{array}{l}\dot{u} \\
\dot{m}\end{array}$ \\
\hline
\end{tabular}




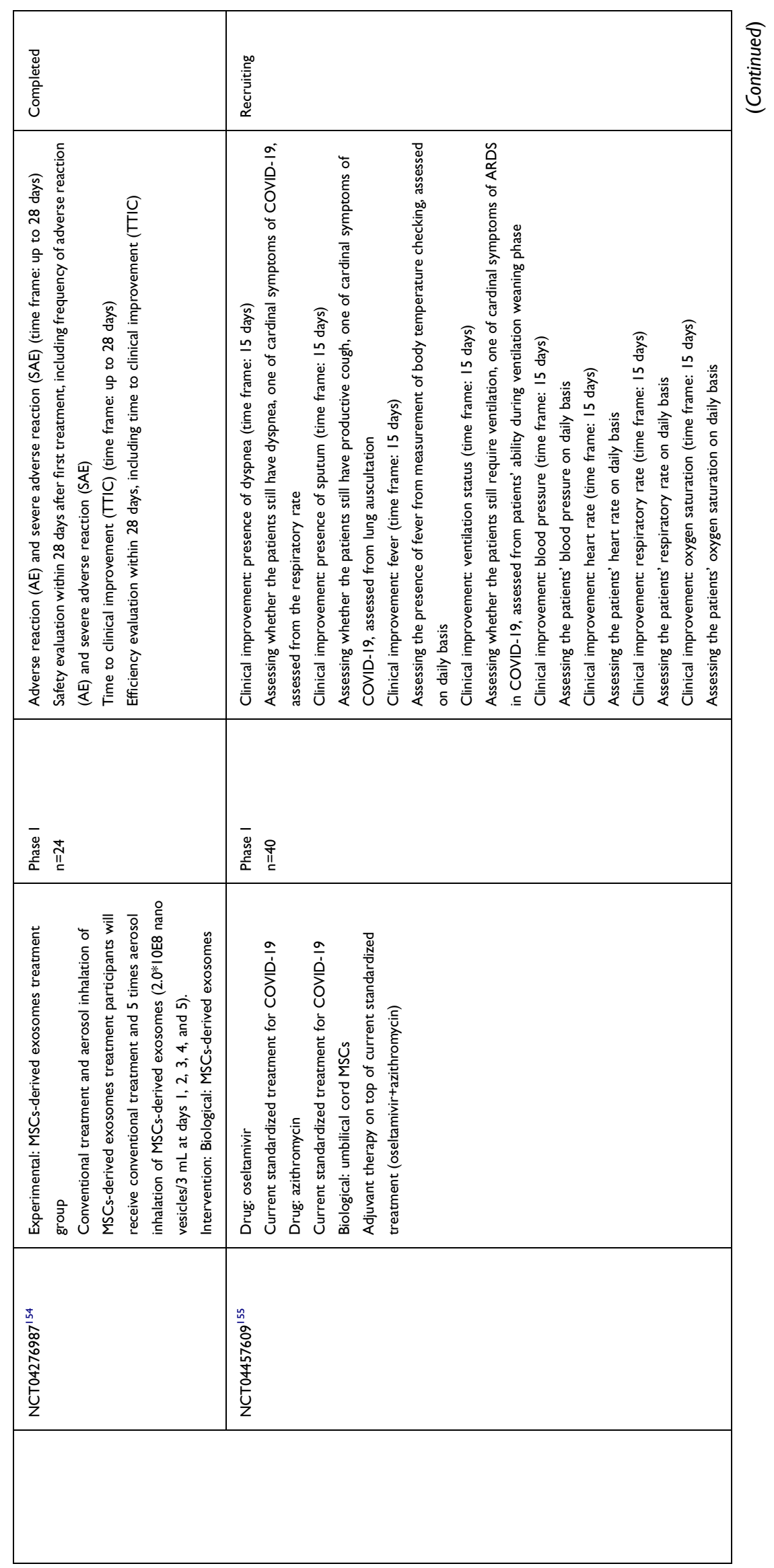




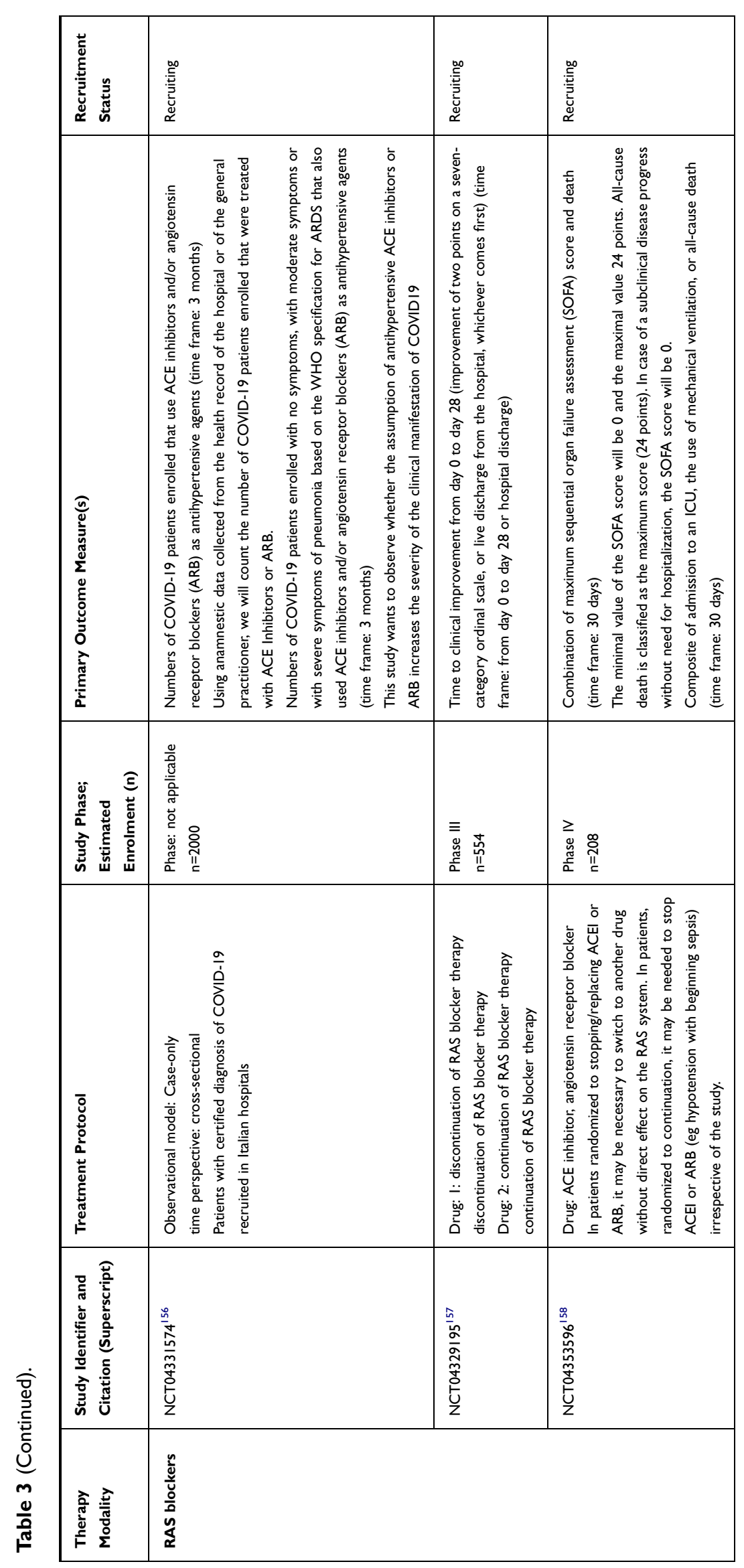




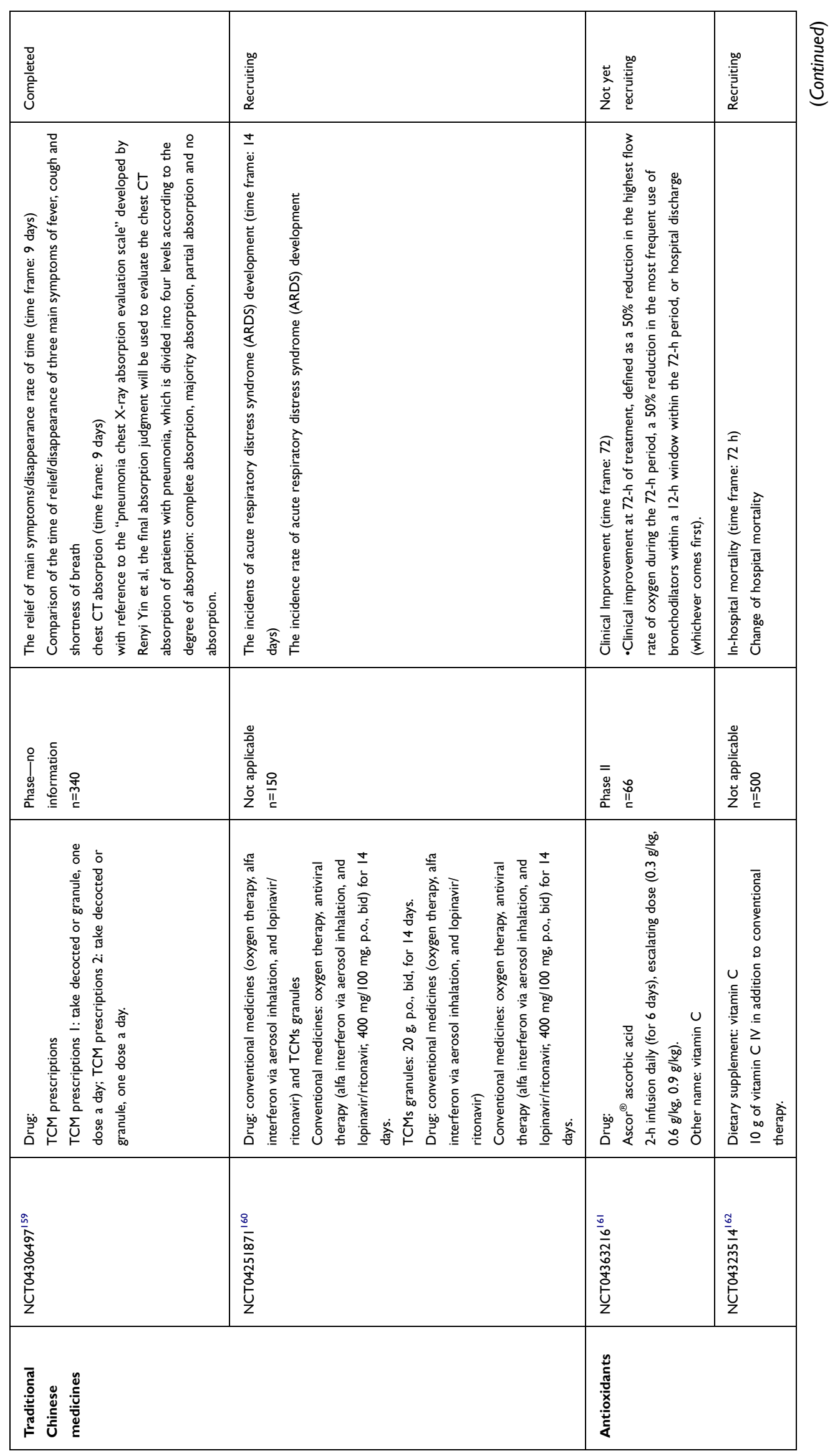




\begin{tabular}{|c|c|c|}
\hline 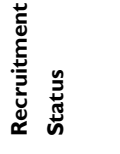 & 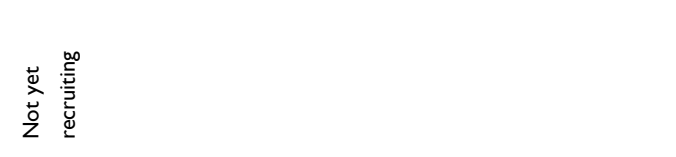 & 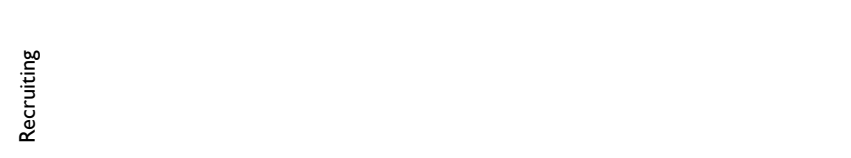 \\
\hline & 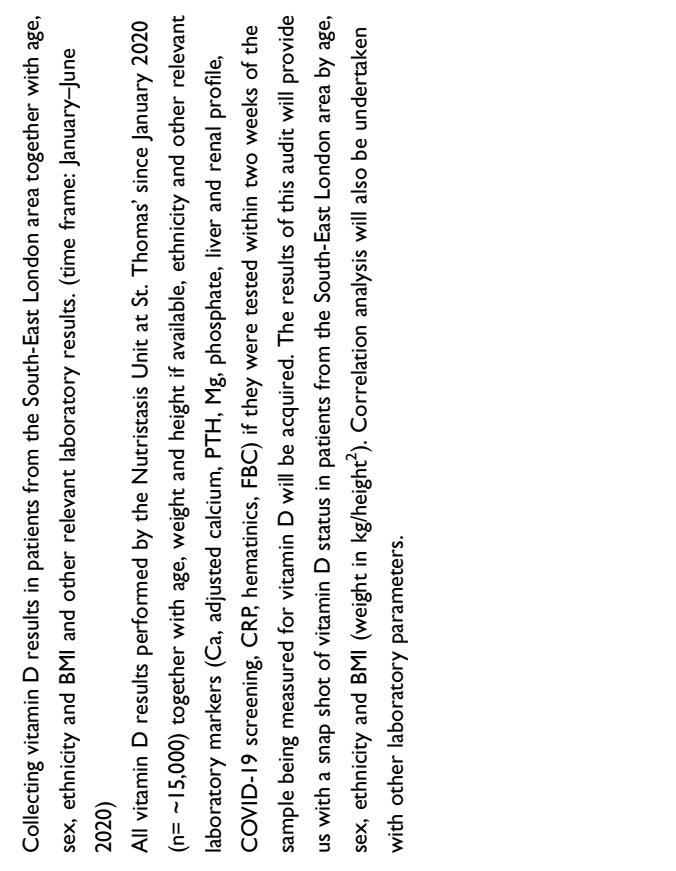 & 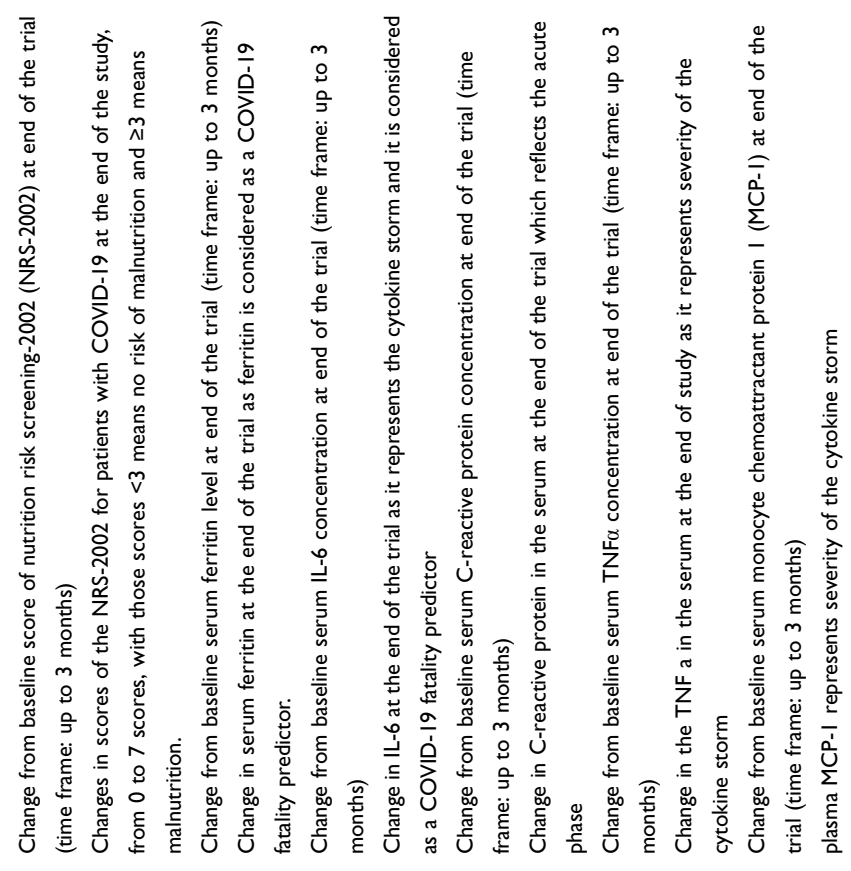 \\
\hline 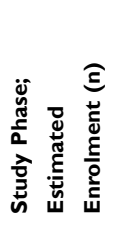 & 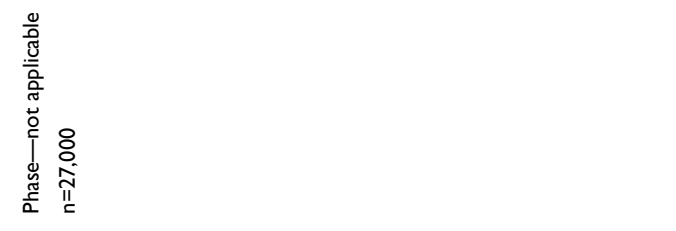 & 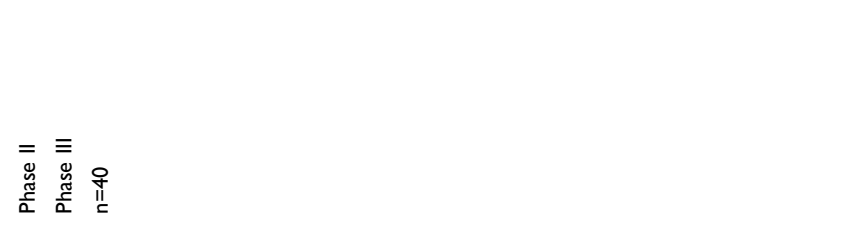 \\
\hline & 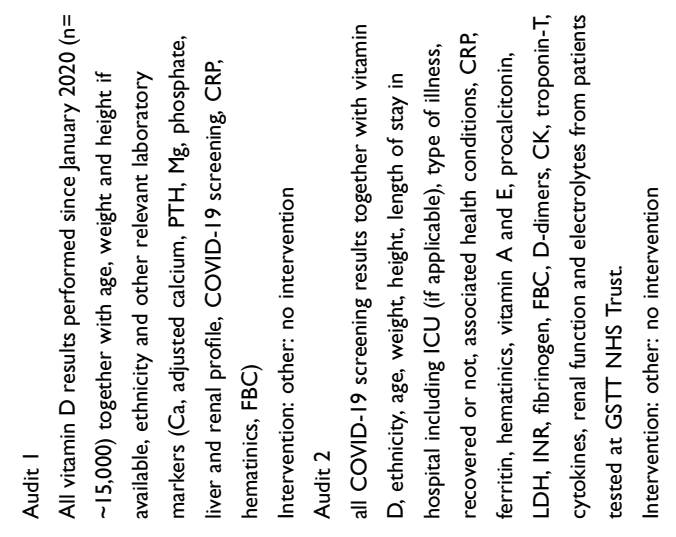 & 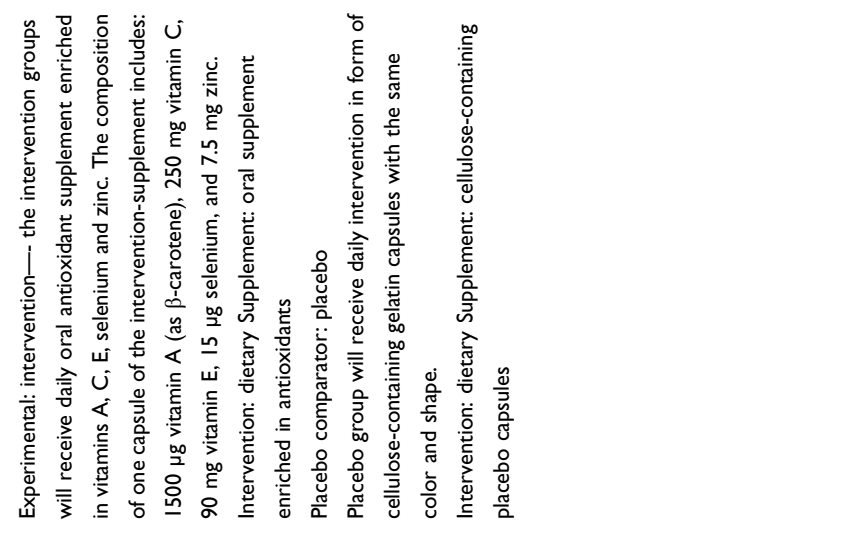 \\
\hline 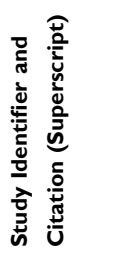 & 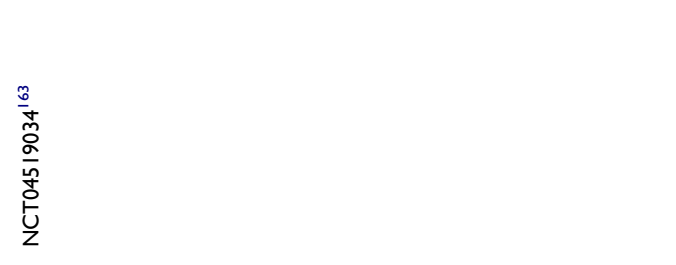 & 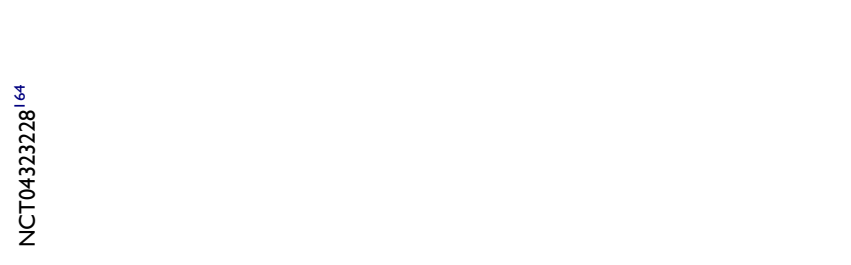 \\
\hline 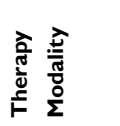 & & \\
\hline
\end{tabular}




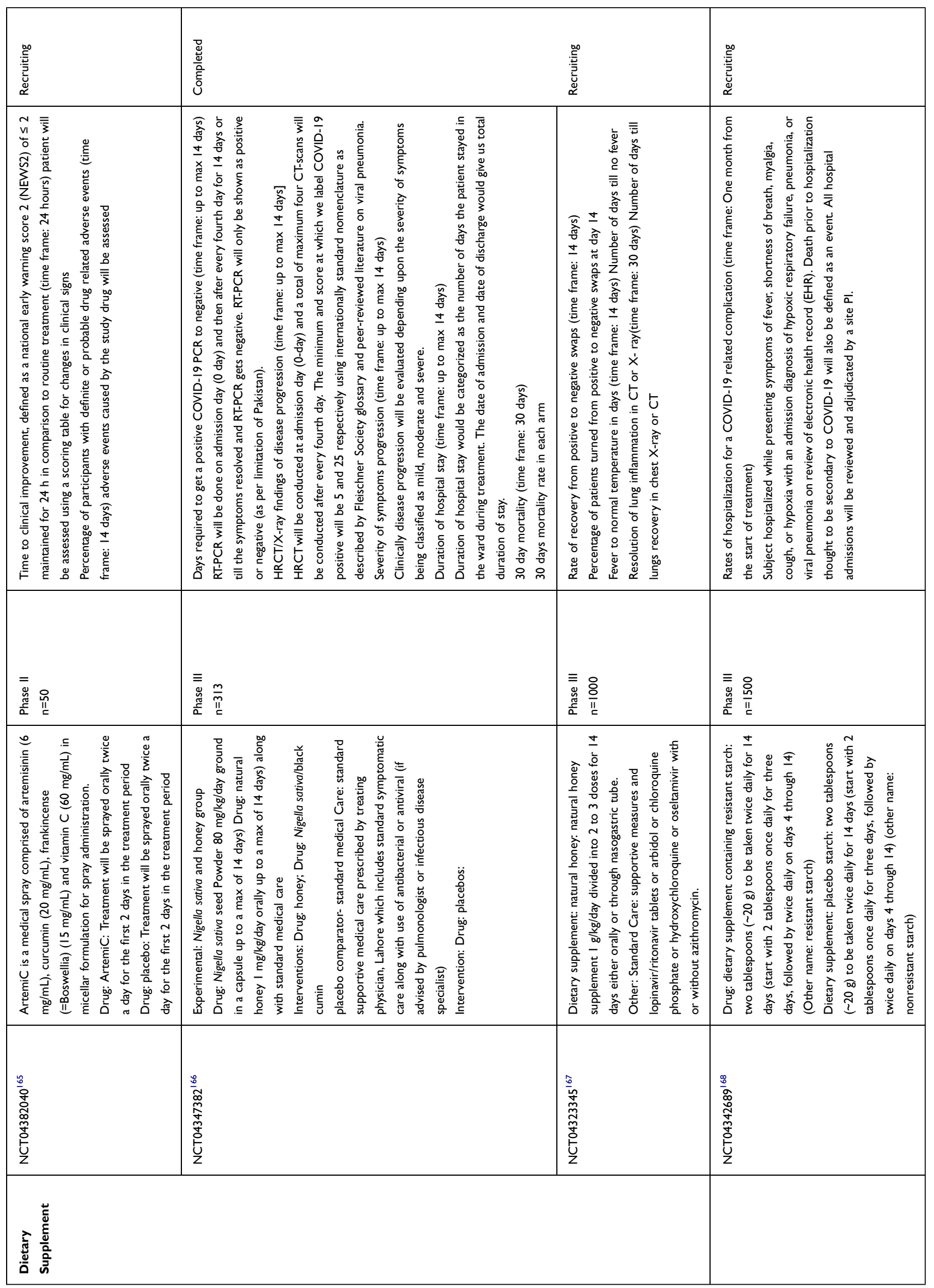


7. Curcumin may have a potential role against COVID19 , owing to its scientifically proven benefits such as its ability to modulate various molecular targets that contribute to the attachment and internalization of SARS-CoV-2 in various human organs, cellular signaling pathways and RNA replication, as well its role in suppression of pulmonary edema and fibrosis-associated pathways (Table 1). ${ }^{181}$ It has also demonstrated strong inhibitory effects on NF- $\mathrm{kB}$ and several pro-inflammatory cytokines for which it can be used, as an adjunct in reversing the fatal CS that occurs in severely-ill COVID-19 patients. ${ }^{181}$ Owing to no known drawbacks of curcumin it is being assessed in a clinical trial (Table 3). ${ }^{165}$

8. The role of omega-3 fatty acids, honey and its various components as well as potato starch have been highlighted as novel natural therapies to fight against the symptoms of COVID-19 infection. ${ }^{182}$ These therapies are known to have a potent antibacterial and anti-inflammatory effects (Table 1). ${ }^{183-185}$ Methylglyoxal (MGO) is a component of manuka honey which can inhibit enveloped virus growth and inhibit SARS-CoV-2 replication. ${ }^{184,186} \mathrm{In}$ addition, the higher the concentration of MGO, the stronger the antibiotic effect. There are no known drawbacks of honey and starch, however, the omega 3 fatty acids namely, eicosapentaenoic acid (EPA) and docosahexaenoic acid (DHA) can make cell membranes more susceptible to nonenzymatic oxidation mediated by ROS, leading to the formation of potentially toxic oxidation products and increasing the oxidative stress (Table 1). ${ }^{183-185}$ Researchers believe that these natural products can help boost the host immune system, improve comorbid conditions and antiviral activities in COVID-19 patients and hence some clinical trials have been registered to assesses their outcomes (Table 3) ${ }^{16-168}$

\section{Vaccine}

Presently, the entire world has joined hands and engaged all the available resources and manpower to develop a vaccine against COVID-19. It is believed that vaccination could be acknowledged as the epitome of all existing remedies assigned for controlling the COVID-19 outbreak. Quite a large number of vaccines are undergoing trials across different parts of the world to fight against the present pandemic situation. ${ }^{73,74}$ Epitopes, mRNA and $\mathrm{S}$ protein-receptor binding domain structure-based vaccines are the most coming vaccine proposals, which have been initiated. ${ }^{75}$ However, scientists have estimated that the process is time consuming and consisting of sequential phase-wise trials with quite a few safety evaluations. Notably, this entire process will take almost 12-18 months in achieving optimal results, as well as mass manufacture. ${ }^{76}$ Moreover, after the vaccine is available to the general population, human clinical trials would be required to prove the efficacy and safety of the same. The efforts of the entire scientific fraternity in the fight against this brutal fatality have just started materializing. Newer epidemiological facts, properties of the virus, immune responses against the virus and challenges in vaccine production are surfacing each day. ${ }^{50}$ Evidently, the efforts invested into research have to be amplified with an urgent application in order to combat this disease. Perhaps this is how research will evolve and the vaccine will be developed.

\section{Future Noninvasive Therapy}

COVID-19 remains a serious concern, until now, as there are a lack of effective antiviral drugs and the ongoing studies are yet to produce a safe and effective vaccine. Hence, phototherapy could be considered to treat COVID-19, as a therapeutic or preventive treatment modality. The term phototherapy encompasses photobiomodulation therapy (PBMT), including lasers light and laser-emitted diodes (LEDs) and photodynamic therapy (PDT). ${ }^{187,188}$ It is welldocumented in the scientific literature that phototherapy presents a promising noninvasive treatment modality in tissue healing and regeneration, as well in pain management. ${ }^{189-194}$

\section{The Rationale for Use of PBMT (Laser and LEDs) in COVID-19}

The role of PBMT in modulating the molecular and cellular activities plays a significant part in achieving the optimal therapeutic outcomes. ${ }^{190,195}$ Experimental studies utilizing murine models of acute airway and lung inflammation have shown that PBMT reduces pulmonary microvascular leakage, IL-1 $\beta$, IL-6, and intracellular ROS. On this note, PBMT as a single or adjunct treatment modality can modulate the cytokine storm and ARDS via its antiinflammatory action ${ }^{196-199}$ and this has demonstrated the potential effect of PBMT in reducing the need for ventilators in patients with COVID-19. ${ }^{199,200}$

Several in vivo animal studies have utilized various wavelengths such as; $\lambda 650 \mathrm{~nm}, \lambda 660 \pm 20 \mathrm{~nm}^{201}$ and $\lambda$ $780 \mathrm{~nm}$ lasers for PBM irradiation in which positive results reported as follows: downregulation of inflammatory mediators, reduction in the activation of neutrophils influx, improvement in the endothelial damage, reduction in TNF $\alpha$, and IL-1 $\beta$ in the lung, as well lung edema. ${ }^{201-203}$ 
On this note, ARDS symptoms, as a result of SARS-CoV2 infection can be improved with PBMT. ${ }^{204}$ Additionally, in vivo experimental animal model studies of respiratory disease suggested that PBMT reduces inflammation and promotes lung healing. ${ }^{199,205,206}$ These in vivo animal studies have shown PBMT effectiveness in minimize the length of time needed on a ventilator, enhance the healing process, and shorten recovery time. As a result, this could significantly reduce severely overwhelmed health-care systems. Hence, there is an urgency of proposing this therapy for COVID-19 management. ${ }^{207}$ This statement is supported by the evidence-based clinical results, which showed a significant improvement in patients with chronic obstructive pulmonary syndrome (COPD) and asthma following PBMT. ${ }^{208,209}$

Figure 3 illustrates the mechanism in which PBM can reverse the cellular and molecular activities of the SARSCoV-2 virus. ${ }^{200,203,206}$ On this note, the first severe case of COVID-19 pneumonia treated with PBMT, as an adjunct to standard conventional protocol was recently treated by Sigman et al, which aimed to reduce inflammation and promote lung healing. The presentation of this case is related to a 57-year-old African American man with severe COVID-19 who, once-daily for four consecutive days, received PBMT sessions by a laser scanner with pulsed $\lambda$ $808 \mathrm{~nm}$ (peak power of $3 \mathrm{~W}$, frequency $1500 \mathrm{~Hz}, 330$ microseconds, $50 \%$ duty cycle) and super-pulsed $\lambda 905$ $\mathrm{nm}$ (peak power $75 \mathrm{~W} \times 3$, frequency $1500 \mathrm{~Hz}, 10$ nanoseconds) for $28 \mathrm{~min}$ (14 mins for each lung). ${ }^{210}$ The fluence was $7.2 \mathrm{~J} / \mathrm{cm}^{2}$ and the total energy $3600 \mathrm{~J}$. The laser device utilized scanner, which positioned $20 \mathrm{~cm}$ above the target tissue and scanned $250 \mathrm{~cm}^{2}$ over each lung. The patient was evaluated before and after treatment via radiological assessment of lung edema (RALE) by CXR, pulmonary severity indices, blood tests, oxygen requirements and patient questionnaires. The results have shown that oxygen saturation $\left(\mathrm{SpO}_{2}\right)$ has increased from $93-94 \%$ to 97-100\%, whilst the oxygen requirement has decreased from $2-4 \mathrm{~L} / \mathrm{min}$ to $1 \mathrm{~L} / \mathrm{min}$. The RALE score improved from eight to five. Furthermore, the pneumonia severity index (PSI) improved from Class V (142) to Class II (67). Furthermore, both pulmonary indices (Brescia-COVID and SMART-COP) were decreased from four to zero. The CRP normalized from 15.1 to 1.23 . The patient has
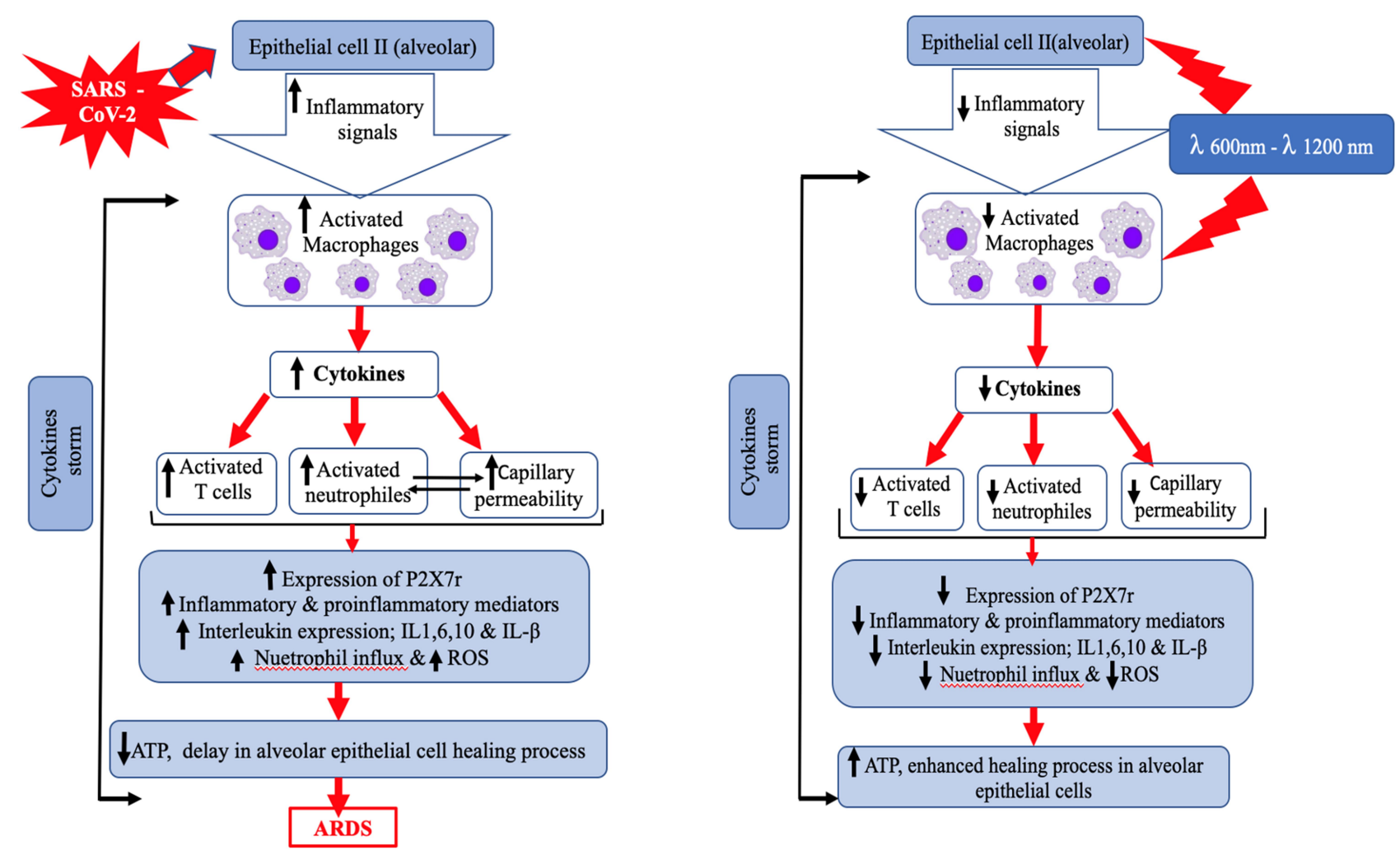

Figure 3 Modified schematic diagram illustrates the cellular and molecular cascade of PBMT that reserve SARS-CoV-2 cellular and molecular activities to prevent ARDS. Notes: Adapted with permission from Mokmeli S, Vetrici M. Low level laser therapy as a modality to attenuate cytokine storm at multiple levels, enhance recovery, and reduce the use of ventilators in COVID-19. Can J Respir Ther. 2020;56:1-7. ${ }^{200}$ 


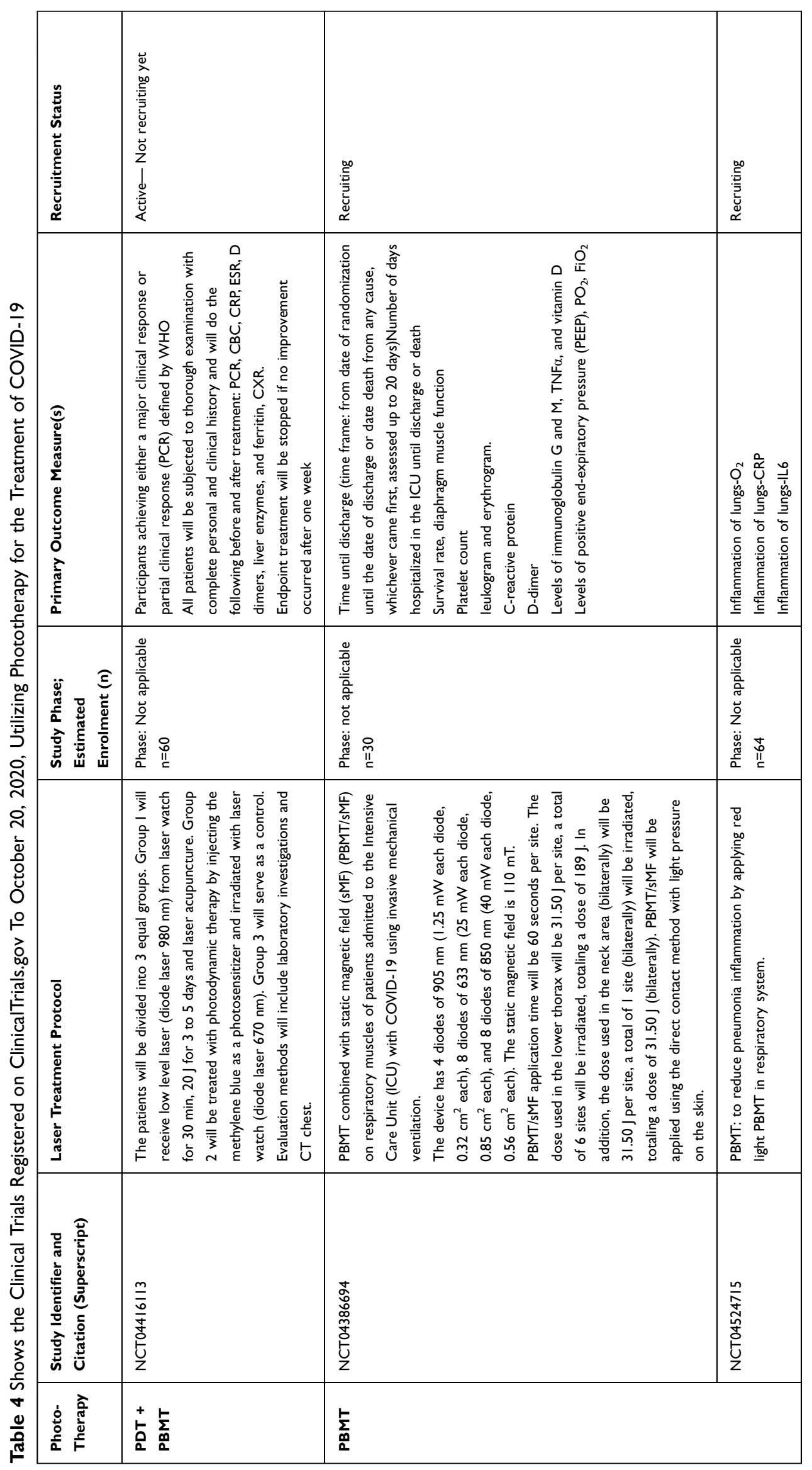




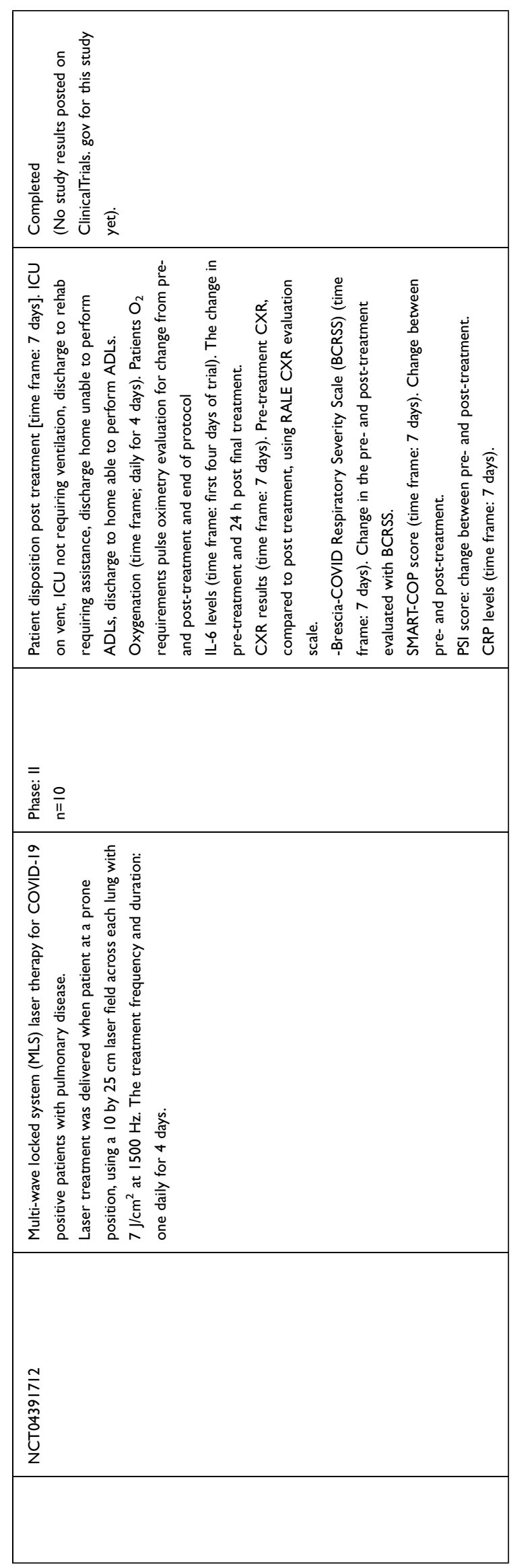


reported a substantial improvement on the communityacquired pneumonia assessment (CAPA) tool. Importantly it wasreported that the respiratory indices, radiological findings, oxygen requirements and patient outcomes have improved over several days of treatment without the need for a ventilator. This report has presented the significance of supportive PBMT in a patient with severe COVID-19 pneumonia. Future randomized controlled clinical trials with large data are warranted to evaluate PBMT effects on clinical outcomes in patients with COVID-19 pneumonia.

In the present review, we highlighted that patients with comorbidities can be subjected to a higher risk of complications when infected with SARS-CoV-2 virus. ${ }^{9-11}$ Obesity is one them. This is due to a low respiratory system compliance, increased inflammatory cytokines and an activated immune system secondary to excess adiposity. Another recent case report by Sigman et al, evaluated the effect of PBMT in treating a morbidly obese 32-year-old Asian female with severe COVID-19 $9^{211}$ who was irradiated with the same protocol of the previous case report. ${ }^{102}$ The utilized evaluation methods of PBMT outcomes are as follows: on CXR, pneumonia severity indices (SMART-COP and Brescia-COVID) and pneumonia severity indices blood inflammatory markers (IL-6, ferritin, and CRP), and oxygen requirements and saturation in a patient with severe COVID-19 and morbid obesity. The results have shown that $\mathrm{SpO}_{2}$ via pulse oximetry increased to $97-99 \%$ on 1-3 L oxygen after PBMT, compared to baseline where $\mathrm{SpO}_{2}$ via pulse oximetry was $88-93 \%$ on 5-6 L oxygen. Additionally, a reduction in the following values noted after PBMT: RALE score from eight to three, Brescia-COVID from four to zero, and SMART-COP from five to zero, IL-6 from 45.89 to $11.7 \mathrm{pg} / \mathrm{mL}$, ferritin from 359 to $175 \mathrm{ng} / \mathrm{mL}$ and CRP from 3.04 to $1.43 \mathrm{mg} / \mathrm{dL}$. This report suggests that adjunct PBMT can be safely combined with conventional treatment in patients with severe COVID-19 and morbid obesity. This coincides with a recent review by Mokmeli et al, who showed that PBMT combined with conventional medical therapy has the potential to prevent COVID-19 progress and improve symptoms. ${ }^{200}$

Given the abovementioned two cases, there is a relatively recent trend towards utilization of PBMT in minimizing complications with patients who are more vulnerable to COVID-19 than others. Hence, there is an urgent need for RCTs with large data to validate this promising noninvasive therapy.

\section{The Rationale for Use of PDT in COVID-19}

The most appropriate photosensitizer (PS) for PDT in the airway tract seems to be methylene blue (MB, phenothiazine derivative) for its good performance for safety in various clinical therapeutic uses. MB has two absorption peaks at $\lambda 635$ and $\lambda 670 \mathrm{~nm}$ and its complete absorption spectrum ranges from of $\lambda 609 \mathrm{~nm}$ to $\lambda 690 \mathrm{~nm}$, with a low slope trend of the ascending part of the absorption curve and a sudden drop of the descending part. ${ }^{212}$ The scientific literature clearly shows that both $\mathrm{MB}$ and riboflavin can be used to inactivate coronaviruses, using a photodynamic process, although the cellular mechanisms are not completely understood. It is assumed that the COVID-19 viruses accumulate the photosensitive molecules due to their energetic potential. Photodynamic excitation by an appropriately adapted light source (lasers or LEDs) leads to the formation of ROS and singlet $\mathrm{O}_{2}\left({ }^{1} \mathrm{O}_{2}\right)$, which destroys the cell membranes of the viruses. ${ }^{213,214}$ Knowing that most of the viruses have either DNA or RNA (single or double stranded) core and an outer protein cover or lipid, thereby, the basic structure of viruses contains three principal molecular targets for viral protein disulfide isomerase (PDI) and for the reaction with the generated ROS: nucleic acids, virus proteins, and if present, viral lipids. ${ }^{215}$ Those with viral lipids and/or protein envelope, in general seem to be more sensitive to using PDT/PDI (photodynamic inactivation) than those without. ${ }^{216}$ It is noteworthy that photodynamic damage is likely to occur very close to the intracellular location of the PS, which plays a crucial part in the apoptosis process, in conjunction with other factors such as the overall PDT dose (PS concentration $\dot{x}$ light fluence). ${ }^{217}$

Interestingly, SARS-CoV-2 has a number of distinguishing features including a protein envelope and lipids that would make it susceptible to treatment with PDT, ${ }^{218}$ as well as positive stranded RNA virus and belongs to the beta CoVs category. It has a round/elliptic and often pleomorphic form, and a diameter of approximately 60-140 nm. Like other CoVs, it is sensitive to ultraviolet rays and heat. ${ }^{218}$ As a result, safe and potent vaccine production faces many challenges, which might take a longer time to be on the market. Importantly, at the onset of the symptoms such as, fever, cough, and headaches, a significant number of viruses are bound to ACE2 receptors in the mucosa of the oral cavity, throat and nasal cavity, ${ }^{219}$ which allow easy access to PDT. Reduced viral load via PDT process can stimulate an immune reaction and the formation of protective antibodies, while favoring a mild or 
moderate course of disease without severe lung dysfunction or damage. ${ }^{220}$ It is noteworthy that the upper respiratory tract is the main harbor for opportunistic pathogen propagation. This is due to the natural colonization in the oropharynx. On this note the pharyngotonsillitis PDT can facilitate a reduction in these pathogens and act as a safeguard to prevent their penetration into the mucosal barriers. Hence, it is a good helper in the phagocytosis process, including inactivation of pathogen proliferation.213 This has been demonstrated by Blanco et al, reporting a clinical PDT protocol against pharyngotonsillitis in reducing more than $90 \%$ of the symptoms related to the disease after $24 \mathrm{~h}$ were observed. Hence, PDT is significant to inactivation the viral infection and reduce viral load in the respiratory tract. $^{221}$ Interestingly, many studies investigated the methods of delivery of the PS to the respiratory tract.

The most common vehicle for PS delivery for PDT of pulmonary diseases is via intravenous administration. This, however, is subjected to an intrinsic difficulty in targeting respiratory tract pathogen. ${ }^{222}$ As a result, nebulization can assist to overcome the lack of equal distribution of the PS and reduce adverse effects by delivering the PS directly to the lung. Fine catheter is another vehicle to deliver the light through the cricothyroid membrane. This technique could be useful in COVIS-19 patients with tracheostomy, thereby, MB-PDT in a topical setting is safe without expected morbidity. ${ }^{223}$ Given the current treatment protocols for COVID-19 management, MBmediated PDT could be a potential treatment modality for an early and advanced bronchopulmonary infection.

An in vivo animal study by Geralde et al, ${ }^{224}$ has shown the efficiency of PDT protocol in eradicating bacterial pneumonia by using an extracorporeal illumination via a custom-made laser device contained 18 clusters of laser diodes, emitting monochromatic light of $\lambda 780 \mathrm{~nm}$ at irradiance of $60 \mathrm{~mW} / \mathrm{cm}^{2}$ and a total dose of $120 \mathrm{~J} / \mathrm{cm}^{2}$ to activate nebulized indocyanine green (ICG) as PS. The ICG concentration of $100 \mu \mathrm{mol} / \mathrm{L}$ and $15 \mu \mathrm{L}$ was instilled in each nostril of the mouse. The number of recruited infected mice with Streptococcus pneumoniae cells was ten. The authors observed no deaths in the PDT group, compared to $60 \%$ in the control group. In addition, the colony forming unit recovery ranged from $10^{3}-10^{4} /$ mouse in the control group, whilst no bacteria recovered in $80 \%$ of the animals in the PDT group. Therefore, the authors concluded that clinical implementation of this protocol (extracorporeally-illuminated photodynamic inactivation of pneumonia) is a significant steping-stone toward PDT potential as a single therapy or as an adjunct to antibiotics treatment modality. Another in vivo animal study by Kassab et al, evaluated the viability of three photosensitizers (ICG, the chlorine photodithazine, and porphyrin photogem) in a jet nebulizer device, which was shown to be effective to target the lung directly. ${ }^{225}$

A randomized controlled trial/placebo study by Schikora et al, evaluated the effects of antimicrobial PDT (a-PDT) on following variables: SARS-CoV-2 viral load at early stages of the infection, viral load in the lung, inflammation and severe damage on the lung, clinical course of treatment and mortality rate. ${ }^{226}$ The following a-PDT protocol was utilized and applied on 300 participants who tested positive for COVID-19, whereas the placebo group $(n=330)$ received sham. MB ( $1 \%$ solution of methylthioninium-chloride dissolved in a $5 \%$ glucose solution) was applied to flush the oral cavity and throat and it was sprayed in the nostrils: $\lambda 660 \mathrm{~nm}$ photonic energy irradiation applied at power output of $240 \mathrm{~mW}$ for five minutes, at a fluence of $72 \mathrm{~J} / \mathrm{cm}^{2}$ (total fluence $360 \mathrm{~J} / \mathrm{cm}^{2}$ ). The PCR real time testing employed to determine the viral load pretreatment (baseline) and immediately after a-PDT. In addition, four weeks post a-PDT the patients were tested, using ELISA test to evaluate the formation of antibodies to SARS-CoV-2 virus. The results identified the following findings: (1) a reduction in mortality rate $(0.7$ in PDT and placebo group vs $3.3 \%$ in placebo group), (2) a significant reduction in the severity of the course of disease (2.6\% vs $19 \%$ ) and attenuation of COVID-19 progression (97\% vs 81\%) in PDT group. As a result, a reduction of viral load in throat, oral and nasal cavities revealed by PCR test immediately after each five-stage treatment cycle. Ultimately, no adverse effects were reported. In is important to mention that $\mathrm{MB}$ alone has no impact in reducing the viral load without photodynamic excitation with a light source. The authors concluded that a-PDT is an effective, innovative, accessible, cost-effective treatment modality in COVID-19 management without adverse effects. It is noteworthy that another significant application of PDT in COVID-19 pandemic is plasma inactivation of the SARS-CoV-2 to ensure safe blood transfusion. ${ }^{227}$

Several studies have reported successful treatments of coronaviruses by PDT, using riboflavin as PS. ${ }^{228,229}$ Riboflavin and UV light effectively reduced the titer of MERS-CoV in human plasma products, including the platelets to below the limit of detection, suggesting that the treatment process may reduce the risk of transfusion 
transmission of MERS-CoV. ${ }^{228,229}$ It is Important to note that a study employed $\lambda 405 \mathrm{~nm}$ light can have antimicrobial properties to eliminate the infection risk and can be propagated despite commonly known approaches in PDT and PDI. The design of personal mobile devices for the purpose of biological war can be proposed and used in such a simple form as mobile application, which program LED to irradiate specific wavelengths. Moreover, in terms of viruses inactivation, light therapy with a range of blue wavelengths between $\lambda 420 \mathrm{~nm}$ and $\lambda 430 \mathrm{~nm}$ can inactivate the leukemia virus. ${ }^{200}$ In this context, the visible light of the electromagnetic spectrum contributes into the PDT of the plasma to inactivate many viruses such as herpes simplex (HSV-1) and HIV. ${ }^{213,230,231}$ It is noteworthy that the recent reports have shown that blue light in a pulsed emission mode was 20-100 times more potent than in continuous emission mode to inactivate the opportunistic bacterial infections. ${ }^{232,233}$ This light source could be of great potential, as an antiviral agent to inactivate SARS$\mathrm{CoV}-2$, which requires further investigations.

Based on the abovementioned science and evidencedbased practice, phototherapy can be utilized as a single treatment modality or as an adjunct to the standard treatment protocol/s to inactivate SARS-CoV-2 virus, decrease its viral load, and reverse the cytokine storm, which can assist in modulating the host immune system.

\section{The Current Ongoing Phototherapy Registered RCTs for COVID-19 Management}

For all the abovementioned, phototherapy deserves great attention from researchers and clinicians for future longterm RCTs in the management of COVID-19. This coincides with a recent critical review by Hanna et al, showed that phototherapy is a promising treatment modality in COVID19 pandemic that demands to be validated by robust and rigorous randomized, double blind, placebo-controlled, clinical trials to evaluate its impartial outcomes and safety. ${ }^{234}$

Taking into consideration the abovementioned potential of phototherapy in COVID-19 management, at this stage only four RCTs have been registered at clinicaltrials.gov, which are either in the recruiting phase or not recruiting yet or completed phase without reported results (Table 4). These trials are highlighted below.

An ongoing RCT clinical trial (not recruiting yet stage) ${ }^{235}$ aims to evaluate the effects of PBMT and PDT, compared to control in treating COVID-19 patients, in order to achieve a major clinical response or partial respond defined by WHO in a time-frame of two weeks (Table 4). This trial is including hospitalized patients with positive COVID-19 with one or a combination of the following symptoms; fever, cough, shortness of breath, chills, muscle pain, a new loss of taste or smell, vomiting or diarrhea and/or sore throat, whilst it is excluding critical cases admitted to ICU, respiratory distress grade III and IV, as well patients on mechanical ventilator. Laboratory investigations (molecular-based assays; serology and antigen) and CT chest utilized to evaluate the trial clinical outcomes. The 60 recruited patients would be randomized as follows: group I irradiates with $\lambda 980 \mathrm{~nm}$ PBM from laser watch, which is applied on wrist on the radial artery for $30 \mathrm{~min}, 20 \mathrm{~J}$ for 3-5 days and laser acupuncture trigger points; group 2 receives PDT according to the following protocol, $670 \mathrm{~nm}$ laser watch, methylene blue (MB) as a photosensitizer (PS) of 0.1 to $0.2 \mathrm{~mL}$ (1\% solution per kilogram of body weight) injected intravenously very slowly over a period of several minutes, after one hour, an application of light photonic dose: 100-200 J/ $\mathrm{cm}^{2}$, $50-100 \mathrm{~mW} / \mathrm{cm}^{2}\left(50 \mathrm{~mW} / \mathrm{cm}^{2}\right.$ increased the phototoxic response as well as the fractionated light application) twice per week; whilst group 3 serves as a control (conventional treatment protocol).

In terms of PBMT, there are two ongoing RCTs that are in the recruiting stage (Table 4). ${ }^{236,237}$ One of these RCTs aims to evaluate the effects of LED PBMT on lung inflammation in COVID-19 patients. $^{237}$ Sixty-four participants above 18 -yearsold diagnosed with COVID-19 and exhibiting moderate-acute respiratory distress were recruited, whilst participants with photosensitive condition or medication, active chemotherapy treatment or other cancer treatment, autoimmune disorders, as well pregnant or possibly pregnant or planning pregnancy prior to the end of study participation would be excluded. In this trial, LED light source in the red and IR range of PBMT is utilized. All participants continue receiving standard treatments for COVID-19 symptoms. This trial aims to determine if a reduction of pneumonic inflammation occurs after PBMT, applying red-light LED source on the respiratory system of COVID-19 patients, suffering from acute viral pneumonia. The protocol of PBMT was not recorded. The following methods of assessment of outcomes are SpO2, CRP, and IL-6 levels. Alternatively, a randomized, triple-blind, placebo-controlled trial is registered (not recruiting yet) ${ }^{237}$ to investigate the effects LEDs PBMT combined with static magnetic field (sMF) (LEDs PBMT/sMF) on respiratory muscles of patients admitted to the ICU with COVID-19, using invasive mechanical ventilation. The laser protocol described in Table 1 shows that a clustered probe of eight diodes of $\lambda 850 \mathrm{~nm}$, eight diodes 
of $\lambda 633 \mathrm{~nm}$ and four diodes of $\lambda 905 \mathrm{~nm}$. This indicates that the light would interact with tissue at various depth of penetration to achieve optimal outcome. Various variables would be assessed (Table 4) to determine whether this combined therapy has an influence in minimizing the impact of COVID-19 on the respiratory muscles, accelerating the ventilatory weaning process and optimizing the functional capacity of the involved muscles.

One completed $\mathrm{RCT}^{238}$ evaluated the effectiveness of multiwave locked system (MLS) laser therapy, as a treatment for pulmonary complications due to COVID-19 infection. Laser treatment was delivered with patients in a prone position, using a 10 by $25 \mathrm{~cm}$ laser field across each lung with $7 \mathrm{~J} / \mathrm{cm}^{2}$ at $1500 \mathrm{~Hz}$. The treatment frequency and duration: once daily for four days (Table 4). However, no study results are posted on clinicaltrials.gov yet.

It is noteworthy that despite the scientific evidence-based research consistently suggesting the potential of PDT in COVID-19 which is intensively emphasized in our review, only one RCT to date is registered to evaluate the effects of PDT combined with PBMT in COVID-19 management. Further studies are warranted to confirm the study design of an ideal photodynamic protocol. Moreover, the authors of the present review observed that phototherapy protocols for each stage (early and medium-severe) of COVID-19 are warranted to justify therapeutic outcomes and ensure safety.

\section{Conclusion}

This review has produced a high quality evidence, which can be utilized by the clinical and the scientific community for future reference, as the knowledge and understanding the virus SARS-CoV-2 are evolving, in terms of its epidemiological and clinical manifestations, which ultimately map the strategic path toward an effective and safe treatment and production of potent and safe vaccine. To date, there is neither a reliable safe vaccine nor an effective safe treatment modality, specifically designed to treat COVID-19. There are novel therapeutic innovations such as immunotherapy and cellular therapy, which require further investigation. Phototherapy is a welldocumented treatment modality, which can play a crucial role in COVID-19 management. However, further clinical research is mandatory in this regard.

\section{Funding}

This work was funded by National Research Development Projects to finance excellence (PFE)-37/2018- 2020 granted by the Romanian Ministry of Research and Innovation.

\section{Disclosure}

The authors have declared no conflicts of interest in this work.

\section{References}

1. Available from: https://www.ecdc.europa.eu/en/geographical-dis tribution-2019-ncov-cases. Accessed October 20, 2020.

2. Lin L, Lu LF, Cao W, et al. Hypothesis for potential pathogenesis of SARS-CoV-2 infection-a review of immune changes in patients with viral pneumonia. Emerg Microb Infect. 2020;9 (1):727-732. doi:10.1080/22221751.2020.1746199

3. Mason RJ. Pathogenesis of COVID-19 from a cell biology perspective. Eur Respir J. 2020;55(4):2000607. doi:10.1183/ 13993003.00607-2020

4. $\mathrm{Wu} \mathrm{Z,} \mathrm{McGoogan} \mathrm{JM.} \mathrm{Characteristics} \mathrm{of} \mathrm{and} \mathrm{important} \mathrm{lessons}$ from the coronavirus disease 2019 (COVID-19) outbreak in China: summary of a report of 72314 cases from the Chinese center for disease control and prevention [published online ahead of print, 2020 Feb 24]. JAMA. 2020;10.1001/jama.2020.2648. doi:10.1001/jama.2020.2648

5. Wrapp D, Wang N, Corbett KS, et al. Cryo- EM structure of the 2019-nCoV spike in the prefusion conformation. Science. 2020;367(6483):1260-1263. doi:10.1126/science.abb2507

6. Li F. Structure, function, and evolution of coronavirus spike proteins. Annu Rev Virol. 2016;3(1):237-261. doi:10.1146/ annurev-virology-110615-042301

7. Gui M, Song W, Zhou H, et al. Cryo-electron microscopy structures of the SARS-CoV spike glycoprotein reveal a prerequisite conformational state for receptor binding. Cell Res. 2017;27 (1):119-129. doi:10.1038/cr.2016.152

8. Walls AC, Xiong X, Park YJ, et al. Unexpected receptor functional mimicry elucidates activation of coronavirus fusion. Cell. 2019;176(5):1026-39.e15. doi:10.1016/j.cell.2018.12.028

9. Huang C, Wang Y, Li X, et al. Clinical features of patients infected with 2019 novel coronavirus in Wuhan, China. Lancet. 2020;395(10223):497-506. doi:10.1016/S0140-6736(20)30183-5

10. Chen N, Zhou M, Dong X, et al. Epidemiological and clinical characteristics of 99 cases of 2019 novel coronavirus pneumonia in Wuhan, China: a descriptive study. Lancet. 2020;395 (10223):507-513. doi:10.1016/S0140-6736(20)30211-7

11. Available from: https://coronavirus.jhu.edu/data/racial-data-trans parency. Accessed October 20, 2020.

12. Available from: https://clinicaltrials.gov/ct2/results?cond= COVID-19. Accessed October 20, 2020.

13. Mackenzie JS, Smith DW. COVID-19: a novel zoonotic disease caused by a coronavirus from China: what we know and what we don't. Microbiol Aust. 2020;MA20013. doi:10.1071/MA20013

14. Zhou P, Yang XL, Wang XG, et al. A pneumonia outbreak associated with a new coronavirus of probable bat origin. Nature. 2020;579 (7798):270-273. doi:10.1038/s41586-020-2012-7

15. Cascella M, Rajnik M, Cuomo A, et al. Features, evaluation, and treatment of coronavirus (COVID-19). In: StatPearls. Treasure Island (FL): StatPearls Publishing; 2020.

16. Lam TT, Shum MH, Zhu HC, et al. Identifying SARS-CoV-2 related coronaviruses in Malayan pangolins. Nature. 2020;583 (7815):282-285. doi:10.1038/s41586-020-2169-0

17. Jin $\mathrm{Y}$, Yang H, Ji W, et al. Virology, epidemiology, pathogenesis, and control of COVID-19. Viruses. 2020;12(4):372. doi:10.3390/ v12040372

18. van Doremalen N, Bushmaker T, Morris DH. Aerosol and surface stability of SARS-CoV-2 as compared with SARS-CoV-1. $N$ Engl J Med. 2020;382(16):1564-1567. doi:10.1056/ NEJMc2004973 
19. Salehi S, Abedi A, Balakrishnan S, et al. Coronavirus disease 2019 (COVID- 19): a systematic review of imaging findings in 919 patients. AJR Am J Roentgenol. 2020:1-7. doi:10.2214/AJR.20.23034.

20. Hamming I, Timens W, Bulthuis ML, et al. Tissue distribution of ACE2 protein, the functional receptor for SARS coronavirus. A first step in understanding SARS pathogenesis. $J$ Pathol. 2004;203:631-637. doi:10.1002/path.1570

21. Li H, Liu SM, Yu XH, et al. Coronavirus disease 2019 (COVID19): current status and future perspectives. Int $J$ Antimicrobial Agents. 2020;55(5):105951. doi:10.1016/j.ijantimicag.2020.1

22. Available from: https://www.cdc.gov/coronavirus/2019-ncov/hcp/clini cal-guidance-management-patients.html. Accessed October 20, 2020.

23. Thevarajan I, Buising KL, Cowie BC. Clinical presentation and management of COVID-19. Med J Aust. 2020;213(3):134-139. doi: $10.5694 / \mathrm{mja} 2.50698$

24. Ho JC, Chan KN, Hu WH, et al. The effect of aging on nasal mucociliary clearance, beat frequency, and ultrastructure of respiratory cilia. Am J Respir Crit Care Med. 2001;163:983988. doi:10.1164/ajrccm.163.4.9909121

25. Epidemiology Working Group for NCIP Epidemic Response. Chinese center for disease control and prevention. Zhonghua Liu Xing Bing Xue Za Zhi. 2020;41(2):145-151. doi:10.3760/ cma.j.issn.0254-6450.2020.02.003.

26. Di Stadio A, Ricci G, Greco A, et al. Mortality rate and gender differences in COVID-19 patients dying in Italy: a comparison with other countries. Eur Rev Med Pharmacol Sci. 2020;24 (8):4066-4067. doi:10.26355/eurrev-202004-20980

27. Jin JM, Bai P, He W, et al. Gender differences in patients with COVID-19: focus on severity and mortality. Front Public Health. 2020;8:152. doi:10.3389/fpubh.2020.00152

28. Chen H, Guo J, Wang C, et al. Clinical characteristics and intrauterine vertical transmission potential of COVID-19 infection in nine pregnant women: a retrospective review of medical records. Lancet. 2020;395(10226):809-815. doi:10.1016/S01406736(20)30360-3

29. Jones VG, Mills M, Suarez D, et al. COVID-19 and Kawasaki disease: novel virus and novel case. Hosp Pediatr. 2020;10 (6):537-540. doi:10.1542/hpeds.2020-0123

30. Esper FED, Shapiro C, Weibel D, et al. Association between a novel human coronavirus and Kawasaki disease. J Infect Dis. 2005;191(4):499-502. doi:10.1086/428291

31. Available from: https://www.cdc.gov/coronavirus/2019-ncov/ need-extra-precautions/index.html. Accessed October 20, 2020.

32. Curtis N, Sparrow A, Ghebreyesus TA, et al. Considering BCG vaccination to reduce the impact of COVID-19. Lancet. 2020;395 (10236):1545-1546. doi:10.1016/S0140-6736(20)31025-4

33. Lu CW, Liu XF, Jia ZF. 2019-nCoV transmission through the ocular surface must not be ignored. Lancet. 2020;395(10224):e39. doi:10.1016/S0140-6736(20)30313-5

34. Xia J, Tong J, Liu M, et al. Evaluation of coronavirus in tears and conjunctival secretions of patients with SARS-CoV-2 infection. $J$ Med Virol. 2020;92(6):589-594. doi:10.1002/jmv.25725

35. Lauer SA, Grantz KH, Bi Q, et al. The incubation period of coronavirus disease 2019 (COVID-19) from publicly reported confirmed cases: estimation and application. Ann Intern Med. 2020;172(9):577-582. doi:10.7326/M20-0504

36. Novel coronavirus (2019-ncov) situation report-7. World Health Organization (WHO). 2020 January 27. Accessed October 20, 2020.

37. China's national health commission news conference on coronavirus. Al Jazeera. 2020 January 26. Accessed October 20, 2020.

38. Symptoms of novel coronavirus (2019-nCoV)-CDC. Accessed October 20, 2020.

39. Varia M, Wilson S, Sarwal S, et al. Investigation of a nosocomial outbreak of severe acute respiratory syndrome (SARS) in Toronto, Canada. CMAJ. 2003;169:285-292.
40. Rothe C, Schunk M, Sothmann P, et al. Transmission of 2019nCoV infection from an asymptomatic contact in Germany. $N$ Engl J Med. 2020;382(10):970-971. doi:10.1056/NEJMc2001468

41. Quilty BJ, Clifford S, Flasche S, et al. Effectiveness of airport screening at detecting travellers infected with novel coronavirus (2019-nCoV). Euro Surveill. 2020;25(5):2000080. doi:10.2807/ 1560-7917.ES.2020.25.5.2000080

42. Guan W, Ni Z, Hu Y, et al. Clinical characteristics of 2019 novel coronavirus infection in China. N Engl J Med J Med. 2020;382 (18):1708-1720. doi:10.1056/NEJMoa2002032

43. Coronavirus incubation could be as long as 27 days, Chinese provincial government says. Reuters. 2020 Feb 22. Accessed October 20, 2020.

44. Xu Z, Shi L, Wang Y, et al. Pathological findings of COVID-19 associated with acute respiratory distress syndrome. Lancet Respir Med. 2020;8(4):420-422. doi:10.1016/S2213-2600(20) 30076-X

45. Ding Y, Wang H, Shen $\mathrm{H}$, et al. The clinical pathology of severe acute respiratory syndrome (SARS): a report from China. $J$ Pathol. 2003;200(3):282-289. doi:10.1002/path.1440

46. Deshmukh V, Motwani R, Kumar A, et al. Histopathological observations in COVID-19: a systematic review. J Clin Pathol. 2020:jclinpath-2020-206995. doi:10.1136/jclinpath-2020206995.

47. Chung M, Bernheim A, Mei X, et al. CT imaging features of 2019 novel coronavirus (2019-nCoV). Radiology. 2020;295(1):202207. doi:10.1148/radiol.2020200230

48. Li W, Cui H, Li K, et al. Chest computed tomography in children with COVID-19 respiratory infection. Pediatr Radiol. 2020;50 (6):796-799. doi:10.1007/s00247-020-04656-7

49. Corman VM, Landt O, Kaiser M, et al. Detection of 2019 novel coronavirus (2019-nCoV) by real-time RT-PCR. Euro Surveill. 2020;25(3):2000045. doi:10.2807/1560-7917

50. Chan JF, Yuan S, Kok KH, et al. A familial cluster of pneumonia associated with the 2019 novel coronavirus indicating person-toperson transmission: a study of a family cluster. Lancet. 2020;395 (10223):514-523. doi:10.1016/S0140-6736(20)30154-9

51. Carver C, Jones N. Comparative accuracy of oropharyngeal and nasopharyngeal swabs for diagnosis of COVID-19. Oxford COVID-19 evidence service team centre for evidence-based medicine; 2020. Available from: https://www.cebm.net/covid-19/com parative-accuracy-of-oropharyngeal-and-nasopharyngeal-swabsfor-diagnosis-of-covid-19/. Accessed October 20, 2020.

52. Wang W, Xu Y, Gao R, et al. Detection of SARS-CoV-2 in different types of clinical specimens. JAMA. 2020;323 (18):1843-1844. doi:10.1001/jama.2020.3786

53. To KK, Tsang OT, Yip CC, et al. Consistent detection of 2019 novel coronavirus in saliva. Clin Infect Dis. 2020;71(15):841843. doi:10.1093/cid/ciaa149

54. Lipsitch M, Kahn R, Mina MJ. Antibody testing will enhance the power and accuracy of COVID-19 prevention trials. Nat Med. 2020;26(6):818-819. doi:10.1038/s41591-020-0887-3

55. Zhang F, Abudayyeh OO, Gootenberg JS. A Protocol for Detection of COVID-19 Using CRISPR Diagnostics. Cambridge, MA: Broad Institute, MIT; 2020.

56. Bosch BJ, van der Zee R, de Haan CA, et al. The coronavirus spike protein is a class I virus fusion protein: structural and functional characterization of the fusion core complex. J Virol. 2003;77 (16):8801-8811. doi:10.1128/jvi.77.16.8801-8811.2003

57. Yuan Y, Cao D, Zhang Y, et al. Cryo-EM structures of MERS$\mathrm{CoV}$ and SARS-CoV spike glycoproteins reveal the dynamic receptor binding domains. Nat Commun. 2017;8(1):15092. doi:10.1038/ncomms 15092

58. Hoffmann M, Kleine-Weber H, Schroeder S. SARS-CoV-2 cell entry depends on ACE2 and TMPRSS2 and is blocked by a clinically proven protease inhibitor. Cell. 2020;181(2):271-280. doi:10.1016/j.cell.2020.02.052 
59. Braciale TJ, Hahn YS. Immunity to viruses. Immunol Rev. 2013;255(1):5-12. doi:10.1111/imr.12109

60. Li W, Moore MJ, Vasilieva N, et al. Angiotensin-converting enzyme 2 is a functional receptor for the SARS coronavirus. Nature. 2003;426(6965):450-454. doi:10.1038/nature02145

61. Zou X, Chen K, Zou J, et al. Single-cell RNA-seq data analysis on the receptor ACE2 expression reveals the potential risk of different human organs vulnerable to $2019-\mathrm{nCoV}$ infection. Front Med. 2020;14(2):185-192. doi:10.1007/s11684-020-0754-0

62. Sungnak W, Huang N, Bécavin C, et al. HCA lung biological network. SARS-CoV-2 entry factors are highly expressed in nasal epithelial cells together with innate immune genes. Nat Med. 2020;26(5):681-687. doi:10.1038/s41591-020-0868-6

63. Das SK. The pathophysiology, diagnosis and treatment of corona virus disease 2019 (COVID-19). Indian J Clin Biochem. 2020;35 (4):1-12. doi:10.1007/s12291-020-00919-0

64. Wiersinga WJ, Rhodes A, Cheng AC, et al. Pathophysiology, transmission, diagnosis, and treatment of coronavirus disease 2019 (COVID-19): a review. JAMA. 2020;324(8):782-793. doi:10.1001/jama.2020.12839

65. Mancia G, Rea F, Ludergnani M, et al. Renin-angiotensin-aldosterone system blockers and the risk of COVID-19. $N$ Engl J Med. 2020;382(25):2431-2440. doi:10.1056/NEJMoa2006923

66. Fosbøl EL, Butt JH, Østergaard L, et al. Association of angiotensin-converting enzyme inhibitor or angiotensin receptor blocker use with COVID-19 diagnosis and mortality. JAMA. 2020;324 (2):168-77. doi:10.1001/jama.2020.11301

67. Ragab D, Salah Eldin H, Taeimah M, Khattab R, Salem R. The COVID-19 cytokine storm; what we know so far. Front Immunol. 2020;11:1446. doi:10.3389/fimmu.2020.01446

68. Shimizu M. Clinical features of cytokine storm syndrome. In: Cron R, Behrens E, editors. Cytokine Storm Syndrome. Cham: Springer; 2019:31-42. doi:10.1007/978-3-030-22094-5 3

69. Fletcher-Sandersjöö A, Bellander BM. Is COVID-19 associated thrombosis caused by overactivation of the complement cascade? A literature review [published online ahead of print, 2020 Jun 18]. Thromb Res. 2020;194:36-41. doi:10.1016/j. thromres.2020.06.027

70. Klok FA, Kruip MJHA, van der Meer NJM, et al. Incidence of thrombotic complications in critically ill ICU patients with COVID-19. Thromb Res. 2020;191:145-147. doi:10.1016/j. thromres.2020.04.013

71. Lai C, Shih T, Ko W, Tang H, Hsueh P-R. Severe acute respiratory syndrome coronavirus 2 (SARS-CoV-2) and coronavirus disease-2019 (COVID-19): the epidemic and the challenges. Int J Antimicrob Agents. 2020;55(3):105924. doi:10.1016/j.ijantimicag.2020.105924

72. Ishikawa T. Clinical preparedness for cytokine storm induced by the highly pathogenic H5N1 influenza virus. J Pharmacogenom Pharmacoproteomics. 2012;3(6):1000e131. doi:10.4172/2153$0645.1000 \mathrm{e} 131$

73. Task Force BM, BASE Medicine Task Force. COVID-19: facts and recommendations from A to Z. Sci Insight. 2020;33(1):138158. doi:10.15354/si.20.re061

74. Yousefifard M, Zali A, Mohamed Ali K, et al. Antiviral therapy in management of COVID-19: a systematic review on current evidence. Arch Acad Emerg Med. 2020;8(1):e45.

75. Scagnolari C, Vicenzi E, Bellomi F, et al. Increased sensitivity of SARS-coronavirus to a combination of human type I and type II interferons. Antivir Ther. 2004;9:1003-1011.

76. Stockman LJ, Bellamy R, Garner P. SARS: systematic review of treatment effects. PLoS Med. 2006;3(9):e343. doi:10.1371/journal.pmed.0030343

77. Chan JF, Yao Y, Yeung ML, et al. Treatment with lopinavir/ ritonavir or interferon- $\beta 1 \mathrm{~b}$ improves outcome of MERS-CoV infection in a nonhuman primate model of common marmoset. $J$ Infect Dis. 2015;212(12):1904-1913. doi:10.1093/infdis/jiv392
78. Chen F, Chan KH, Jiang Y, et al. In vitro susceptibility of 10 clinical isolates of SARS coronavirus to selected antiviral compounds. $J$ Clin Virol. 2004;31(1):69-75. doi:10.1016/j. jcv.2004.03.003

79. Loutfy MR, Blatt LM, Siminovitch KA, et al. Interferon alfacon-1 plus corticosteroids in severe acute respiratory syndrome: a preliminary study. JAMA. 2003;290(24):3222-3228. doi:10.1001/ jama.290.24.3222

80. Jr SB, Mossel EC, Peters CJ, et al. Interferon-beta and interferongamma synergistically inhibit the replication of severe acute respiratory syndrome-associated coronavirus (SARS-CoV). Virology. 2004;329(1):11-17. doi:10.1016/j.virol.2004.08.011

81. Zhou Q, Chen V, Shannon CP, et al. Interferon- $\alpha 2 b$ treatment for COVID-19. Front Immunol. 2020;11:1061. doi:10.3389/ fimmu.2020.01061

82. Available from: https://www.covid19treatmentguidelines.nih.gov/ immune-basedtherapy/immunomodulators/interferons/. Accessed October 20, 2020.

83. Adaptive COVID-19 treatment trial 3 (ACTT-3). Available from: https://clinicaltrials.gov/ct2/show/NCT04492475?term= NCT04492475\&draw=2\&rank=1. Accessed October 20, 2020.

84. Pegylated interferon lambda treatment for COVID-19. Available from: https:/clinicaltrials.gov/ct2/show/NCT04343976?term= NCT04343976\&draw=2\&rank=1. Accessed October 20, 2020.

85. An investigation into beneficial effects of interferon beta $1 \mathrm{a}$, compared to interferon beta $1 \mathrm{~b}$ and the base therapeutic regiment in moderate to severe COVID-19: a randomized clinical trial (COVIFERON). Available from: https://clinicaltrials.gov/ct2/ show $/ \mathrm{NCT} 04343768$ ?term $=$ NCT04343768\&draw $=2 \&$ rank $=1$. Accessed October 20, 2020.

86. LiverTox. Clinical and Research Information on Drug-Induced Liver Injury. Bethesda (MD): National Institute of Diabetes and Digestive and Kidney Diseases; 2012.

87. Wenzel RP, Edmond MB. Managing SARS amidst uncertainty. $N$ Engl J Med. 2003;348(20):1947-1948. doi:10.1056/ NEJMp030072

88. Morgenstern B, Michaelis M, Baer PC, et al. Ribavirin and interferon-beta synergistically inhibit SARS-associated coronavirus replication in animal and human cell lines. Biochem Biophys Res Commun. 2005;326(4):905-908. doi:10.1016/j. bbrc.2004.11.128

89. Khalili JS, Zhu H, Mak NSA, et al. Novel coronavirus treatment with ribavirin: groundwork for evaluation concerning COVID-19. J Med Virol. 2020;92(7):740-746. doi:10.1002/jmv.25798

90. de Wit E, Feldmann F, Cronin J, et al. Prophylactic and therapeutic remdesivir (GS-5734) treatment in the rhesus macaque model of MERS-CoV infection. Proc Natl Acad Sci. 2020;117 (12):6771-6776. doi:10.1073/pnas.1922083117

91. Wang M, Cao R, Zhang L, et al. Remdesivir and chloroquine effectively inhibit the recently emerged novel coronavirus (2019$\mathrm{nCoV}$ ) in vitro. Cell Res. 2020;30(3):269-271. doi:10.1038/ s41422-020-0282-0

92. Holshue ML, DeBolt C, Lindquist S, et al. First case of 2019 novel coronavirus in the United States. N Engl J Med. 2020;382 (10):929-936. doi:10.1056/NEJMoa2001191

93. Beigel JH, Tomashek KM, Dodd LE, et al. Remdesivir for the treatment of covid-19 - final report [published online ahead of print, 2020 Oct 8]. $N$ Engl J Med. 2020:NEJMoa2007764. doi:10.1056/NEJMoa2007764.

94. Wang Y, Zhang D, Du G, et al. Remdesivir in adults with severe COVID-19: a randomised, double-blind, placebo-controlled, multicentre trial. Lancet. 2020;395(10236):1569-1578. doi:10.1016/ S0140-6736(20)31022-9

95. Adaptive COVID-19 treatment trial (ACTT). Available from: https://clinicaltrials.gov/ct2/show/NCT04280705?term= NCT04280705\&draw=2\&rank=1. Accessed October 20, 2020. 
96. Study to evaluate the safety and antiviral activity of remdesivir (GS-5734TM) in participants with severe coronavirus disease (COVID-19). Available from: https://clinicaltrials.gov/ct2/show/ NCT04292899?term=NCT04292899\&draw=2\&rank=1.

Accessed October 20, 2020.

97. Study to evaluate the safety and antiviral activity of remdesivir (GS-5734 ${ }^{\mathrm{TM}}$ ) in participants with moderate coronavirus disease (COVID-19) compared to standard of care treatment. Available from: https://clinicaltrials.gov/ct2/show/NCT04292730?term= NCT04292730\&draw=2\&rank=1. Accessed October 20, 2020.

98. A study to evaluate the efficacy and safety of remdesivir plus tocilizumab compared with remdesivir plus placebo in hospitalized participants with severe COVID-19 pneumonia (REMDACTA). Available from: https://clinicaltrials.gov/ct2/ show/NCT04409262?term=NCT04409262\&draw=2\&rank=1. Accessed October 20, 2020.

99. Lv Z, Chu Y, Wang Y. HIV protease inhibitors: a review of molecular selectivity and toxicity. HIV AIDS (Auckl). 2015;7:95-104. doi:10.2147/HIV.S79956

100. Kim UJ, Won EJ, Kee SJ, et al. Combination therapy with lopinavir/ritonavir, ribavirin and interferon-alpha for Middle East respiratory syndrome. Antivir Ther. 2016;21(5):455-459. doi:10.3851/IMP3002

101. Lin S, Shen R, Guo X. Molecular modeling evaluation of the binding abilities of ritonavir and lopinavir to Wuhan pneumonia coronavirus proteases. bioRxiv. 2020. doi:10.1101/2020.01.31.929695

102. Yamamoto N, Yang R, Yoshinaka Y, et al. HIV protease inhibitor nelfinavir inhibits replication of SARS-associated coronavirus. Biochem Biophys Res Commun. 2004;318(3):719-725. doi:10.1016/j.bbrc.2004.04.083

103. Yamamoto N, Matsuyama S, Hoshino T, et al. Nelfinavir inhibits replication of severe acute respiratory syndrome coronavirus 2 in vitro. bioRxiv. 2020. doi:10.1101/2020.04.06.026476

104. Randomised evaluation of COVID-19 therapy (recovery). Available from: https://clinicaltrials.gov/ct2/show/ NCT04381936?term=NCT04381936\&draw $=2 \&$ rank $=1$. Accessed October 20, 2020

105. Khamitov RA, Loginova S, Shchukina VN, et al. Antiviral activity of arbidol and its derivatives against the pathogen of severe acute respiratory syndrome in the cell cultures. Vopr Virusol. 2008;53:9-13.

106. Huang L, Zhang L, Liu Y, et al. Arbidol for preventing and treating influenza in adults and children. Cochrane Database Syst Rev. 2017;2017(2):CD011489. doi:10.1002/14651858.CD011489.pub2

107. Zhang J, Zhou L, Yang Y, et al. Therapeutic and triage strategies for 2019 novel coronavirus disease in fever clinics. Lancet Respir Med. 2020;8(3):e11-e12. doi:10.1016/S2213-2600(20)30071-0

108. Zhu Z, Lu Z, Xu T, et al. Arbidol monotherapy is superior to lopinavir/ritonavir in treating COVID-19. J Infect. 2020;81(1): e21-e23. doi:10.1016/j.jinf.2020.03.060

109. Clinical study of arbidol hydrochloride tablets in the treatment of pneumonia caused by novel coronavirus. Available from: https:// clinicaltrials.gov/ct2/show/record/NCT04260594?term=arbidol\& cond $=$ covid $+19 \& d r a w=2 \&$ rank=3. Accessed October 20, 2020.

110. Savarino A, Boelaert JR, Cassone A, et al. Effects of chloroquine on viral infections: an old drug against today's diseases? Lancet Infect Dis. 2003;3(11):722-727. doi:10.1016/s1473-3099(03)00806-5

111. Yan Y, Zou Z, Sun Y, et al. Anti-malaria drug chloroquine is highly effective in treating avian influenza A H5N1 virus infection in an animal model. Cell Res. 2013;23(2):300-302. doi: $10.1038 /$ cr.2012.165

112. Cortegiani A, Ingoglia G, Ippolito M, et al. A systematic review on the efficacy and safety of chloroquine for the treatment of COVID-19. J Crit Care. 2020;57:279-283. doi:10.1016/j. jerc.2020.03.005
113. Dexamethasone treatment for severe acute respiratory distress syndrome induced by COVID-19 (DHYSCO). Available from: https://clinicaltrials.gov/ct2/show/NCT04347980?term= NCT04347980\&draw=2\&rank=1. Accessed October 20, 2020.

114. Randomized clinical trial for the prevention of sars-cov-2 infection (COVID-19) in healthcare personnel (EPICOS). Available from: https://clinicaltrials.gov/ct2/show/NCT04334928?term= NCT04334928\&draw=2\&rank=1. Accessed October 20, 2020.

115. Available from: http://chemocare.com/chemotherapy/drug-info/ dexamethasone.aspx. Accessed October 20, 2020.

116. Available from: https://www.nice.org.uk/guidance/ng159/ resources/covid19-prescribing-briefing-corticosteroids-pdf8839913581. Accessed October 20, 2020.

117. Mahase E. Covid-19: hydrocortisone can be used as alternative to dexamethasone, review finds. BMJ. 2020;370:m3472. doi:10.1136/bmj.m3472

118. Randomized clinical trial of intranasal dexamethasone as an adjuvant in patients with COVID-19. Available from: https:/clinical trials.gov/ct $2 /$ show $/$ NCT04513184?term $=$ NCT04513184\&draw $=$ 2\&rank=1. Accessed October 20, 2020.

119. Higher vs. lower doses of dexamethasone for COVID-19 and severe hypoxia (COVIDSTEROID2). Available from: https://clin icaltrials.gov/ct $2 / \mathrm{show} / \mathrm{NCT} 04509973$ ? term= NCT04509973\&draw=2\&rank=1. Accessed October 20, 2020.

120. Available from: https:/www.popsci.com/story/health/convales cent-plasma-covid-19-coronavirus/. Accessed October 20, 2020.

121. Pandey S, Vyas GN. Adverse effects of plasma transfusion. Transfusion. 2012;52(Suppl 1):65S-79S. doi:10.1111/j.15372995.2012.03663.x

122. Zhou B, Zhong N, Guan Y. Treatment with convalescent plasma for influenza A (H5N1) infection. $N$ Engl J Med. 2007;357 (14):1450-1451. doi:10.1056/NEJMc070359

123. van Griensven J, Edwards T, de Lamballerie X, et al. Evaluation of convalescent plasma for Ebola virus disease in Guinea. $N$ Engl $J$ Med. 2016;374(1):33-42. doi:10.1056/NEJMoa1511812

124. Cheng Y, Wong R, Soo YO, et al. Use of convalescent plasma therapy in SARS patients in Hong Kong. Eur J Clin Microbiol Infect Dis. 2005;24(1):44-46. doi:10.1007/s10096-004-1271-9

125. Duan K, Liu B, Li C, et al. Effectiveness of convalescent plasma therapy in severe COVID-19 patients. Proc Natl Acad Sci USA. 2020;117(17):9490. doi:10.1073/pnas.2004168117:202004168

126. Wang C, Li W, Drabek D, et al. A human monoclonal antibody blocking SARS-CoV-2 infection. Nat Commun. 2020;11(2251):16. doi:10.1038/s41467-020-16256-y

127. Guaraldi G, Meschiari M, Cozzi-Lepri A, et al. Tocilizumab in patients with severe COVID-19: a retrospective cohort study [published correction appears in lancet rheumatol. 2020;2(10): e591]. Lancet Rheumatol. 2020;2(8):e474-e484. doi:10.1016/ S2665-9913(20)30173-9

128. Available from: https://www.versusarthritis.org/about-arthritis/ treatments/drugs/tocilizumab/. Accessed October 20, 2020.

129. Sheppard M, Laskou F, Stapleton PP, et al. Tocilizumab (Actemra). Hum Vaccin Immunother. 2017;13(9):1972-1988. doi:10.1080/ 21645515.2017.1316909

130. Lan SH, Lai CC, Huang HT, et al. Tocilizumab for severe COVID-19: a systematic review and meta-analysis. Int $J$ Antimicrob Agents. 2020;56(3):106103. doi:10.1016/j. ijantimicag.2020.106103

131. Available from: https://www.drugs.com/kineret.html. Accessed October 20, 2020.

132. Huet T, Beaussier H, Voisin O, et al. Anakinra for severe forms of COVID-19: a cohort study. Lancet Rheumatol. 2020;2(7):e393e400. doi:10.1016/S2665-9913(20)30164-8

133. Favipiravir combined with tocilizumab in the treatment of corona virus disease; 2019. Available from: https:/clinicaltrials.gov/ct2/ show/NCT04310228. Accessed October 20, 2020. 
134. A study to evaluate the safety and efficacy of tocilizumab in patients with severe COVID-19 pneumonia (COVACTA). Available from: https://clinicaltrials.gov/ct2/show/ NCT04320615. Accessed October 20, 2020.

135. Tocilizumab in COVID-19 pneumonia (TOCIVID-19) (TOCIVID-19). Available from: https://clinicaltrials.gov/ct2/ show/NCT04317092. Accessed October 20, 2020.

136. Tocilizumab for SARS-CoV2 (COVID-19) severe pneumonitis. Available from: https://clinicaltrials.gov/ct2/show/NCT04315480. Accessed October 20, 2020.

137. Anti-il6 treatment of serious COVID-19 disease with threatening respiratory failure (TOCIVID). Available from: https://clinical trials.gov/ct2/show/NCT04322773. Accessed October 20, 2020.

138. CORIMUNO-19-tocilizumab trial-TOCI (CORIMUNO-TOCI) (CORIMUNO-TOC). Available from: https://clinicaltrials.gov/ ct2/show/NCT04331808. Accessed October 20, 2020.

139. Tocilizumab improves significantly clinical outcomes of patients with moderate or severe COVID-19 pneumonia. Available from: https:/pipe linereview.com/index.php/2020042874458/Antibodies/Tocilizumabimproves-significantly-clinical-outcomes-of-patients-with-moderate-orsevere-COVID-19-pneumonia.html. Accessed October 20, 2020.

140. Press release. First randomized study favorable to tocilizumab in Covid-19, in France. Available from: https://www.apmnews.com/ depeche/0/350489/premiere-etude-randomisee-favorable-au-tocilizu mab-dans-le-covid-19\%2C-en-france. Accessed October 20, 2020.

141. Evaluation of the efficacy and safety of sarilumab in hospitalized patients with COVID-19. Available from: https://clinicaltrials. gov/ct2/show/NCT04315298. Accessed October 20, 2020.

142. Erman M, Joseph S. Regeneron, Sanofi arthritis drug may only help critical coronavirus patients: study. Available from: https://www.reu ters.com/article/us-health-coronavirus-regeneron-pharms/regeneronsanofi-to-treat-only-critical-covid-19-patients-with-arthritis-drugidUSKCN2291OD. Accessed October 20, 2020.

143. Cohort multiple randomized controlled trials open-label of immune modulatory drugs and other treatments in COVID-19 patientssarilumab trial-CORIMUNO-19-SARI (CORIMUNO-SARI). Available from: https://clinicaltrials.gov/ct2/show/study/ NCT04324073. Accessed October 20, 2020.

144. Efficacy and safety of siltuximab vs. corticosteroids in hospitalized patients with COVID-19 pneumonia. Available from: https://clin icaltrials.gov/ct2/show/record/NCT04329650?cond=siltuximab + covid+19\&draw=2\&rank=1. Accessed October 20, 2020.

145. Clinical trial of the use of anakinra in cytokine storm syndrome secondary to Covid-19 (ANA-COVID-GEAS) (ANA-COVID-GEAS). Available from: https://clinicaltrials.gov/ct2/show/NCT04443881? term=NCT04443881\&draw=2\&rank=1. Accessed October 20, 2020.

146. Available from: https://www.recoverytrial.net/news/recovery-covid19-phase-3-trial-to-evaluate-regeneron2019s-regn-cov2-investiga tional-antibody-cocktail-in-the-uk. Accessed October 20, 2020.

147. Available from: https://investor.regeneron.com/news-releases/ news-release-details/regenerons-regn-cov2-antibody-cocktailreduced-viral-levels-and. Accessed October 20, 2020.

148. Lo Caputo S, Corso G, Clerici M, et al. Baricitinib: a chance to treat COVID-19? [published online ahead of print, 2020 May 21] J Med Virol. 2020:10.1002/jmv.26033. doi:10.1002/jmv.26033.

149. Recombinant bacterial ACE2 receptors -like enzyme of B38-CAP could be promising COVID-19 infection- and lung injury preventing drug better than recombinant human ACE2 (bacterial ACE2). Available from: https://clinicaltrials.gov/ct2/show/NCT04375046? term $=$ NCT04375046\&draw $=2 \&$ rank=1. Accessed October 20, 2020.

150. Combination of recombinant bacterial ACE2 receptors -like enzyme of B38-CAP and isotretinoin could be promising COVID-19 infection- and lung injury preventing drug better than recombinant human ACE2. Available from: https://clinical trials.gov/ct2/show/NCT04382950?term=NCT04382950\&draw $=$ 2\&rank=1. Accessed November 23, 2020.
151. Evaluation of safety and efficiency of method of exosome inhalation in SARS-CoV-2 associated pneumonia. (COVID-19EXO). Available from: https://clinicaltrials.gov/ct2/show/ NCT04491240? term $=$ NCT04491240\&draw $=2 \&$ rank $=1$.

Accessed October 20, 2020.

152. COVID-19 specific $\mathrm{T}$ cell derived exosomes (CSTC-Exo). Available from: https://clinicaltrials.gov/ct2/show/ NCT04389385? term $=$ NCT04389385\&draw $=2 \&$ rank $=1$. Accessed October 20, 2020

153. Zofin (organicell flow) for patients with COVID-19. Available from: https://clinicaltrials.gov/ct2/show/NCT04384445?term= NCT04384445\&draw=2\&rank=1. Accessed October 20, 2020.

154. A pilot clinical study on inhalation of mesenchymal stem cells exosomes treating severe novel coronavirus pneumonia. Available from: https:/clinicaltrials.gov/ct2/show/NCT04276987?term= NCT04276987\&draw=2\&rank=1. Accessed October 20, 2020.

155. Administration of allogenic UC-MSCs as adjuvant therapy for critically-ill COVID-19 patients. Available from: https://clinical trials.gov/ct2/show/NCT04457609?term $=$ NCT04457609\&draw $=$ 1\&rank=1. Accessed October 20, 2020.

156. Renin-angiotensin system inhibitors and COVID-19 (SARSRAS). Available from: https://clinicaltrials.gov/ct2/show/ NCT04331574?term $=$ NCT04331574\&draw $=2 \&$ rank $=1$.

Accessed October 20, 2020.

157. ACE inhibitors or ARBs discontinuation in context of SARSCoV-2 pandemic (ACORES-2). Available from: https://clinical trials.gov/ct2/show/NCT04329195?term $=$ NCT04329195\&draw $=$ 1\&rank=1. Accessed October 20, 2020.

158. Stopping ACE-inhibitors in COVID-19 (ACEI-COVID). Available from: https://clinicaltrials.gov/ct2/show/ NCT04353596? term $=$ NCT04353596\&draw $=2 \&$ rank $=1$.

Accessed October 20, 2020.

159. TCM differentiation and treatment protocol of COVID-19 (TDATPOC). Available from: https://clinicaltrials.gov/ct2/show/ NCT04306497?term $=$ NCT04306497\&draw $=2 \&$ rank $=1$. Accessed October 20, 2020

160. Treatment and prevention of traditional Chinese medicines (TCMs) on COVID-19 infection. Available from: https://clinical trials.gov/ct2/show/NCT04251871?term $=\mathrm{NCT} 04251871 \&$ draw $=$ 2\&rank=1. Accessed October 20, 2020.

161. Pharmacologic ascorbic acid as an activator of lymphocyte signaling for COVID-19 treatment. Available from: https://clinical trials.gov/ct2/show $/ \mathrm{NCT} 04363216$ ? term $=\mathrm{NCT} 04363216 \&$ draw $=$ 2\&rank=1. Accessed October 20, 2020.

162. Use of ascorbic acid in patients with COVID 19. Available from: https://clinicaltrials.gov/ct2/show/NCT04323514?term= NCT04323514\&draw=2\&rank=1. Accessed October 20, 2020.

163. Vitamin D status and immune-inflammatory status in different UK populations with COVID-19 infection. Available from: https://clinicaltrials.gov/ct2/show/NCT04519034?term = NCT04519034\&draw=2\&rank=1. Accessed October 20, 2020.

164. Anti-inflammatory/antioxidant oral nutrition supplementation in COVID-19 (ONSCOVID19). Available from: https://clinical trials.gov/ct $2 /$ show $/ \mathrm{NCT} 04323228$ ?term $=\mathrm{NCT} 04323228 \& \mathrm{draw}=$ 2\&rank=1.Accessed October 20, 2020.

165. A phase II, controlled clinical study designed to evaluate the effect of artemiC in patients diagnosed with COVID-19. Available from: https://clinicaltrials.gov/ct2/show/ NCT04382040? term $=$ NCT04382040\&draw $=2 \&$ rank $=1$.

Accessed October 20, 2020.

166. Honey \& nigella sativa trial against COVID-19 (HNS-COVIDPK). Available from: https://clinicaltrials.gov/ct2/show/ NCT04347382?term $=$ NCT04347382\&draw $=2 \&$ rank $=1$. Accessed October 20, 2020. 
167. Efficacy of natural honey treatment in patients with novel coronavirus. Available from: https:/clinicaltrials.gov/ct2/show/ NCT04323345?term=NCT04323345\&draw=2\&rank=1.

Accessed October 20, 2020.

168. The role of resistant starch in COVID-19 infection. Available from: https://clinicaltrials.gov/ct2/show/NCT04342689?term= NCT04342689\&draw=2\&rank=1. Accessed October 20, 2020.

169. Gheblawi M, Wang K, Viveiros A, et al. Angiotensin-converting enzyme 2: SARS-CoV-2 receptor and regulator of the reninangiotensin system: celebrating the 20th anniversary of the discovery of ACE2. Circ Res. 2020;126(10):1456-1474. doi:10.1161/CIRCRESAHA.120.317015

170. Hassanpour M, Rezaie J, Nouri M, et al. The role of extracellular vesicles in COVID-19 virus infection [published online ahead of print, 2020 Jun 13]. Infect Genet Evol. 2020;85:104422. doi:10.1016/j.meegid.2020.104422

171. Li X, Corbett AL, Taatizadeh E, et al. Challenges and opportunities in exosome research-perspectives from biology, engineering, and cancer therapy. APL Bioeng. 2019;3(1):011503. doi:10.1063/1.5087122

172. Fu Y, Cheng Y, Wu Y. Understanding SARS-CoV-2-mediated inflammatory responses: from mechanisms to potential therapeutic tools. Virol Sin. 2020;35(3):266. doi:10.1007/s12250-020-00207-4

173. Zhong J, Tang J, Ye C, et al. The immunology of COVID-19: is immune modulation an option for treatment? Lancet Rheumatol. 2020;2(7):e428-e436. doi:10.1016/S2665-9913(20)30120-X

174. Barochiner J, Martínez R. Use of inhibitors of the renin-angiotensin system in hypertensive patients and COVID-19 severity: a systematic review and meta-analysis [published online ahead of print, 2020 Aug 7]. J Clin Pharm Ther. 2020;10.1111/jcpt.13246. doi: $10.1111 /$ jcpt.13246

175. Chen H, Du Q. Potential natural compounds for preventing 2019nCoV infection. Preprints. 2020;2020010358. doi:10.20944/preprints202001.0358.v3

176. Ren JL, Zhang AH, Wang XJ. Traditional Chinese medicine for COVID-19 treatment [published correction appears in pharmacol res. 2020 Mar 25;:104768]. Pharmacol Res. 2020;155:104743. doi:10.1016/j.phrs.2020.104743

177. Wang Y, Zeng X, Zhao Y, et al. The pros and cons of traditional Chinese medicines in the treatment of COVID-19. Pharmacol Res. 2020;157:104873. doi:10.1016/j.phrs.2020.104873

178. Gray PE, Belessis Y. The use of traditional Chinese medicines to treat SARS-CoV-2 may cause more harm than good. Pharmacol Res. 2020;156:104776. doi:10.1016/j.phrs.2020.104776

179. Yoshihara D, Fujiwara N, Suzuki K. Antioxidants: benefits and risks for long-term health. Maturitas. 2010;67(2):103-107. doi:10.1016/j.maturitas.2010.05.001

180. Available from: https://www.webmd.com/drugs/2/drug-15583/ antioxidant-vitamins-oral/details/list-sideeffects. Accessed October 20, 2020.

181. Zahedipour F, Hosseini SA, Sathyapalan T, et al. Potential effects of curcumin in the treatment of COVID-19 infection [published online ahead of print, 2020 May 19]. Phytother Res. 2020:10.1002/ptr.6738. doi:10.1002/ptr.6738.

182. Available from: https:/www.nutraingredients-asia.com/Article/ 2020/04/20/Can-honey-omega-3-resistant-potato-starch-helpfight-COVID-19-Researchers-to-examine-via-clinical-trials. Accessed October 20, 2020.

183. Rogero MM, Leão MC, Santana TM, et al. Potential benefits and risks of omega-3 fatty acids supplementation to patients with COVID-19. Free Radic Biol Med. 2020;156:190-199. doi:10.1016/j.freeradbiomed.2020.07.005

184. Available from: https://www.webmd.com/a-to-z-guides/manukahoney-medicinal-uses\#1. Accessed October 20, 2020.

185. Available from: https://atlasbiomed.com/blog/potato-resistantstarch/. Accessed October 20, 2020.
186. Hossain K, Hossain M, Moni A, et al. Prospects of honey in fighting against COVID-19: pharmacological insights and therapeutic promises. OSF Preprints. 2020. doi:10.31219/osf.io/w3hqu

187. Heiskanen V, Hamblin MR. Photobiomodulation: lasers vs. light emitting diodes? Photochem Photobiol Sci. 2018;17(8):10031017. doi:10.1039/c8pp90049c

188. Solmaz H, Ulgen Y, Gulsoy M. Photobiomodulation of wound healing via visible and infrared laser irradiation. Lasers Med Sci. 2017;32(4):903-910. doi:10.1007/s10103-017-2191-0

189. Gavish L, Houreld NN. Therapeutic efficacy of home-use photobiomodulation devices: a systematic literature review. Photomed Laser Surg. 2019;37(1):4-16. doi:10.1089/photob.2018.4512

190. de Freitas LF, Hamblin MR. Proposed mechanisms of photobiomodulation or low-level light therapy. IEEE J Sel Top Quantum Electron. 2016;22(3):7000417. doi:10.1109/ JSTQE.2016.2561201

191. Amaroli A, Agas D, Lus F, et al. The effects of photobiomodulation of $808 \mathrm{~nm}$ diode laser therapy at higher fluence on the in vitro osteogenic differentiation of bone marrow stromal cell. Front Physiol. 2018;9(123):1-11. doi:10.3389/ fphys.2018.00123

192. Amaroli A, Ferrando S, Hanna R, et al. The photobiomodulation effect of higher-fluence 808-nm laser therapy with a flat-top handpiece on the wound healing of the earthworm Dendrobaena veneta: a brief report. Lasers Med Sci. 2018;33:221-225. doi:10.1007/s10103-016-2132-3

193. Hamblin MR. Mechanisms and applications of the anti-inflammatory effects of photobiomodulation. AIMS Biophys. 2017;4 (3):337-361. doi:10.3934/biophy.2017.3.337

194. Hanna R, Dalvi S, Amaroli A, et al. Effects of photobiomodulation on bone defects grafted with bone substitutes: a systematic review of in vivo animal studies. J Biophotonics. 2020; e202000267:1-41. doi:10.1002/jbio.202000267

195. Hanna R, Agas D, Benedicenti S, et al. A comparative study between the effectiveness of $980 \mathrm{~nm}$ photobiomodulation delivered by hand-piece with Gaussian vs. flat-top profiles on osteoblasts maturation. Front Endocrinol. 2019;10:92. doi:10.3389/ fendo.2019.00092

196. Aimbire F, Albertine R, de Magalhães RG, et al. Effect of LLLT Ga-Al-As $(685 \mathrm{~nm})$ on LPS-induced inflammation of the airway and lung in the rat. Lasers Med Sci. 2005;20(1):11-20. doi:10.1007/s10103-005-0339-9

197. Aimbire F, Lopes-Martins RA, Albertini R, et al. Effect of lowlevel laser therapy on hemorrhagic lesions induced by immune complexes in rat lungs. Photomed Laser Surg. 2007;25(2):112117. doi:10.1089/pho.2006.1041

198. Aimbire F, Ligeiro de Oliveira AP, Albertini R, et al. Low level laser therapy (LLLT) decreases pulmonary microvascular leakage, neutrophil influx and IL-1beta levels in airway and lung from rat subjected to LPS- induced inflammation. Inflammation. 2008;31 (3):189-197. doi:10.1007/s10753-008-9064-4

199. Oliveira MC, Greiffo FR, Rigonato-Oliveira NC, et al. Low level laser therapy reduces acute lung inflammation in a model of pulmonary and extrapulmonary LPS-induced ARDS. J Photochem Photobiol B. 2014;134:57-63. doi:10.1016/j.jphotobiol.2014.03.021

200. Mokmeli S, Vetrici M. Low level laser therapy as a modality to attenuate cytokine storm at multiple levels, enhance recovery, and reduce the use of ventilators in COVID-19. Can J Respir Ther. 2020;56:1-7. doi:10.29390/cjrt-2020-015

201. Mafra de Lima F, Villaverde AB, Salgado MA, et al. Low intensity laser therapy (LILT) in vivo acts on the neutrophils recruitment and chemokines/cytokines levels in a model of acute pulmonary inflammation induced by aerosol of lipopolysaccharide from Escherichia coli in rat. $J$ Photochem Photobiol B. 2010;101:271-278. doi:10.1016/j.jphoto biol.2010.07.012 
202. Brochetti A, Leal MP, Rodrigues R, et al. Photobiomodulation therapy improves both inflammatory and fibrotic parameters in experimental model of lung fibrosis in mice. Lasers Med Sci. 2017;32(8):1825-1834. doi:10.1007/s10103-017-2281-z

203. de Brito AA, da Silveira EC, Rigonato-Liveira NC, et al. Low-level laser therapy attenuates lung inflammation and airway remodeling in a murine model of idiopathic pulmonary fibrosis, relevance to cytokines secretion from lung structural cells. J Photochem Photobiol B. 2020;203:111731. doi:10.1016/j.jphotobiol.2019.111731

204. Lai CC, Shih TP, Ko WC, et al. Severe acute respiratory syndrome coronavirus 2 (SARS-CoV-2) and coronavirus disease-2019 (COVID-19), The epidemic and the challenges. Int $J$ Antimicrob Agents. 2020;55(3):105924. doi:10.1016/j.ijantimicag.2020.105924

205. Miranda da Silva C, Peres Leal M, Brochetti RA, et al. Low level laser therapy reduces the development of lung inflammation induced by formaldehyde exposure. PLoS One. 2015;10(11): e0142816. doi:10.1371/journal.pone.0142816

206. da Cunha Moraes G, Vitoretti LB, de Brito AA, et al. Low-level laser therapy reduces lung inflammation in an experimental model of chronic obstructive pulmonary disease involving $\mathrm{p} 2 \times 7$ receptor. Oxid Med Cell Longev. 2018;2018:6798238. doi:10.1155/ 2018/6798238

207. Enwemeka CS, Bumah VV, Masson-Meyers DS. Light as a potential treatment for pandemic coronavirus infections: a perspective. $J$ Photochem Photobiol B. 2020;207:111891. doi:10.1016/j.jphotobiol.2020.111891

208. Yusupalieva MM, Savtchenko VM. The effectiveness of combined laser therapy for the treatment of the patients presenting with bronchial asthma and concomitant allergic rhinitis. Vopr Kurortol Fizioter Lech Fiz Kult. 2017;94(4):14-18. doi:10.17116/kurort201794414-18

209. Askenova IZ, Burduli NM. Pathogenetic effects of low-intensity laser therapy for chronic obstructive pulmonary disease. Ter Arkh. 2015;88:32-35. doi:10.17116/terarkh201688332-35

210. Sigman SA, Mokmeli S, Monici M, et al. A 57-year-old african american man with severe COVID-19 pneumonia who responded to supportive photobiomodulation therapy (PBMT): first use of PBMT in COVID-19. Am $J$ Case Rep. 2020;21:e926779. doi:10.12659/AJCR.926779

211. Sigman SA, Mokmeli S, Vetrici MA. Adjunct low level laser therapy (LLLT) in a morbidly obese patient with severe COVID-19 pneumonia: a case report. Can J Respir Ther. 2020;56:52-56. doi:10.29390/cjrt-2020-022

212. Giannelli M, Bani D. Appropriate laser wavelengths for photodynamic therapy with methylene blue. Lasers Med Sci. 2018;33 (8):1837-1838. doi:10.1007/s10103-018-2566-x

213. Dias LD, Bagnato VS. An update on clinical photodynamic therapy for fighting respiratory tract infections: a promising tool against COVID-19 and its co-infections. Laser Phys Lett. 2020;17 (8):1-9. doi:10.1088/1612-202X/ab95a9

214. Wainright M, Crossley KB. Photosensitizing agents- circumventing resistance and breaking down biofilms: a review. Int Biodeter Biodegr. 2004;53(2):119-126. doi:10.1016/j. ibiod.2003.11.006

215. Hu XQ, Huang YY, Wang YG. Antimicrobial photodynamic therapy to control clinically relevant biofilm infections. Front Microbiol. 2019;9(1299):1-24. doi:10.3389/fmicb.2018.01299

216. Wiehe A, O'Brien JM, Senge MO. Trends and targets in antiviral phototherapy. Photochem Photobiol Sci. 2019;18(11):2565-2612. doi:10.1039/c9pp00211a

217. Moan J, Berg K, Kvam E, et al. Intracellular localization of photosensitizers. Ciba Found Symp. 1989;146:95-107. doi:10.1002/9780470513842.ch7

218. Park SE. Epidemiology, virology, and clinical features of severe acute respiratory syndrome -coronavirus-2 (SARS-CoV-2; coronavirus disease-19). Clin Exp Pediatr. 2020;63(4):119-124. doi:10.3345/cep.2020.00493
219. Woelfel R, Corman VM, Guggemos W, et al. Virological assessment of hospitalized cases of coronavirus disease 2019. medRxiv. 2020;03:05.20030502. doi:10.1101/2020.03.05.20030502

220. Oh MD, Park WB, Choe PG, et al. Viral load kinetics of MERS coronavirus infection. $N$ Engl J Med. 2016;375(13):1303-1305. doi:10.1056/NEJMc1511695

221. Blanco KC, Inada NM, Carbinatto FM, et al. Treatment of recurrent pharyngotonsillitis by photodynamic therapy. Photodiagnosis Photodyn Ther. 2017;18:138-139. doi:10.1016/j. pdpdt.2017.01.187

222. Sécher T, Alexie Mayor A, Heuzé-Vourc'h N. Inhalation of immuno-therapeutics/- prophylactics to fight respiratory tract infections: an appropriate drug at the right place! Front Immunol. 2019;10(2760):1-6. doi:10.3389/fimmu.2019.02760

223. Moghissi K, Dixon K, Gibbins S. Does PDT have potential in the treatment of COVID-19 patients? Photodiagnosis Photodyn Ther. 2020;31(101889):1-2. doi:10.1016/j.pdpdt.2020.101889

224. Geralde MC, Leite IS, Inada NM, et al. Pneumonia treatment by photodynamic therapy with extracorporeal illumination - an experimental model. Physiol Rep. 2017;5(5):e13190. doi:10.14814/phy2.13190

225. Kassab G, Geralde MC, Inada NM, Achiles AE, Guerra VG, Bagnato VS. Nebulization as a tool for photosensitizer delivery to the respiratory tract. J Biophotonics. 2019;12(4):e201800189. doi:10.1002/jbio.201800189

226. Schikora D, Hepburn J, Plavin SR. Reduction of the viral load by non-invasive photodynamic therapy in early stages of COVID-19 infection. Am J Virol Dis. 2020;2(1):01-05.

227. Jin C, Yu B, Zhang J. Methylene blue photochemical treatment as a reliable SARS-CoV-2 plasma virus inactivation method for blood safety and convalescent plasma therapy for the COVID-19 outbreak. Res Square. 2020;1-15. doi:10.21203/rs.3.rs-17718/v1

228. Keil SD, Bowen R, Marschner S. Inactivation of Middle East respiratory syndrome coronavirus (MERS-CoV) in plasma products using a riboflavin-based and ultraviolet light-based photochemical treatment. Transfusion. 2016;56(12):2948-2952. doi: $10.1111 / \operatorname{trf} .13860$

229. Ruane PH, Edrich R, Gampp D, et al. Photochemical inactivation of selected viruses and bacteria in platelet concentrates using riboflavin and light. Transfusion. 2014;44(6):877. doi:10.1111/ j.1537-2995.2004.03355.x

230. Wagner SJ. Virus inactivation in blood components by photoactive phenothiazine dyes. Transfus Med Rev. 2002;16(1):61-66. doi:10.1053/tmrv.2002.29405

231. Bachmann B, Knüver-Hopf J, Lambrecht B, et al. Target structures for HIV-1 inactivation by methylene blue and light. $J$ Med Virol. 1995;47(2):172-178. doi:10.1002/jmv.1890470211

232. Bumah VV, Masson-Meyers DS, Enwemeka CS. Pulsed 450 nm blue light suppresses MRSA and propionibacterium acnes in planktonic cultures and bacterial biofilms. $J$ Photochem Photobiol B. 2020;202:111702. doi:10.1016/j.jphotob iol.2019.111702

233. Masson-Meyers DS, Bumah VV, Castel C, et al. Pulsed 450 nm blue light significantly inactivates Propionibacterium acnes more than continuous wave blue light. $J$ Photochem Photobiol B. 2020;202:111719. doi:10.1016/j.jphotobiol.2019.111719

234. Hanna R, Dalvi S, Sălăgean T, et al. Phototherapy as a rational antioxidant treatment modality in COVID-19 management; new concept and strategic approach: critical review. Antioxidants. 2020;9(875):1-23. doi:10.3390/antiox9090875

235. Photodynamic therapy for the treatment of COVID-19. Available from: https://clinicaltrials.gov/show/NCT04416113. Accessed October 20, 2020.

236. Photobiomodulation therapy combined with static magnetic field in patients with COVID-19. Available from: https:/clinicalTrials. govshow/NCT04386694. Accessed October 20, 2020. 
237. Lung treatment of lung inflammation in COVID-19. Available from: https://clinicalTrials.govshow/NCT04524715. Accessed October 20, 2020.
238. Photobiomodulation laser therapy for COVID-19 positive patients with pulmonary disease. Available from: https://clinicalTrials.gov show/NCT04391712. Accessed October 20, 2020.

\section{Publish your work in this journal}

The Journal of Inflammation Research is an international, peerreviewed open-access journal that welcomes laboratory and clinical findings on the molecular basis, cell biology and pharmacology of inflammation including original research, reviews, symposium reports, hypothesis formation and commentaries on: acute/chronic inflammation; mediators of inflammation; cellular processes; molecular mechanisms; pharmacology and novel anti-inflammatory drugs; clinical conditions involving inflammation. The manuscript management system is completely online and includes a very quick and fair peerreview system. Visit http://www.dovepress.com/testimonials.php to read real quotes from published authors. 